\title{
A UNIFORMLY CONTROLLABLE AND IMPLICIT SCHEME FOR THE 1-D WAVE EQUATION*
}

\begin{abstract}
ARNAUd MÜNCH ${ }^{1}$
Abstract. This paper studies the exact controllability of a finite dimensional system obtained by discretizing in space and time the linear 1-D wave system with a boundary control at one extreme. It is known that usual schemes obtained with finite difference or finite element methods are not uniformly controllable with respect to the discretization parameters $h$ and $\Delta t$. We introduce an implicit finite difference scheme which differs from the usual centered one by additional terms of order $h^{2}$ and $\Delta t^{2}$. Using a discrete version of Ingham's inequality for nonharmonic Fourier series and spectral properties of the scheme, we show that the associated control can be chosen uniformly bounded in $L^{2}(0, T)$ and in such a way that it converges to the HUM control of the continuous wave, i.e. the minimal $L^{2}$-norm control. The results are illustrated with several numerical experiments.
\end{abstract}

Mathematics Subject Classification. 35L05, 65M60, 93B05.

Received: October 19, 2004. Revised: November 29, 2004.

\section{INTRODUCTION}

In the context of the boundary controllability problem of the 1-D wave equation, the following result is well known [16]: given $T>2$ and $\left(y_{0}, y_{1}\right) \in L^{2}(0,1) \times H^{-1}(0,1)$ there exists a control function $v \in L^{2}(0, T)$ such that the solution of the equation

$$
(\mathcal{S}) \begin{cases}y^{\prime \prime}-y_{x x}=0, & 0<x<1, \quad 0<t<T, \\ y(0, t)=0, y(1, t)=v(t), & 0<t<T, \\ y(x, 0)=y_{0}(x), y^{\prime}(x, 0)=y_{1}(x), & 0<x<1,\end{cases}
$$

satisfies

$$
y(x, T)=y^{\prime}(x, T)=0, \quad \forall 0<x<1 .
$$

We denote by ' the time derivative. This controllability problem has been studied and solved some decades ago. We mention the most successful moments theory (see [24]) and more recently the Hilbert Uniqueness Method (HUM) offering a very general way to solve this kind of problem (see [16]).

\footnotetext{
Keywords and phrases. Exact boundary controllability, wave system, finite difference.

* The author was partially supported by Grants HPRN-CT-2002-00284 of the European program New materials, adaptive systems and their nonlinearities: modelling, control and numerical simulation.

${ }^{1}$ Laboratoire de Mathématiques de Besançon, UMR CNRS 6623, UFR de Sciences et Techniques, Université de Franche-Comté, 16, route de Gray 25030, Besançon cedex, France. arnaud.munch@math.univ-fcomte.fr 


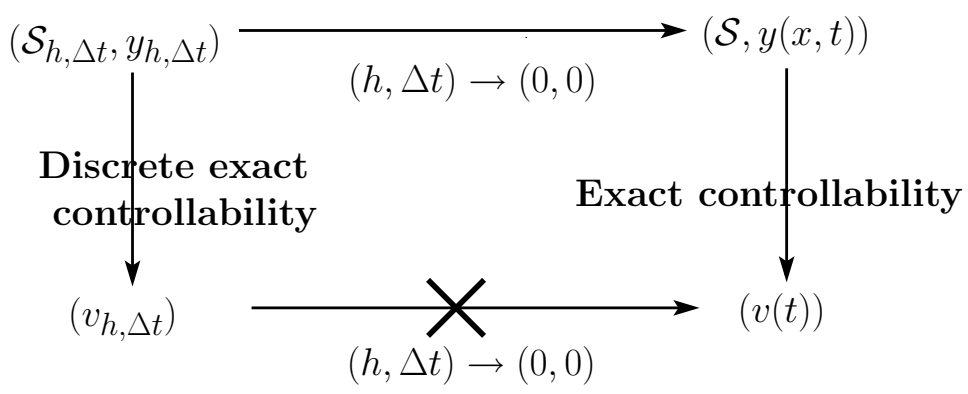

Figure 1. Non commuting diagram associated to the scheme $\left(\mathcal{S}_{h, \Delta t}\right)$ for $\Delta t<h$.

We address in this work the numerical approximation of this problem which is known to be extremely sensitive, since the pioneering work of Glowinski-Li-Lions. In [9], these authors described an algorithm based on the HUM method and on the usual centered finite difference approximation of the wave system (1). This approximation is of the type:

$$
\left(\mathcal{S}_{h, \Delta t}\right)\left\{\begin{array}{l}
\Delta_{\Delta t} y_{h, \Delta t}-\Delta_{h} y_{h, \Delta t}=0 \\
+ \text { Initial conditions and Boundary terms }
\end{array}\right.
$$

where $\Delta_{\Delta t}$ (resp. $\left.\Delta_{h}\right)$ designates the three-point finite difference approximation of the second time (resp. space) derivative operator (see Eq. (15)). Under the stability condition $\Delta t \leq h$, the finite dimensional system $\left(\mathcal{S}_{h, \Delta t}\right)$ is consistent with $(\mathcal{S})$ in the sense that $y_{h, \Delta t}$ converges towards $y$ when $h$ - the mesh size and $\Delta t-$ the time step - tend to zero. However, as observed in [9], the resulting sequence of controls $\left(v_{h, \Delta t}\right)$ obtained with a discrete HUM method has a bad asymptotic behavior as $(h, \Delta t) \rightarrow(0,0)$. Precisely, it is possible to exhibit initial conditions such that $v_{h}$ does not converge towards $v$ but rather diverges exponentially as the mesh size tends to zero [17]. In other words, the commuting property between numerical discretization and exact controllability does not hold for $\left(S_{h, \Delta t}\right)$ (see Fig. 1). This by now well known phenomenon is due to the fact that $\left(S_{h, \Delta t}\right)$ and, in general any discrete dynamics, generates spurious high-frequency oscillations that do not exist at the continuous level [7]. Moreover, the interaction of waves with the grid produces a dispersion phenomenon and the speed of propagation of these high frequency numerical waves may converges to zero when the mesh size tends to zero. These spurious oscillations weakly converge to zero. Consequently, their existence is compatible with the consistency of the numerical scheme. However, when we are dealing with the exact controllability problem, a uniform time for the control of all numerical waves is needed. Since the velocity of propagation of some high frequency numerical waves may tend to zero as the mesh size does, uniform observability and therefore controllability properties of the discrete model may be no longer true for a fixed time $T>0$ independent of $h$.

From a numerical point of view, several techniques have been proposed as possible remedies of the high frequency spurious oscillations. We first mention a Tychonoff regularization procedure successfully implemented in several experiments [8]. Roughly speaking, this method introduces an additional control, tending to zero with the mesh size, acting on the interior of the domain which has the effect to damp the high frequency solution. Using an analogy with numerical pathologies observed in Navier-Stokes problem, mixed finite element methods have been efficiently used, discretizing the velocity and the position in two different finite dimensional spaces [10]. Let us also mention the use of a bi-grid strategy which consists of performing a part of the computation of the algorithm on a coarse grid [9]. These three quite efficient remedies introduced at the beginning of the nineties suffer from a lack of both a theoretical justification and a rigorous convergence analysis. In this respect, we mention the recent work $[3,25]$ based on spectral method.

From the theoretical point of view, many results have been obtained, remedies suggested and justified, but mainly at the semi-discrete level (discretization in space). This fact may be a strong limitation if we know how an additional discretization in time could perturb properties of a scheme. Thus, at the semi-discrete level, it 


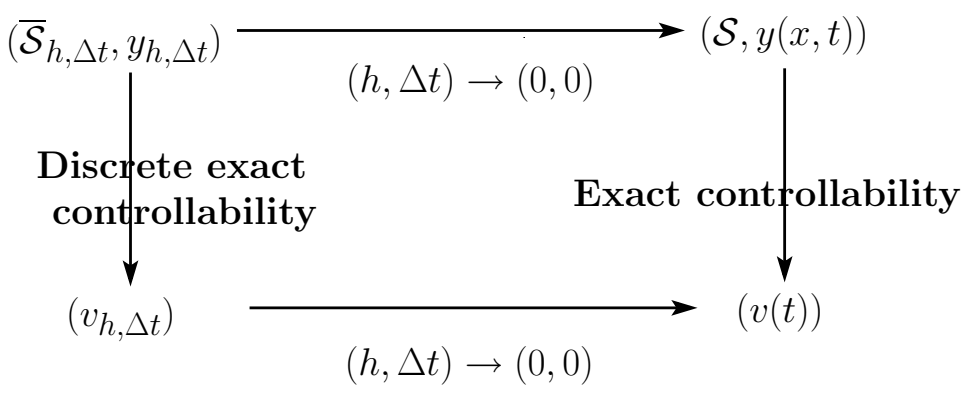

Figure 2. Commuting diagram associated to the scheme $\left(\overline{\mathcal{S}}_{h, \Delta t}\right)$ for $\Delta t<h \sqrt{T / 2}$.

was shown in $[12,26]$ that the filtering of the high frequency modes leads to a uniform observability inequality for the adjoint system. This is equivalent to the uniform controllability of the projection of the solution over the space generated by the remaining eigenmodes. However, in practice, it is not very efficient to compute these projections. In addition, the uniform time $T$ depends strongly on the filtering. Let us also mention the semidiscrete 1-D wave system derived from a mixed finite element method introduced and studied in [4] (see also [2,6]. The authors show the uniform controllability with respect to $h$ of the scheme and obtained the convergence in the $L^{2}$-norm of the semi-discrete control toward the continuous minimal $L^{2}$-norm control. To the knowledge of the author, the only result of convergence obtained at the fully discrete level is from [20]. It is shown that the scheme $\left(\mathcal{S}_{h, \Delta t}\right)$ is uniformly controllable if $\Delta t=h$. This is however a very particular situation because this scheme then provides the exact solution.

In this paper, we prove that the following scheme

$$
\left(\overline{\mathcal{S}}_{h, \Delta t}\right)\left\{\begin{array}{l}
\Delta_{\Delta t} y_{h, \Delta t}+\frac{1}{4}\left(h^{2}-\Delta t^{2}\right) \Delta_{h} \Delta_{\Delta t} y_{h, \Delta t}-\Delta_{h} y_{h, \Delta t}=0 \\
+ \text { Initial conditions and Boundary terms }
\end{array}\right.
$$

produces a discrete uniformly bounded and converging control under the condition $\Delta t<h \sqrt{T / 2}$, and therefore permits to restore the commuting property (see Fig. 2). The scheme $\left(\overline{\mathcal{S}}_{h, \Delta t}\right)$ differs from $\left(\mathcal{S}_{h, \Delta t}\right)$ by additional terms factor of $h^{2}$ and $\Delta t^{2}$ introduced in order to damp out the high frequencies solutions. This scheme is derived from the initial one $\left(\mathcal{S}_{h, \Delta t}\right)$ by introducing two parameters, acting on the consistency and on the uniform controllability respectively. The determination of the admissible parameters, using a discrete version of an Ingham inequality for nonharmonic series, then leads to $\left(\overline{\mathcal{S}}_{h, \Delta t}\right)$.

The rest of the paper is organized as follows. The first section briefly recalls some controllability results for the wave equation (1). In the second section, the fully discrete model is introduced and the main results of existence, characterization and convergence of the discrete control are presented. The third section studies the corresponding homogeneous system. The main result is a uniform observability inequality which is fundamental for the study. In the fourth section, the controllability problem for the discrete system is addressed and a uniformly bounded sequence of controls is given. Next, the convergence of the discrete controls to the HUM control of the continuous equation is proved. The fifth section is devoted to the presentation of some numerical results which are in full agreement with the theoretical study. Results of this paper were partially announced in $[18]$.

\section{The CONTINuOUs PROBLEM: RESUlts And NOTATIONS}

In this section, we recall briefly the controllability property of the wave system (1). For further details, we refer for instance to [16]. 
Theorem 1.1. Given any $T>2$ and $\left(y_{0}, y_{1}\right) \in L^{2}(0,1) \times H^{-1}(0,1)$ there exists a control function $v \in L^{2}(0, T)$ such that the solution $y$ of (1) satisfies (2).

According to the Hilbert Uniqueness Method (HUM), the control of minimal $L^{2}$-norm can be obtained by minimizing the functional $\mathcal{J}: H_{0}^{1}(0,1) \times L^{2}(0,1) \rightarrow \mathbb{R}$ defined as follows:

$$
\mathcal{J}\left(w_{0}, w_{1}\right)=\frac{1}{2} \int_{0}^{T}\left(w_{x}(1, t)\right)^{2} \mathrm{~d} t+\int_{\Omega} y_{0}(x) w^{\prime}(x, 0) \mathrm{d} x-\left\langle y_{1}, w(\cdot, 0)\right\rangle_{H^{-1}, H_{0}^{1}},
$$

where $\langle\cdot, \cdot\rangle_{H^{-1}, H_{0}^{1}}$ denotes the duality product between $H^{-1}(0,1)$ and $H_{0}^{1}(0,1)$ and $w$ the solution of the adjoint backward homogeneous equation

$$
\begin{cases}w^{\prime \prime}-w_{x x}=0, & 0<x<1, \quad 0<t<T \\ w(0, t)=w(1, t)=0, & 0<t<T \\ w(x, T)=w_{0}(x), w^{\prime}(x, T)=w_{1}(x), & 0<x<1\end{cases}
$$

Then, the following result holds.

Theorem 1.2. Given any $T>2$ and $\left(y_{0}, y_{1}\right) \in L^{2}(0,1) \times H^{-1}(0,1)$ the functional $\mathcal{J}$ has a unique minimizer $\left(\hat{w}_{0}, \hat{w}_{1}\right) \in H_{0}^{1}(0,1) \times L^{2}(0,1)$. If $\hat{\omega}$ is the corresponding solution of $(6)$ with initial data $\left(\hat{w}_{0}, \hat{w}_{1}\right)$ then $v(t)=$ $\hat{w}_{x}(1, t)$ is the control of (1) with minimal $L^{2}$-norm.

Remark 1.3. The control $v$ from Theorem 1.2 is usually referred as the HUM control. It may be characterized by the following property:

(1) $v$ is a control of (1) or equivalently,

$$
\int_{0}^{T} v(t) w_{x}(1, t) \mathrm{d} t=\left\langle y_{1}, w(., 0)\right\rangle_{H^{-1}, H_{0}^{1}}-\int_{0}^{1} y_{0}(x) w^{\prime}(x, 0) \mathrm{d} x
$$

for any $\left(w_{0}, w_{1}\right) \in H_{0}^{1}(0,1) \times L^{2}(0,1), w$ being the solution of the adjoint system (6).

(2) There exists $\left(\hat{w}_{0}, \hat{w}_{1}\right) \in H_{0}^{1}(0,1) \times L^{2}(0,1)$ such that $v(t)=\hat{w}_{x}(t, 1)$, where $\hat{w}$ is solution of the adjoint system (6) with initial data $\left(\hat{w}_{0}, \hat{w}_{1}\right)$.

This controllability property is equivalent to the existence of a constant $C(T)$ such that the observability inequality

$$
E(t) \leq C(T) \int_{0}^{T}\left|u_{x}(1, t)\right|^{2} \mathrm{~d} t
$$

where $E$ is the conservative energy holds, i.e. $E(t)=E(0), \forall t \in[0, T]$,

$$
E(t) \equiv \frac{1}{2} \int_{0}^{1}\left[\left|u^{\prime}(x, t)\right|^{2}+\left|u_{x}(x, t)\right|^{2}\right] \mathrm{d} x, \quad \forall 0 \leq t \leq T,
$$

associated to $u$ solution of the system

$$
(\mathcal{A}) \begin{cases}u^{\prime \prime}-u_{x x}=0, & 0<x<1, \quad 0<t<T, \\ u(0, t)=u(1, t)=0, & 0<t<T, \\ u(x, 0)=u_{0}(x), u^{\prime}(x, 0)=u_{1}(x), & 0<x<1 .\end{cases}
$$

Let us finally remark that the eigenvalues of system (1) are given by $\lambda_{k}=(k \pi)^{2}, k>0$ and the corresponding eigenfunctions $\left(\phi_{k}\right)_{k>0}$ are given by $\phi_{k}(x)=\sin \left(\sqrt{\lambda_{k}} x\right)$. Finally, we assume that any initial data $\left(y_{0}, y_{1}\right)$ of (1) may be expanded in Fourier series as follows:

$$
y_{0}=\sum_{k>0} \bar{a}_{k} \phi_{k}, \quad y_{1}=\sum_{k>0} \bar{b}_{k} \phi_{k}
$$


such that the solution $y$ of (1) can be written

$$
y(., t)=\sum_{k>0}\left[\bar{a}_{k} \cos \left(\sqrt{\lambda_{k}} t\right)+\frac{\bar{b}_{k}}{\sqrt{\lambda_{k}}} \sin \left(\sqrt{\lambda_{k}} t\right)\right] \phi_{k} .
$$

\section{A fully Discrete finite difference model - Discrete Controllability}

\subsection{Presentation of a discrete scheme associated to the wave equation}

We introduce a finite difference approximation in space and time of the wave equation (1). Let us consider $J \in \mathbb{N}, h=1 /(J+1)$ and a uniform grid of the space interval $(0,1)$ given by $0=x_{0}<x_{1}<\ldots<x_{J}<x_{J+1}=1$, with $x_{j}=j h, j=0, \ldots, J+1$. Let us also consider $N \in \mathbb{N}, \Delta t=T / N$ and a uniform grid of the time interval $(0, T)$ given by $0=t_{0}<t_{1}<\ldots<t_{N}=1$, with $t_{n}=n \Delta t, n=0, \ldots, N$. $h$ and $\Delta t$ are the space and time step respectively. Let us denote by $y_{j}^{n}$ the approximation of $y$ at the node $x_{j}$ and at time $t_{n}$ :

$$
y_{j}^{n} \approx y\left(x_{j}, t_{n}\right), \quad 0 \leq j \leq J+1,0 \leq n \leq N
$$

We then associate to the continuous wave equation (1) the following discrete scheme:

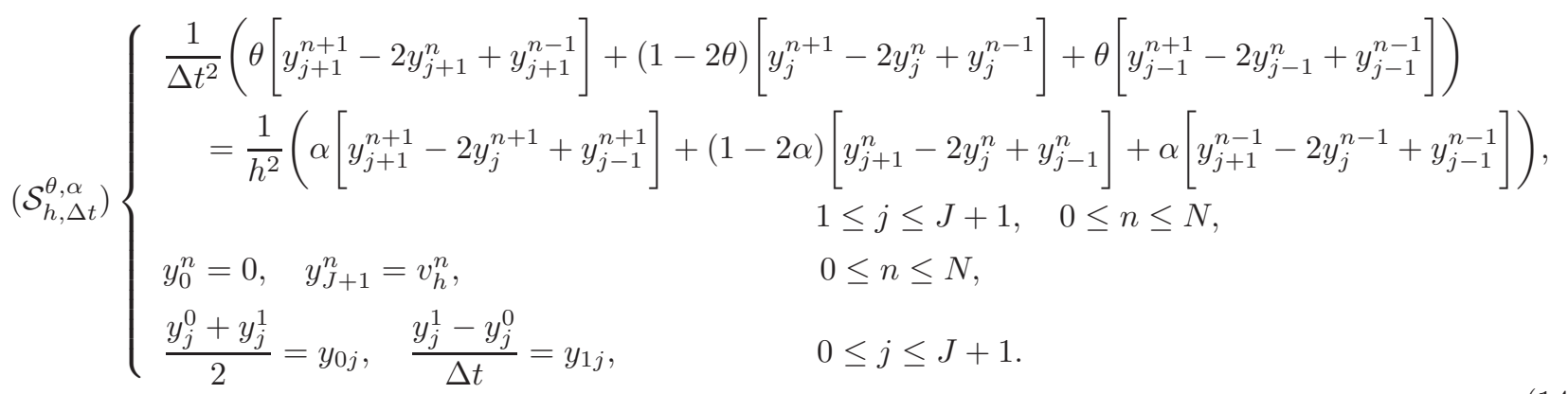

Defining for $j=1, \ldots, J$ and $n=0, \ldots, N$ the following operators:

$$
\Delta_{h} y_{j}^{n}=\frac{y_{j+1}^{n}-2 y_{j}^{n}+y_{j-1}^{n}}{h^{2}}, \quad \Delta_{\Delta t} y_{j}^{n}=\frac{y_{j}^{n+1}-2 y_{j}^{n}+y_{j}^{n-1}}{\Delta t^{2}},
$$

the first equation of $\left(\mathcal{S}_{h, \Delta t}^{\theta, \alpha}\right)$ can be simply rewritten as follows:

$$
\left(1+\theta h^{2} \Delta_{h}\right) \Delta_{\Delta t} y_{j}^{n}=\left(1+\alpha \Delta t^{2} \Delta_{\Delta t}\right) \Delta_{h} y_{j}^{n}, \quad 1 \leq j \leq J, \quad 0 \leq n \leq N
$$

Then, according to the property $\Delta_{\Delta t}\left(\Delta_{h} y_{j}^{n}\right)=\Delta_{h}\left(\Delta_{\Delta_{t}} y_{j}^{n}\right), j=0, \ldots, J+1, n=0, \ldots, N$, the scheme $\left(\mathcal{S}_{h, \Delta t}^{\theta, \alpha}\right)$ is equivalent to

where

$$
\left(\mathcal{S}_{h, \Delta t}^{\theta_{\nu}, 0}\right) \begin{cases}\left(1+\theta_{\nu} h^{2} \Delta_{h}\right) \Delta_{\Delta t} y_{j}^{n}=\Delta_{h} y_{j}^{n}, & 1 \leq j \leq J, \quad 0 \leq n \leq N \\ y_{0}^{n}=0, y_{J+1}^{n}=v_{h}^{n}, & 0 \leq n \leq N \\ \frac{y_{j}^{0}+y_{j}^{1}}{2}=y_{0 j}, \quad \frac{y_{j}^{1}-y_{j}^{0}}{\Delta t}=y_{1 j}, & 0 \leq j \leq J+1,\end{cases}
$$

$$
\theta_{\nu} \equiv \theta-\alpha \nu^{2} ; \quad \nu \equiv \Delta t / h
$$

We assume that the parameters $\theta, \alpha$ are positives and independent of $h$ and that the ratio $\nu$ is of order one, i.e. $\Delta t$ and $h$ are of same order (on the contrary, see Rem. 3.15 below). 


\section{Remark 2.1.}

- $\theta=\alpha=0$ leads to $\theta_{\nu}=0$. Therefore, the scheme $\left(\mathcal{S}_{h, \Delta t}^{0,0}\right)$ corresponds to the usual centered finite difference scheme.

- $\left(\mathcal{S}_{h, \Delta t}^{0, \alpha}\right)$ corresponds to a Newmark scheme (see [23]) introduced in general in order to improve the stability of the scheme.

Let us rewrite the scheme $\left(\mathcal{S}_{h, \Delta t}^{\theta_{\nu}, 0}\right)$ in an equivalent vectorial form: we denote by $K \in \mathcal{M}_{J \times J}(\mathbb{R})$ the tri-diagonal matrix associated to the approximation of the Laplacean:

$$
K=\left(\begin{array}{cccccc}
2 & -1 & & & & \\
-1 & 2 & -1 & & (0) & \\
& -1 & \ddots & \ddots & & \\
& & \ddots & \ddots & -1 & \\
& (0) & & -1 & 2 & -1 \\
& & & & -1 & 2
\end{array}\right)_{J \times J}
$$

and by $M_{0}^{\theta_{\nu}} \in \mathcal{M}_{J \times J}(\mathbb{R})$ the mass matrix:

$$
M_{0}^{\theta_{\nu}}=I-\theta_{\nu} K
$$

If we denote by $\boldsymbol{Y}_{\boldsymbol{h}}^{\boldsymbol{n}}$ the vector $\left(y_{1}^{n}, y_{2}^{n}, \ldots, y_{J}^{n}\right)^{T}, n=0, \ldots, N$, then $\left(\mathcal{S}_{h, \Delta t}^{\theta_{\nu}, 0}\right)$ takes the following vectorial form:

$$
\left\{\begin{array}{c}
M_{0}^{\theta_{\nu}}\left(\boldsymbol{Y}_{\boldsymbol{h}}^{\boldsymbol{n}+\mathbf{1}}-2 \boldsymbol{Y}_{\boldsymbol{h}}^{\boldsymbol{n}}+\boldsymbol{Y}_{\boldsymbol{h}}^{\boldsymbol{n}-\mathbf{1}}\right)+\nu^{2} K \boldsymbol{Y}_{\boldsymbol{h}}^{\boldsymbol{n}}=\boldsymbol{F}_{\boldsymbol{h}}^{\boldsymbol{n}}, \quad 0 \leq n \leq N \\
\frac{\boldsymbol{Y}_{\boldsymbol{h}}^{\mathbf{0}}+\boldsymbol{Y}_{\boldsymbol{h}}^{\mathbf{1}}}{2}=\boldsymbol{y}_{\mathbf{0} \boldsymbol{h}}, \frac{\boldsymbol{Y}_{\boldsymbol{h}}^{\mathbf{1}}-\boldsymbol{Y}_{\boldsymbol{h}}^{\mathbf{0}}}{\Delta t}=\boldsymbol{y}_{\mathbf{1} \boldsymbol{h}},
\end{array}\right.
$$

where $\boldsymbol{F}_{\boldsymbol{h}}^{\boldsymbol{n}}=\left(f_{1}^{n}, \ldots, f_{J-1}^{n}, f_{J}^{n}\right)$ with $f_{j}^{n}=0, j=1 \ldots J-1$, and

$$
f_{J}^{n}=-\theta_{\nu}\left(v_{h}^{n+1}-2 v_{h}^{n}+v_{h}^{n-1}\right)+\nu^{2} v_{h}^{n}, \quad 0 \leq n \leq N,
$$

taking into account that $y_{J+1}^{n}=v_{h}^{n}$ and $y_{0}^{n}=0$, for $n=0 \ldots N$.

Definition 2.2. Let $\boldsymbol{U}, \boldsymbol{V} \in \mathbb{R}^{J}$. We define the following scalar products:

$$
\langle\boldsymbol{U}, \boldsymbol{V}\rangle=\sum_{j=1}^{J} U_{j} V_{j}, \quad(\boldsymbol{U}, \boldsymbol{V})=h\langle\boldsymbol{U}, \boldsymbol{V}\rangle
$$

Definition 2.3. Let $(\boldsymbol{U}, \boldsymbol{V}) \in \mathbb{R}^{2 J}$ and $(\tilde{\boldsymbol{U}}, \tilde{\boldsymbol{V}}) \in \mathbb{R}^{2 J}$. We define the symmetric products on $\mathbb{R}^{2 J}$ :

$$
\begin{aligned}
& ((\boldsymbol{U}, \boldsymbol{V}),(\tilde{\boldsymbol{U}}, \tilde{\boldsymbol{V}}))_{0} \equiv\left(K_{h} \tilde{\boldsymbol{U}}, \boldsymbol{U}\right)+\left(M_{0}^{\theta_{\nu}} \tilde{\boldsymbol{V}}, \boldsymbol{V}\right), \\
& ((\boldsymbol{U}, \boldsymbol{V}),(\tilde{\boldsymbol{U}}, \tilde{\boldsymbol{V}}))_{1} \equiv\left(K_{h} \tilde{\boldsymbol{U}}, \boldsymbol{U}\right)+\left(M_{1}^{\theta_{\nu}} \tilde{\boldsymbol{V}}, \boldsymbol{V}\right),
\end{aligned}
$$

where

$$
M_{1}^{\theta_{\nu}}=M_{0}^{\theta_{\nu}}-\frac{\nu^{2}}{4} K, \quad K_{h}=\frac{K}{h^{2}} .
$$

Finally, we introduce the following subset of $\mathbb{R}^{+} \times \mathbb{R}^{+} \times \mathbb{R}_{\star}^{+}$:

\section{Definition 2.4.}

$$
\mathcal{S}=\left\{(\theta, \alpha, \nu) \in \mathbb{R}^{+} \times \mathbb{R}^{+} \times \mathbb{R}_{\star}^{+}, \cos ^{2}\left(\frac{\pi h}{2}\right)\left(\nu^{2}(1-4 \alpha)+4 \theta\right) \leq 1, \forall h>0\right\} .
$$




\subsection{Discrete controllability - notations and results}

Our main goal is to study the controllability property associated to the discrete case: given $T$ large enough independent of $h$ and $\Delta t$ and $\left(\boldsymbol{y}_{\mathbf{0}}, \boldsymbol{y}_{\mathbf{1} \boldsymbol{h}}\right) \in \mathbb{R}^{2 J}$, does there exist a control function $\left(v_{h}^{n}\right)_{n}, n=0 \ldots N$, such that the solution $\boldsymbol{Y}_{\boldsymbol{h}}^{\boldsymbol{n}}$ of (21) satisfies

$$
\boldsymbol{Y}_{h}^{N}=\mathbf{0}, \quad \frac{\boldsymbol{Y}_{h}^{N}-\boldsymbol{Y}_{h}^{N-1}}{\Delta t}=\mathbf{0},
$$

and therefore $\boldsymbol{Y}_{\boldsymbol{h}}^{\boldsymbol{N}}=\boldsymbol{Y}_{\boldsymbol{h}}^{\boldsymbol{N - 1}}=\mathbf{0}$ ? If this holds for any $\left(\boldsymbol{y}_{\mathbf{0}}, \boldsymbol{y}_{\mathbf{1} \boldsymbol{h}}\right) \in \mathbb{R}^{2 J}$, we say that the system (21) is exactly controllable.

This finite dimensional controllability problem we have just addressed has a positive answer and a sequence of discrete controls $\left(v_{h}\right)_{h>0}$ may be found. Let us now describe how this sequence may be constructed such that the convergence to the HUM control of the continuous problem is ensured. We introduce the Fourier expansion of the initial data $\left(\boldsymbol{y}_{\mathbf{0} \boldsymbol{h}}, \boldsymbol{y}_{\mathbf{1} \boldsymbol{h}}\right)$ in (21). Remark that the eigenvalues $\lambda_{k, h}^{\theta, \alpha}, k=1, \ldots, J$ of system (21) are given, for all $(\theta, \alpha, \nu) \in \mathcal{S}$, by:

$$
\lambda_{k, h}^{\theta, \alpha}=\left[\frac{2}{\Delta t} \arcsin \left(\frac{\nu \sin (k \pi h / 2)}{\sqrt{1-4\left(\theta-\alpha \nu^{2}\right) \sin ^{2}(k \pi h / 2)}}\right)\right]^{2}, \quad \forall k=1, \ldots, J,
$$

such that $0<\lambda_{1, h}^{\theta, \alpha}<\lambda_{2, h}^{\theta, \alpha}<\ldots<\lambda_{J, h}^{\theta, \alpha}$ while the corresponding eigenfunctions $\left(\phi_{\boldsymbol{k}, \boldsymbol{h}}\right)_{(1 \leq k \leq J)}$ are given by $\boldsymbol{\phi}_{\boldsymbol{k}, \boldsymbol{h}}=\left(\phi_{k, j}\right)_{(1 \leq j \leq J)}$, with $\phi_{k, j}=\sin (k \pi j h)$. We then assume that any initial data $\left(\boldsymbol{y}_{\mathbf{0} \boldsymbol{h}}, \boldsymbol{y}_{\mathbf{1} \boldsymbol{h}}\right)$ may be expanded in Fourier series as follows:

$$
\begin{aligned}
& \boldsymbol{y}_{\mathbf{0} \boldsymbol{h}}=\frac{1}{2} \sum_{k=1}^{J}\left[\bar{a}_{k, h}\left(1+\cos \left(\sqrt{\lambda_{k, h}^{\theta, \alpha}} \Delta t\right)\right)+\frac{\bar{b}_{k, h}}{\sqrt{\lambda_{k, h}^{\theta, \alpha}}} \sin \left(\sqrt{\lambda_{k}^{\theta, \alpha}} \Delta t\right)\right] \boldsymbol{\phi}_{\boldsymbol{k}, \boldsymbol{h}}, \\
& \boldsymbol{y}_{\mathbf{1} \boldsymbol{h}}=\sum_{k=1}^{J}\left[\bar{a}_{k, h} \frac{\cos \left(\sqrt{\lambda_{k, h}^{\theta, \alpha}} \Delta t\right)-1}{\Delta t}+\frac{\bar{b}_{k, h}}{\sqrt{\lambda_{k, h}^{\theta, \alpha}}} \frac{\sin \left(\sqrt{\lambda_{k, h}^{\theta, \alpha}} \Delta t\right)}{\Delta t}\right] \boldsymbol{\phi}_{\boldsymbol{k}, \boldsymbol{h}} .
\end{aligned}
$$

Therefore, $\boldsymbol{Y}_{\boldsymbol{h}}^{\boldsymbol{n}}$ takes the following form:

$$
\boldsymbol{Y}_{\boldsymbol{h}}^{\boldsymbol{n}}=\sum_{k=1}^{J}\left[\bar{a}_{k, h} \cos \left(\sqrt{\lambda_{k, h}^{\theta, \alpha}} n \Delta t\right)+\frac{\bar{b}_{k, h}}{\sqrt{\lambda_{k, h}^{\theta, \alpha}}} \sin \left(\sqrt{\lambda_{k, h}^{\theta, \alpha}} n \Delta t\right)\right] \boldsymbol{\phi}_{\boldsymbol{k}, \boldsymbol{h}}
$$

In the sequel, $\left(\bar{a}_{k, h}\right)_{(k>0)}$ and $\left(\bar{b}_{k, h}\right)_{(k>0)}$ will denote the sequence of the Fourier coefficients extended by zero, i.e. we assume $\bar{a}_{k, h}=\bar{b}_{k, h}=0$ when $k>J$.

Let us also consider a cut-off function $\rho \in C_{c}^{\infty}[0, T]$ such that $\rho(t)=1$ for $t \in[\epsilon, T-\epsilon]$, for any $\epsilon>0$. Taking $\epsilon=2 \Delta t$, we approximate this function by $\rho^{n}=\rho(n \Delta t)=1$ for $n \in[2, N-2]$ and $\rho^{n}=0$ otherwise. We then define the discrete version $\mathcal{J}_{h}: \mathbb{R}^{2 J} \rightarrow \mathbb{R}$ of the functional $\mathcal{J}$ defined in (5) as follows:

$$
\begin{aligned}
& \mathcal{J}_{h}\left(\boldsymbol{w}_{\mathbf{0} \boldsymbol{h}}, \boldsymbol{w}_{\mathbf{1} \boldsymbol{h}}\right)=\frac{\Delta t}{2} \sum_{n=0}^{N-1}\left[\left(\frac{w_{J}^{n}}{h}\right)^{2}+\rho^{n} \theta_{\nu}\left(\frac{w_{J}^{n+1}-w_{J}^{n}}{\Delta t}\right)^{2}\right] \\
& -\left(\left(-K_{h}^{-1} M_{0}^{\theta_{\nu}} \boldsymbol{y}_{\mathbf{1} \boldsymbol{h}},\left(M_{1}^{\theta_{\nu}}\right)^{-1} M_{0}^{\theta_{\nu}} \boldsymbol{y}_{\mathbf{0}}\right),\left(\frac{\boldsymbol{W}_{\boldsymbol{h}}^{\mathbf{0}}+\boldsymbol{W}_{\boldsymbol{h}}^{\mathbf{1}}}{2}, \frac{\boldsymbol{W}_{\boldsymbol{h}}^{\mathbf{1}}-\boldsymbol{W}_{\boldsymbol{h}}^{\mathbf{0}}}{\Delta t}\right)\right)_{1},
\end{aligned}
$$


where $\boldsymbol{W}_{\boldsymbol{h}}^{\boldsymbol{n}}$ is the solution of the following adjoint homogeneous system:

$$
\left\{\begin{array}{l}
M_{0}^{\theta_{\nu}}\left(\boldsymbol{W}_{\boldsymbol{h}}^{\boldsymbol{n}+\mathbf{1}}-2 \boldsymbol{W}_{\boldsymbol{h}}^{\boldsymbol{n}}+\boldsymbol{W}_{\boldsymbol{h}}^{\boldsymbol{n}-\mathbf{1}}\right)+\nu^{2} K \boldsymbol{W}_{\boldsymbol{h}}^{\boldsymbol{n}}=0, \quad 0 \leq n \leq N \\
\frac{\boldsymbol{W}_{\boldsymbol{h}}^{\boldsymbol{N}-\mathbf{1}}+\boldsymbol{W}_{\boldsymbol{h}}^{\boldsymbol{N}}}{2}=\boldsymbol{w}_{\mathbf{0 h}}, \frac{\boldsymbol{W}_{\boldsymbol{h}}^{\boldsymbol{N}}-\boldsymbol{W}_{\boldsymbol{h}}^{\boldsymbol{N}-\mathbf{1}}}{\Delta t}=\boldsymbol{w}_{\mathbf{1} \boldsymbol{h}}
\end{array}\right.
$$

Let us then introduce the following definition:

Definition 2.5. We define $\mathcal{C}=\mathcal{C}_{1} \cup \mathcal{C}_{2}$ with

$$
\begin{aligned}
& \mathcal{C}_{1}=\left\{(\theta, \alpha, \nu) \in \mathbb{R}^{+} \times \mathbb{R}^{+} \times \mathbb{R}_{\star}^{+}, \theta=\alpha=1 / 4\right\}, \\
& \mathcal{C}_{\mathbf{2}}=\left\{(\theta, \alpha, \nu) \in \mathbb{R}^{+} \times \mathbb{R}^{+} \times \mathbb{R}_{\star}^{+}, \nu=\sqrt{\frac{1-4 \theta}{1-4 \alpha}},(1-4 \alpha)(1-4 \theta)>0\right\} .
\end{aligned}
$$

Remark 2.6. We have $\mathcal{C} \subset \mathcal{S}$. Actually, $\alpha, \theta$ and $\nu$ being independent of $h$, it is easy to see that

$$
\mathcal{C}=\left\{(\theta, \alpha, \nu) \in \mathbb{R}^{+} \times \mathbb{R}^{+} \times \mathbb{R}_{\star}^{+}, \lim _{h \rightarrow 0} \cos ^{2}(\pi h / 2)\left(\nu^{2}(1-4 \alpha)+4 \theta\right)=1\right\}
$$

The relation between the controllability of (21) and the functional $\mathcal{J}_{h}$ is given by the following theorem which is a discrete version of Theorem 1.2.

Theorem 2.7. Let $(\theta, \alpha, \nu) \in \mathcal{C}$. Given any $T>2 \max \left(1, \nu^{2}\right)$ and $\left(\boldsymbol{y}_{\mathbf{0} \boldsymbol{h}}, \boldsymbol{y}_{\mathbf{1} \boldsymbol{h}}\right) \in \mathbb{R}^{2 J}$, the functional $\mathcal{J}_{h}$ defined by (31) has a unique minimizer $\left(\hat{\boldsymbol{w}}_{\mathbf{0 h}}, \hat{\boldsymbol{w}}_{\mathbf{1} \boldsymbol{h}}\right) \in \mathbb{R}^{2 J}$. Let $v_{h}=\left(v_{h}^{n}\right)_{n}$ defined as follows:

$$
\left\{\begin{array}{l}
v_{h}^{n}-\theta_{\nu} h^{2} \frac{v_{h}^{n+1}-2 v_{h}^{n}+v_{h}^{n-1}}{\Delta t^{2}}=\frac{\hat{W}_{J}^{n}}{h}-\theta_{\nu} h \frac{\rho^{n} \frac{\hat{W}_{J}^{n+1}-\hat{W}_{J}^{n}}{\Delta t}-\rho^{n-1} \frac{\hat{W}_{J}^{n}-\hat{W}_{J}^{n-1}}{\Delta t}}{\Delta t}, \quad 0 \leq n \leq N, \\
\theta_{\nu}\left(v_{h}^{1}-v_{h}^{0}\right)=0, \quad \theta_{\nu}\left(v_{h}^{N}-v_{h}^{N-1}\right)=0,
\end{array}\right.
$$

where $\hat{\boldsymbol{W}}_{\boldsymbol{h}}^{\boldsymbol{n}}$ is the solution of (32) with initial data $\left(\hat{\boldsymbol{w}}_{\mathbf{0} \boldsymbol{h}}, \hat{\boldsymbol{w}}_{\mathbf{1} \boldsymbol{h}}\right)$. Then, $v_{h}=\left(v_{h}^{n}\right)_{n}$ is a control for (21).

The proof of Theorem 2.7 will be given in Section 4. We now define the extensions $Q$ and $P$ of $v_{h}$ on $[0, T]$ :

$$
\left\{\begin{array}{l}
Q\left(v_{h}\right)=\left\{\begin{array}{l}
\text { the piecewise constant function in each interval }\left[t_{n}, t_{n+1}[\text { such that }\right. \\
Q\left(v_{h}\right)\left(t_{n}\right)=v_{h}^{n}, \quad 0 \leq n \leq N-1 .
\end{array}\right. \\
P\left(v_{h}\right)=\left\{\begin{array}{l}
\text { the continuous function linear in each interval }\left[t_{n}, t_{n+1}\right] \text { such that } \\
P\left(v_{h}\right)\left(t_{n}\right)=v_{h}^{n}, \quad 0 \leq n \leq N-1 .
\end{array}\right.
\end{array}\right.
$$

One of the main results of this paper is the following convergence theorem.

Theorem 2.8. Let $(\theta, \alpha, \nu) \in \mathcal{C}$ and $T>2 \max \left(1, \nu^{2}\right)$. Let $\left(\boldsymbol{Y}_{\mathbf{O} \boldsymbol{h}}, \boldsymbol{Y}_{\mathbf{1} \boldsymbol{h}}\right)$ be a sequence of discretizations of the continuous initial data $\left(y_{0}, y_{1}\right)$. Assume that $\left(a_{k, h}, b_{k, h}\right)_{k}$, the Fourier coefficients of the discrete initial data verify

$$
\left(a_{k, h}\right)_{k} \rightarrow\left(a_{k}\right)_{k},\left(\frac{b_{k, h}}{\sqrt{\lambda_{k, h}^{\theta, \alpha}}}\right)_{k} \rightarrow\left(\frac{b_{k}}{k \pi}\right)_{k} \text { in } l^{2} \text { when } h \rightarrow 0,
$$

where $\left(a_{k}, b_{k}\right)$ are the Fourier coefficients of the continuous initial data. Let $\left(v_{h}\right)_{h}$ be the sequence of controls given by Theorem 2.7. Then $\left(Q\left(v_{h}\right)\right)_{h}$ and $\left(h P\left(v_{h}\right)^{\prime}\right)_{h}$ are uniformly bounded in $L^{2}(0, T),\left(h^{2} P\left(v_{h}\right)^{\prime}\right)_{h}$ 
is uniformly bounded in $L^{\infty}(0, T)$ and there exist a subsequence (denoted in the same way) and $v \in L^{2}(0, T)$ such that

$$
\begin{aligned}
& Q\left(v_{h}\right) \rightarrow v \in L^{2}([0, T]) \text { when } h \rightarrow 0, \\
& h P\left(v_{h}\right)^{\prime} \rightarrow 0 \in L^{2}([0, T]) \text { when } h \rightarrow 0, \\
& h^{2} P\left(v_{h}\right)^{\prime} \rightarrow 0 \in L^{\infty}([0, T]) \text { when } h \rightarrow 0 .
\end{aligned}
$$

Moreover, the limit $v$ is the HUM control of the continuous system (1).

If the convergence in (37) is strong, then the above convergences are strong too.

The proof of Theorem 2.8 will be given in Section 5 .

Remark 2.9. $\Delta t$ and $h$ being of same order, the limit $h \rightarrow 0$ in Theorem 2.8 implicitly implies that $\Delta t \rightarrow 0$.

\section{The homogeneous system}

As recalled in Section 2, the controllability property stated in Theorem 1.1 is equivalent to an observability inequality for the homogeneous system. In this section, we prove that a discrete version of such inequality holds for the following homogeneous system:

$$
\left(\mathcal{A}_{h, \Delta t}^{\theta, \alpha}\right) \begin{cases}\left(1+\theta_{\nu} h^{2} \Delta_{h}\right) \Delta_{\Delta t} u_{j}^{n}=\Delta_{h} u_{j}^{n}, & 1 \leq j \leq J, \quad 0 \leq n \leq N \\ u_{0}^{n}=u_{J+1}^{n}=0, & 0 \leq n \leq N \\ \left(u_{j}^{0}+u_{j}^{1}\right) / 2=u_{0 j}, \quad\left(u_{j}^{1}-u_{j}^{0}\right) / \Delta t=u_{1 j}, & 0 \leq j \leq J+1 .\end{cases}
$$

which takes the following vectorial form

$$
\left\{\begin{array}{c}
M_{0}^{\theta_{\nu}}\left(\boldsymbol{U}_{\boldsymbol{h}}^{\boldsymbol{n}+\mathbf{1}}-2 \boldsymbol{U}_{\boldsymbol{h}}^{\boldsymbol{n}}+\boldsymbol{U}_{\boldsymbol{h}}^{\boldsymbol{n}-\mathbf{1}}\right)+\nu^{2} K \boldsymbol{U}_{\boldsymbol{h}}^{\boldsymbol{n}}=0 \quad n=0, \ldots, N, \\
\frac{\boldsymbol{U}_{\boldsymbol{h}}^{\mathbf{0}}+\boldsymbol{U}_{\boldsymbol{h}}^{\mathbf{1}}}{2}=\boldsymbol{u}_{\mathbf{0} \boldsymbol{h}}, \frac{\boldsymbol{U}_{\boldsymbol{h}}^{\mathbf{1}}-\boldsymbol{U}_{\boldsymbol{h}}^{\mathbf{0}}}{\Delta t}=\boldsymbol{u}_{\mathbf{1} \boldsymbol{h}}
\end{array}\right.
$$

where $\boldsymbol{U}_{\boldsymbol{h}}^{\boldsymbol{n}}=\left(u_{1}^{n}, \ldots, u_{J}^{n}\right)^{T}$.

\subsection{Study of the scheme $\left(\mathcal{A}_{h, \Delta t}^{\theta, \alpha}\right)$}

Definition 3.1. The discrete energy $E_{n}^{\theta, \alpha}, n=0, \ldots, N$, associated to the scheme (39) is

$$
E_{n}^{\theta, \alpha}=\frac{h}{2} \sum_{j=0}^{J}\left[\left(\frac{u_{j}^{n+1}-u_{j}^{n}}{\Delta t}\right)^{2}-\theta_{\nu}\left[\left(\frac{u_{j+1}^{n+1}-u_{j+1}^{n}}{\Delta t}\right)-\left(\frac{u_{j}^{n+1}-u_{j}^{n}}{\Delta t}\right)\right]^{2}+\left(\frac{u_{j+1}^{n+1}-u_{j}^{n+1}}{h}\right)\left(\frac{u_{j+1}^{n}-u_{j}^{n}}{h}\right)\right]
$$

Remark 3.2. We have the important equalities

$$
\begin{aligned}
E_{n}^{\theta, \alpha} & =\frac{1}{2}\left(K_{h} \boldsymbol{U}_{\boldsymbol{h}}^{n+1}, \boldsymbol{U}_{\boldsymbol{h}}^{\boldsymbol{n}}\right)+\frac{1}{2}\left(\left(I-\theta_{\nu} K\right) \frac{\boldsymbol{U}_{\boldsymbol{h}}^{\boldsymbol{n}+\mathbf{1}}-\boldsymbol{U}_{\boldsymbol{h}}^{\boldsymbol{n}}}{\Delta t}, \frac{\boldsymbol{U}_{\boldsymbol{h}}^{\boldsymbol{n}+\mathbf{1}}-\boldsymbol{U}_{\boldsymbol{h}}^{\boldsymbol{n}}}{\Delta t}\right) \\
& =\frac{1}{2}\left(\left(\boldsymbol{U}_{\boldsymbol{h}}^{\boldsymbol{n}+\mathbf{1}}, \frac{\boldsymbol{U}_{\boldsymbol{h}}^{\boldsymbol{n}+\mathbf{1}}-\boldsymbol{U}_{\boldsymbol{h}}^{\boldsymbol{n}}}{\Delta t}\right),\left(\boldsymbol{U}_{\boldsymbol{h}}^{\boldsymbol{n}}, \frac{\boldsymbol{U}_{\boldsymbol{h}}^{\boldsymbol{n}+\mathbf{1}}-\boldsymbol{U}_{\boldsymbol{h}}^{\boldsymbol{n}}}{\Delta t}\right)\right)_{0}=\frac{1}{2}\left\|\left(\frac{\boldsymbol{U}_{\boldsymbol{h}}^{\boldsymbol{n}+\mathbf{1}}+\boldsymbol{U}_{\boldsymbol{h}}^{\boldsymbol{n}}}{2}, \frac{\boldsymbol{U}_{\boldsymbol{h}}^{\boldsymbol{n}+\mathbf{1}}-\boldsymbol{U}_{\boldsymbol{h}}^{\boldsymbol{n}}}{\Delta t}\right)\right\|_{1}^{2}
\end{aligned}
$$

The following proposition indicates that the discrete system (39) is conservative:

Proposition 3.3. The energy $E_{n}^{\theta, \alpha}$ is constant in time:

$$
E_{n}^{\theta, \alpha}=E_{0}^{\theta, \alpha}, \quad \forall n=1, \ldots, N, \quad \forall \theta, \alpha \geq 0 .
$$


Proof. Multiplying the first equation of 40 by $\left(\boldsymbol{U}_{\boldsymbol{h}}^{\boldsymbol{n}+\boldsymbol{1}}-\boldsymbol{U}_{\boldsymbol{h}}^{\boldsymbol{n}-\mathbf{1}}\right)$ and using the symmetry of $K$ and $M_{0}^{\theta, \nu}$, we obtain the relation

$$
\begin{aligned}
\left(M_{0}^{\theta, \nu}\left(\boldsymbol{U}_{\boldsymbol{h}}^{n+1}-\boldsymbol{U}_{\boldsymbol{h}}^{\boldsymbol{n}}\right),\left(\boldsymbol{U}_{\boldsymbol{h}}^{\boldsymbol{n}+\mathbf{1}}-\boldsymbol{U}_{\boldsymbol{h}}^{\boldsymbol{n}}\right)\right)+\nu^{2}\left(K \boldsymbol{U}_{\boldsymbol{h}}^{\boldsymbol{n}+\boldsymbol{1}}, \boldsymbol{U}_{\boldsymbol{h}}^{\boldsymbol{n}}\right)= \\
\quad\left(M_{0}^{\theta, \nu}\left(\boldsymbol{U}_{\boldsymbol{h}}^{\boldsymbol{n}}-\boldsymbol{U}_{\boldsymbol{h}}^{\boldsymbol{n}-\mathbf{1}}\right),\left(\boldsymbol{U}_{\boldsymbol{h}}^{\boldsymbol{n}}-\boldsymbol{U}_{\boldsymbol{h}}^{n-1}\right)\right)+\nu^{2}\left(K \boldsymbol{U}_{\boldsymbol{h}}^{n}, \boldsymbol{U}_{\boldsymbol{h}}^{\boldsymbol{n}-\mathbf{1}}\right) .
\end{aligned}
$$

Then, multiplying by $1 /\left(2 \Delta t^{2}\right)$ and using the equality of (42), we obtain

$$
E_{n}^{\theta, \alpha}=E_{n-1}^{\theta, \alpha}, \quad \forall n=1, \ldots, N,
$$

leading to $E_{n}^{\theta, \alpha}=E_{0}^{\theta, \alpha}, \forall n=1 \ldots N$. This completes the proof of Proposition 3.3.

Lemma 3.4. For all $\alpha, \theta>0$, we have

$$
E_{n}^{\theta, \alpha} \geq \frac{1}{4 h} \min \left(1, \frac{1-4 \theta_{\nu}}{\nu^{2}}\right) \sum_{j=0}^{J}\left[\left(u_{j}^{n+1}-u_{j+1}^{n}\right)^{2}+\left(u_{j+1}^{n+1}-u_{j}^{n}\right)^{2}\right] .
$$

Proof. Let us first consider the case $\theta_{\nu} \geq 0$. We first write that

$$
E_{n}^{\theta, \alpha} \geq \frac{h}{2} \sum_{j=0}^{J}\left[\left(\frac{u_{j}^{n+1}-u_{j}^{n}}{\Delta t}\right)^{2}-2 \theta_{\nu}\left[\left(\frac{u_{j+1}^{n+1}-u_{j+1}^{n}}{\Delta t}\right)^{2}+\left(\frac{u_{j}^{n+1}-u_{j}^{n}}{\Delta t}\right)^{2}\right]+\left(\frac{u_{j+1}^{n+1}-u_{j}^{n+1}}{h}\right)\left(\frac{u_{j+1}^{n}-u_{j}^{n}}{h}\right)\right] .
$$

Then, using the Dirichlet boundary condition, it follows that

$$
E_{n}^{\theta, \alpha} \geq \frac{h}{2} \sum_{j=0}^{J}\left[\left(1-4 \theta_{\nu}\right)\left(\frac{u_{j}^{n+1}-u_{j}^{n}}{\Delta t}\right)^{2}+\left(\frac{u_{j+1}^{n+1}-u_{j}^{n+1}}{h}\right)\left(\frac{u_{j+1}^{n}-u_{j}^{n}}{h}\right)\right],
$$

and then,

$$
\begin{aligned}
E_{n}^{\theta, \alpha} & \geq \frac{1}{2 h} \sum_{j=0}^{J}\left[\frac{1-4 \theta_{\nu}}{\nu^{2}}\left(u_{j}^{n+1}-u_{j}^{n}\right)^{2}+\left(u_{j+1}^{n+1}-u_{j}^{n+1}\right)\left(u_{j+1}^{n}-u_{j}^{n}\right)\right] \\
& \geq \frac{1}{2 h} \min \left(1, \frac{1-4 \theta_{\nu}}{\nu^{2}}\right) \sum_{j=0}^{J}\left[\left(u_{j}^{n+1}-u_{j}^{n}\right)^{2}+\left(u_{j+1}^{n+1}-u_{j}^{n+1}\right)\left(u_{j+1}^{n}-u_{j}^{n}\right)\right] .
\end{aligned}
$$

Finally, simple computations (see for instance [20]) lead to

$$
\sum_{j=0}^{J}\left[\left(u_{j}^{n+1}-u_{j}^{n}\right)^{2}+\left(u_{j+1}^{n+1}-u_{j}^{n+1}\right)\left(u_{j+1}^{n}-u_{j}^{n}\right)\right]=\frac{1}{2} \sum_{j=0}^{J}\left[\left(u_{j}^{n+1}-u_{j+1}^{n}\right)^{2}+\left(u_{j+1}^{n+1}-u_{j}^{n}\right)^{2}\right],
$$

leading to the result. Finally, in the case $\theta_{\nu}<0$, we have $\min \left(1,\left(1-4 \theta_{\nu}\right) / \nu^{2}\right)=1$ and the result is obvious. This lemma implies the following proposition.

Proposition 3.5. For all $(\theta, \alpha, \nu) \in \mathcal{S}$, the energy satisfies:

$$
E_{n}^{\theta, \alpha}=0 \quad \Longleftrightarrow \quad\left(u_{j}^{n}\right)_{j}=0
$$

Proof. $(\theta, \alpha, \nu) \in \mathcal{S}$ implies that $\left(1-4 \theta_{\nu}\right) / \nu^{2}>1-\nu^{-2} \tan ^{2}(\pi h / 2)>0$ for $h>0$ small enough. Then, according to Lemma 3.4 , if the energy vanishes, we have that $u_{j}^{n+1}=u_{j+1}^{n}$ and $u_{j+1}^{n+1}=u_{j}^{n}$ for all $j=0, \ldots, J$. Then, 
taking into account that $u_{0}^{n}=u_{J+1}^{n}=0$, we get the result. According to Remark 3.2, $\|.\|_{1}$ is then a natural norm associated to the energy $E_{n}^{\theta, \alpha}$.

Proposition 3.6 (stability). The scheme $\left(\mathcal{A}_{h, \Delta t}^{\theta, \alpha}\right)$ is stable if and only if $(\theta, \alpha, \nu) \in \mathcal{S}$.

Proof. The scheme $\left(\mathcal{A}_{h, \Delta t}^{\theta, \alpha}\right)$ is stable if $E_{0}^{\theta, \alpha}$ is a positive quadratic form, i.e., if the matrix $M_{1}^{\theta_{\nu}}$ is positive definite (we recall that the matrix $K_{h}$ is positive definite). The eigenvalues $0<\lambda_{1}^{K}<\lambda_{2}^{K}<\ldots<\lambda_{J}^{K}$ of the matrix $K$ are given by $\lambda_{j}^{K}=4 \sin ^{2}(j \pi h / 2)$. This implies that the eigenvalues of $M_{1}^{\theta_{\nu}}=I-\left(\theta_{\nu}+\nu^{2} / 4\right) K$ are given by

$$
\lambda_{j}^{M_{1}^{\theta_{\nu}}}=1-4\left(\theta_{\nu}+\frac{\nu^{2}}{4}\right) \sin ^{2}\left(\frac{j \pi h}{2}\right), \quad 1 \leq j \leq J .
$$

Then, the matrix is positive definite if $\max _{1 \leq j \leq J} \lambda_{j}^{M_{1}^{\theta_{\nu}}}>0$, implying $\max _{1 \leq j \leq J} \sin ^{2}(k \pi h / 2)\left(\nu^{2}(1-4 \alpha)+4 \theta\right)<1$ and finally the condition $(\theta, \alpha, \nu) \in \mathcal{S}$ using $J=1 / h-1$.

Proposition 3.7 (consistency). For all $\theta, \alpha \geq 0$, the error of consistency produced by the scheme $\left(\mathcal{A}_{h, \Delta t}^{\theta, \alpha}\right)$ is of order

$$
(\theta-1 / 12) O\left(h^{2}\right)+(\alpha-1 / 12) O\left(\Delta t^{2}\right)+O\left(h^{4}\right)+O\left(\Delta t^{4}\right)+O\left(h^{2} \Delta t^{2}\right) .
$$

Proof. Formally, using Taylor developments, we obtain

$$
\begin{aligned}
(1 & \left.+\theta h^{2} \Delta_{h}\right) \Delta_{\Delta t} u\left(x_{j}, t_{n}\right)=u_{t t}\left(x_{j}, t_{n}\right)+\frac{1}{12} \Delta t^{2} u_{t t t t}\left(x_{j}, t_{n}\right) \\
& +\theta h^{2} u_{t t x x}\left(x_{j}, t_{n}\right)+O\left(h^{2} \Delta t^{2}\right)+O\left(h^{4}\right)+O\left(\Delta t^{4}\right), \\
\left(1+\alpha \Delta t^{2} \Delta_{\Delta t}\right) \Delta_{h} u\left(x_{j}, t_{n}\right)=u_{x x}\left(x_{j}, t_{n}\right)+\frac{1}{12} h^{2} u_{x x x x}\left(x_{j}, t_{n}\right) & \\
& +\alpha \Delta t^{2} u_{x x t t}\left(x_{j}, t_{n}\right)+O\left(h^{2} \Delta t^{2}\right)+O\left(h^{4}\right)+O\left(\Delta t^{4}\right),
\end{aligned}
$$

leading to

$$
\begin{aligned}
& \left.\left[\left(1+\theta h^{2} \Delta_{h}\right) \Delta_{\Delta t}-\left(1+\alpha \Delta t^{2} \Delta_{\Delta t}\right) \Delta_{h}\right)\right] u\left(x_{j}, t_{n}\right)-\left[u_{t t}-u_{x x}\right]\left(x_{j}, t_{n}\right)= \\
& \quad h^{2}\left[\theta u_{t t x x}\left(x_{j}, t_{n}\right)-\frac{1}{12} u_{x x x x}\left(x_{j}, t_{n}\right)\right]+\Delta t^{2}\left[\frac{1}{12} u_{t t t t}\left(x_{j}, t_{n}\right)-\alpha u_{x x t t}\left(x_{j}, t_{n}\right)\right]+O\left(h^{2} \Delta t^{2}\right)+O\left(h^{4}\right)+O\left(\Delta t^{4}\right) .
\end{aligned}
$$

Then, using $u_{t t}\left(x_{j}, t_{n}\right)-u_{x x}\left(x_{j}, t_{n}\right)=0$, we formally obtain that

$$
\begin{aligned}
& \left.\left[\left(1+\theta h^{2} \Delta_{h}\right) \Delta_{\Delta t}-\left(1+\alpha \Delta t^{2} \Delta_{\Delta t}\right) \Delta_{h}\right)\right] u\left(x_{j}, t_{n}\right)-\left[u_{t t}-u_{x x}\right]\left(x_{j}, t_{n}\right)= \\
& \quad\left[h^{2}\left(\theta-\frac{1}{12}\right)+\Delta t^{2}\left(\frac{1}{12}-\alpha\right)\right] u_{t t t t}\left(x_{j}, t_{n}\right)+O\left(h^{2} \Delta t^{2}\right)+O\left(h^{4}\right)+O\left(\Delta t^{4}\right)
\end{aligned}
$$

Equation (56) then gives the result. Finally, it appears also that for $\theta=\alpha$ and $\Delta t=h$ leading to $\theta_{\nu}=0$ and to the scheme $\left(\mathcal{A}_{h, h}^{\theta, \theta}\right)$, the consistency is arbitrarily large.

Remark 3.8. From (56), we remark that for $\nu=\sqrt{(1-12 \theta) /(1-12 \alpha)}$, the consistency is fourth order in time and space. However, in this case $(\theta, \alpha, \nu) \notin \mathcal{C}$.

The consistency and the stability of the scheme $\left(\mathcal{A}_{h, \Delta t}^{\theta, \alpha}\right)$ imply, using the Lax theory, the convergence in a suitable norm of the sequence $\left(\boldsymbol{U}_{\boldsymbol{h}}\right)_{h, \Delta t}$ towards $u$ solution of the system $(\mathcal{A})$ when $h, \Delta t$ go to zero. 


\subsection{Uniform discrete observability inequality}

The aim of this section is to prove the following fundamental theorem.

Theorem 3.9. Let $(\theta, \alpha, \nu) \in \mathcal{C}$ and $T>2 \max \left(1, \nu^{2}\right)$. There exist two constants $C_{1}, C_{2}>0$ independent of $h$ and $\Delta t$ such that

$$
C_{1} E_{0}^{\theta, \alpha} \leq \Delta t \sum_{n=0}^{N-1}\left(\left|\frac{u_{J}^{n}}{h}\right|^{2}+\theta_{\nu}\left|\frac{u_{J}^{n+1}-u_{J}^{n}}{\Delta t}\right|^{2}\right) \leq C_{2} E_{0}^{\theta, \alpha}
$$

The left part of (57) is a uniform discrete observability inequality, discrete version of the inequality

$$
E(0) \leq C \int_{0}^{T}\left[\left(u_{x}(1, t)\right)^{2}+p \theta_{\nu}\left(u_{x}^{\prime}(1, t)\right)^{2}\right] \mathrm{d} t
$$

with $p=h^{2}$, similar to (8). It is possible to prove these inequalities using a discrete multiplier technique (see for instance [14] in the continuous case and [19] in the discrete case). However, for simplicity, we present a proof based on Fourier analysis. In this respect, we assume that the solution $\boldsymbol{U}_{\boldsymbol{h}}^{\boldsymbol{n}}$ takes the following form

$$
\boldsymbol{U}_{\boldsymbol{h}}^{\boldsymbol{n}}=\sum_{k=1}^{J}\left[a_{k, h} \cos \left(\sqrt{\lambda_{k, h}^{\theta, \alpha}} n \Delta t\right)+\frac{b_{k, h}}{\sqrt{\lambda_{k, h}^{\theta, \alpha}}} \sin \left(\sqrt{\lambda_{k, h}^{\theta, \alpha}} n \Delta t\right)\right] \boldsymbol{\phi}_{\boldsymbol{k}, \boldsymbol{h}}
$$

or equivalently

$$
\boldsymbol{U}_{\boldsymbol{h}}^{\boldsymbol{n}}=\sum_{|k| \leq J, k \neq 0} c_{k, h} \mathrm{e}^{i \mu_{k, h} n \Delta t} \boldsymbol{\phi}_{\boldsymbol{k}, \boldsymbol{h}}
$$

where

$$
\mu_{-k, h}=-\mu_{k, h} ; \mu_{k, h}=\sqrt{\lambda_{k, h}^{\theta, \alpha}} ; c_{k, h}=\frac{a_{k, h}-i b_{k, h} / \mu_{k, h}}{2} ; c_{-k, h}=\overline{c_{k, h}} .
$$

Let us first show the

Proposition 3.10. Let $(\theta, \alpha, \nu) \in \mathcal{C}$. For every $T>2 \max \left(1, \nu^{2}\right)$, there exist two constants $c, C>0$ independent of $\Delta t$ and $h$ such that

$$
\begin{aligned}
c(T) \min \left(1, \nu^{-2}\right) \sum_{|k| \leq J, k \neq 0}\left|c_{k, h}\right|^{2}\left|\frac{\sin (k \pi h)}{h}\right|^{2} & \leq \Delta t \sum_{n=0}^{N-1}\left(\left|\frac{u_{J}^{n}}{h}\right|^{2}+\theta_{\nu}\left|\frac{u_{J}^{n+1}-u_{J}^{n}}{\Delta t}\right|^{2}\right) \\
& \leq C(T) \max \left(1, \nu^{-2}\right) \sum_{|k| \leq J, k \neq 0}\left|c_{k, h}\right|^{2}\left|\frac{\sin (k \pi h)}{h}\right|^{2}
\end{aligned}
$$

In order to show these inequalities, we use the following discrete version of Ingham's inequality for nonharmonic Fourier series [13] proved in [21].

Theorem 3.11 (discrete Ingham inequality). Let $\Delta t>0$ and $\left\{\mu_{k}\right\}$ be a sequence of reel numbers satisfying for some $\gamma$ and $0 \leq p<1 / 2$ the conditions:

$$
\begin{aligned}
& \mu_{k+1}-\mu_{k} \geq \gamma>0, \quad \forall k \in \mathbb{Z}, \\
& \left|\mu_{k}-\mu_{l}\right| \leq \frac{2 \pi-(\Delta t)^{p}}{\Delta t}, \quad \forall k, l \in \mathbb{Z} .
\end{aligned}
$$


Then, for every $T>2 \pi / \gamma$, there exist two positives constants $C_{1}(T, \gamma)$ and $C_{2}(T, \gamma)$ such that

$$
C_{1}(T, \gamma) \sum_{k=-J}^{J}\left|c_{k}\right|^{2} \leq \Delta t \sum_{n=0}^{N-1} \sum_{k=-J}^{J}\left|c_{k} \mathrm{e}^{i n \Delta t \mu_{k}}\right|^{2} \leq C_{2}(T, \gamma) \sum_{k=-J}^{J}\left|c_{k}\right|^{2}
$$

for every complex sequence $\left(c_{k}\right)_{k \in Z} \in l^{2}$.

If $(\theta, \alpha, \nu) \in \mathcal{C}$, the conditions $(63)$ are fullfiled:

Lemma 3.12. Let $(\theta, \alpha, \nu) \in \mathcal{C}$. Then,

$$
\begin{aligned}
& \sqrt{\lambda_{j, h}^{\theta, \alpha}}-\sqrt{\lambda_{j-1, h}^{\theta, \alpha}} \geq \pi \min \left(1, \nu^{-2}\right), \quad \forall j=2, \ldots, J \\
& \left|\sqrt{\lambda_{j, h}^{\theta, \alpha}}-\sqrt{\lambda_{k, h}^{\theta, \alpha}}\right| \leq 2 \pi \Delta t^{-1}+O(1), \quad \forall j, k=1, \ldots, J .
\end{aligned}
$$

Proof. The first inequality is a consequence of the following relation obtained using a Taylor expansion. For instance, for $(\theta, \alpha, \nu) \in \mathcal{C}_{2}$, we obtain:

- $\quad \sqrt{\lambda_{k, h}^{\theta, \alpha}}-\sqrt{\lambda_{k-1, h}^{\theta, \alpha}}=\pi+O\left(h^{2}\right), \quad \forall k \ll J ;$

- $\sqrt{\lambda_{\beta J, h}^{\theta, \alpha}}-\sqrt{\lambda_{\beta J-1, h}^{\theta, \alpha}}=\pi \frac{1-4 \alpha}{1-4 \theta+4(\theta-\alpha) \cos ^{2}\left(\frac{\beta \pi}{2}\right)}+O\left(h^{2}\right), \quad \forall 0 \leq \beta \leq 1$.

The second inequality, less restrictive, is a consequence of the following relations

- $\sqrt{\lambda_{k, h}^{\theta, \alpha}}-\sqrt{\lambda_{l, h}^{\theta, \alpha}}=(k-l) \pi+O\left(h^{2}\right), \quad \forall k, l \ll J ;$

- $\sqrt{\lambda_{\beta_{1} J, h}^{\theta, \alpha}}-\sqrt{\lambda_{\beta_{2} J, h}^{\theta, \alpha}}=2 \frac{\sqrt{1-4 \alpha}}{\sqrt{1-4 \theta}}\left(f\left(\beta_{1}\right)-f\left(\beta_{2}\right)\right) h^{-1}+O(1), \quad \forall 0 \leq \beta_{1}, \beta_{2} \leq 1$

$$
=2\left(f\left(\beta_{1}\right)-f\left(\beta_{2}\right)\right) \Delta t^{-1}+O(1),
$$

where

$$
f(\beta)=\arcsin \left(\frac{\sqrt{1-4 \theta} \sin \left(\frac{\beta \pi}{2}\right)}{\sqrt{1-4 \theta+4(\theta-\alpha) \cos ^{2}\left(\frac{\pi \beta}{2}\right)}}\right)
$$

using $|\arcsin (x)| \leq \pi / 2, \forall x \in[0,1]$.

Thanks to this lemma, we are now in position to prove the Proposition 3.10.

Proof of Proposition 3.10. It results from (60) that

$$
\Delta t \sum_{n=0}^{N-1}\left|\frac{u_{J}^{n}}{h}\right|^{2}=\Delta t \sum_{n=0}^{N-1}\left|\sum_{|k| \leq J, k \neq 0} c_{k, h} \mathrm{e}^{i \mu_{k, h} n \Delta t} \frac{\sin (k \pi J h)}{h}\right|^{2} .
$$

Then, according to Lemma 3.12, we can apply Theorem 3.11 with $p=0$ and $T>2 \max \left(1, \nu^{2}\right)$ to obtain that there exist two constants $C_{1}$ and $C_{2}$ independent of $\Delta t$ and therefore $h$ such that

$$
C_{1}(T) \sum_{|k| \leq J, k \neq 0}\left|c_{k, h}\right|^{2}\left|\frac{\sin (k \pi J h)}{h}\right|^{2} \leq \Delta t \sum_{n=0}^{N-1}\left|\frac{u_{J}^{n}}{h}\right|^{2} \leq C_{2}(T) \sum_{|k| \leq J, k \neq 0}\left|c_{k, h}\right|^{2}\left|\frac{\sin (k \pi J h)}{h}\right|^{2}
$$


Then, we compute

$$
\frac{u_{J}^{n+1}-u_{J}^{n}}{\Delta t}=\sum_{|k| \leq J, k \neq 0} \tilde{c}_{k, h} \mathrm{e}^{i \mu_{k, h} n \Delta t} \sin (k \pi J h)
$$

with

$$
\tilde{c}_{k, h}=c_{k, h} \frac{\mathrm{e}^{i \mu_{k, h} \Delta t}-1}{\Delta t}
$$

Therefore, applying again the Theorem 3.11, there exist two constants $C_{3}$ and $C_{4}$ such that the following inequalities hold:

$$
C_{3}(T) \sum_{|k| \leq J, k \neq 0}\left|\tilde{c}_{k, h}\right|^{2}|\sin (k \pi J h)|^{2} \leq \Delta t \sum_{n=0}^{N-1}\left|\frac{u_{J}^{n+1}-u_{J}^{n}}{\Delta t}\right|^{2} \leq C_{4}(T) \sum_{|k| \leq J, k \neq 0}\left|\tilde{c}_{k, h}\right|^{2}|\sin (k \pi J h)|^{2} .
$$

Finally, from (70) and (73), there exist two constants such that

$$
\begin{array}{r}
c(T) \sum_{|k| \leq J, k \neq 0}\left(\left|c_{k, h}\right|^{2}+\theta_{\nu} h^{2}\left|\tilde{c}_{k, h}\right|^{2}\right)\left|\frac{\sin (k \pi J h)}{h}\right|^{2} \leq \Delta t \sum_{n=0}^{N-1}\left(\left|\frac{u_{J}^{n}}{h}\right|^{2}+\theta_{\nu}\left|\frac{u_{J}^{n+1}-u_{J}^{n}}{\Delta t}\right|^{2}\right) \\
\leq C(T) \sum_{|k| \leq J, k \neq 0}\left(\left|c_{k, h}\right|^{2}+\theta_{\nu} h^{2}\left|\tilde{c}_{k, h}\right|^{2}\right)\left|\frac{\sin (k \pi J h)}{h}\right|^{2} .
\end{array}
$$

Let us then show that

$$
\min \left(1, \nu^{-2}\right)\left|c_{k, h}\right|^{2} \leq\left(\left|c_{k, h}\right|^{2}+\theta_{\nu} h^{2}\left|\tilde{c}_{k, h}\right|^{2}\right) \leq \max \left(1, \nu^{-2}\right)\left|c_{k, h}\right|^{2} .
$$

We write $\left(\left|c_{k, h}\right|^{2}+\theta_{\nu} h^{2}\left|\tilde{c}_{k, h}\right|^{2}\right)=\left|c_{k, h}\right|^{2}\left(1+\theta_{\nu} \nu^{-2}\left|\mathrm{e}^{i \mu_{k, h} \Delta t}-1\right|^{2}\right)$. In the case $\theta_{\nu}>0$, using $\left|\mathrm{e}^{i \mu_{k, h} \Delta t}-1\right|^{2} \leq 4$, we obtain that

$$
\left|c_{k, h}\right|^{2} \leq\left(\left|c_{k, h}\right|^{2}+\theta_{\nu} h^{2}\left|\tilde{c}_{k, h}\right|^{2}\right) \leq\left|c_{k, h}\right|^{2}\left(1+4 \theta_{\nu} \nu^{-2}\right)
$$

whereas in the case $\theta_{\nu}<0$, we obtain

$$
\left|c_{k, h}\right|^{2}\left(1+4 \theta_{\nu} \nu^{-2}\right) \leq\left(\left|c_{k, h}\right|^{2}+\theta_{\nu} h^{2}\left|\tilde{c}_{k, h}\right|^{2}\right) \leq\left|c_{k, h}\right|^{2} .
$$

Then, for $(\theta, \alpha, \nu) \in \mathcal{C}_{1}$, we have $\left(1+4 \theta_{\nu} \nu^{-2}\right)=\left(1+\left(1-\nu^{2}\right) \nu^{-2}\right)=\nu^{-2}>0$, whereas for $(\theta, \alpha, \nu) \in \mathcal{C}_{\mathbf{2}}$, we have $\left(1+4 \theta_{\nu} \nu^{-2}\right)=1+4 \frac{\theta-\alpha}{1-4 \alpha} \frac{1-4 \alpha}{1-4 \theta}=\frac{1-4 \alpha}{1-4 \theta}=\nu^{-2}$, leading to (75). Finally, from (74) and (75) we obtain the result by noting that $\sin (k \pi J h)=-\sin (k \pi h)$ by definition of $h=1 /(J+1)$, leading to $J=1 / h-1$.

We now evaluate the energy $E_{0}^{\theta, \alpha}$. We have:

Theorem 3.13. Let $(\theta, \alpha, \nu) \in \mathcal{C}$. Then, we have the following inequalities:

$$
\min \left(1, \nu^{-2}\right) \sum_{|k| \leq J, k \neq 0}\left|c_{k, h}\right|^{2}\left|\frac{\sin (k \pi h)}{h}\right|^{2} \leq 2 E_{0}^{\theta, \alpha} \leq \max \left(1, \nu^{-2}\right) \sum_{|k| \leq J, k \neq 0}\left|c_{k, h}\right|^{2}\left|\frac{\sin (k \pi h)}{h}\right|^{2} .
$$

Proof. According to (42), we have

$$
E_{0}^{\theta, \alpha}=\frac{1}{8 h^{2}}\left(K\left(\boldsymbol{U}_{\boldsymbol{h}}^{1}+\boldsymbol{U}_{\boldsymbol{h}}^{\mathbf{0}}\right), \boldsymbol{U}_{\boldsymbol{h}}^{\mathbf{1}}+\boldsymbol{U}_{\boldsymbol{h}}^{\mathbf{0}}\right)+\frac{1}{2 \Delta t^{2}}\left(M_{1}^{\theta_{\nu}}\left(\boldsymbol{U}_{\boldsymbol{h}}^{\mathbf{1}}-\boldsymbol{U}_{\boldsymbol{h}}^{\mathbf{0}}\right), \boldsymbol{U}_{\boldsymbol{h}}^{\mathbf{1}}-\boldsymbol{U}_{\boldsymbol{h}}^{\mathbf{0}}\right)
$$


Then using that

$$
\begin{aligned}
& \boldsymbol{U}_{\boldsymbol{h}}^{\mathbf{1}}+\boldsymbol{U}_{\boldsymbol{h}}^{\mathbf{0}}=\sum_{|k| \leq J, k \neq 0} c_{k, h}\left(\mathrm{e}^{i \mu_{k} \Delta t}+1\right) \boldsymbol{\phi}_{\boldsymbol{k}, \boldsymbol{h}} \\
& \boldsymbol{U}_{\boldsymbol{h}}^{\mathbf{1}}-\boldsymbol{U}_{\boldsymbol{h}}^{\mathbf{0}}=\sum_{|k| \leq J, k \neq 0} c_{k, h}\left(\mathrm{e}^{i \mu_{k} \Delta t}-1\right) \boldsymbol{\phi}_{\boldsymbol{k}, \boldsymbol{h}} \equiv \sum_{|k| \leq J, k \neq 0} c_{1 k} \boldsymbol{\phi}_{\boldsymbol{k}, \boldsymbol{h}}, \\
& c_{2 k} \boldsymbol{\phi}_{\boldsymbol{k}, \boldsymbol{h}},
\end{aligned}
$$

we obtain

$$
E_{0}^{\theta, \alpha}=\frac{1}{8 h^{2}} \sum_{|k|,|q| \leq J, k \neq 0, q \neq 0} c_{1 k} c_{1 q}\left(K \boldsymbol{\phi}_{\boldsymbol{k}, \boldsymbol{h}}, \boldsymbol{\phi}_{\boldsymbol{q}, \boldsymbol{h}}\right)+\frac{1}{2 \Delta t^{2}} \sum_{|k|,|q| \leq J, k \neq 0, q \neq 0} c_{2 k} c_{2 q}\left(M_{1}^{\theta_{\nu}} \boldsymbol{\phi}_{\boldsymbol{k}, \boldsymbol{h}}, \boldsymbol{\phi}_{\boldsymbol{q}, \boldsymbol{h}}\right) .
$$

Furthermore, the eigenvalues of the matrix $K$ are $\lambda_{k}^{K}=4 \sin ^{2}(k \pi h / 2), k=1, \ldots, J$, associated to the eigenvectors $\phi_{h}^{k}$. Besides, $M_{1}^{\theta_{\nu}}=M_{0}^{\theta_{\nu}}-\nu^{2} / 4 K=I-\left(\theta_{\nu}+\nu^{2} / 4\right) K=I-K / 4$. This implies that

$$
E_{0}^{\theta, \alpha}=\frac{1}{8 h^{2}} \sum_{|k|,|q| \leq J, k \neq 0, q \neq 0} c_{1 k} c_{1 q} \lambda_{k}^{K}\left(\phi_{\boldsymbol{k}, \boldsymbol{h}}, \boldsymbol{\phi}_{\boldsymbol{q}, \boldsymbol{h}}\right)+\frac{1}{2 \Delta t^{2}} \sum_{|k|,|q| \leq J, k \neq 0, q \neq 0} c_{2 k} c_{2 q}\left(1-\frac{\lambda_{k}^{K}}{4}\right)\left(\boldsymbol{\phi}_{\boldsymbol{k}, \boldsymbol{h}}, \boldsymbol{\phi}_{\boldsymbol{q}, \boldsymbol{h}}\right) .
$$

Then, using that

we obtain

$$
\left(\phi_{\boldsymbol{k}, \boldsymbol{h}}, \boldsymbol{\phi}_{\boldsymbol{q}, \boldsymbol{h}}\right)=\frac{J+1}{2} \delta_{k q}=\frac{1}{2 h} \delta_{k q}
$$

$$
E_{0}^{\theta, \alpha}=\frac{1}{4 h^{2}} \sum_{|k| \leq J, k \neq 0}\left(\left|c_{1 k}\right|^{2} \sin ^{2}(k \pi h / 2)+\frac{1}{\nu^{2}}\left|c_{2 k}\right|^{2} \cos ^{2}(k \pi h / 2)\right) .
$$

Then, we write $\left|c_{2 k}\right|^{2}=2\left|c_{k, h}\right|^{2}\left(1-\cos \left(\mu_{k, h} \Delta t\right)\right)$ and $\left|c_{1 k}\right|^{2}=2\left|c_{k, h}\right|^{2}\left(1+\cos \left(\mu_{k, h} \Delta t\right)\right)$. We write that $\cos \left(\mu_{k, h} \Delta t\right)=1-2 \sin ^{2}\left(\mu_{k, h} \frac{\Delta t}{2}\right)$ to obtain

$$
\cos \left(\mu_{k, h} \Delta t\right)=1-2 \frac{\nu^{2} \sin ^{2}(k \pi h / 2)}{1-4 \theta_{\nu} \sin ^{2}(k \pi h / 2)}
$$

This leads to

$$
1+\cos \left(\mu_{k, h} \Delta t\right)=\frac{2 \cos ^{2}(k \pi h / 2)}{1-4 \theta_{\nu} \sin ^{2}(k \pi h / 2)}, \quad 1-\cos \left(\mu_{k, h} \Delta t\right)=\frac{2 \nu^{2} \sin ^{2}(k \pi h / 2)}{1-4 \theta_{\nu} \sin ^{2}(k \pi h / 2)},
$$

and finally to

$$
E_{0}^{\theta, \alpha}=2 \sum_{|k| \leq J, k \neq 0} \frac{\left|c_{k, h}\right|^{2}}{h^{2}} \frac{\sin ^{2}(k \pi h / 2) \cos ^{2}(k \pi h / 2)}{1-4 \theta_{\nu} \sin ^{2}(k \pi h / 2)}=\frac{1}{2} \sum_{|k| \leq J, k \neq 0} \frac{\left|c_{k, h}\right|^{2}}{h^{2}} \frac{\sin ^{2}(k \pi h)}{1-4 \theta_{\nu} \sin ^{2}(k \pi h / 2)} .
$$

Then using the fact that $1+4 \theta_{\nu} \nu^{-2}=\nu^{-2}$, we show that

$$
\min \left(1, \nu^{2}\right) \leq 1-4 \theta_{\nu} \sin ^{2}(k \pi h / 2) \leq \max \left(1, \nu^{2}\right)
$$

(we recall that $\nu=1$ implies $\theta_{\nu}=0$ when $(\theta, \alpha, \nu) \in \mathcal{C}$ ), leading to the result.

According to the Theorem 3.11, the uniform gap between two consecutive eigenvalues is a sufficient (and actually also necessary) property that leads to uniform observability inequalities with respect to $h$ and $\Delta t$. In the continuous case, this gap is constantly equal to $\pi$. Figures 3 and 4 depict the evolution of the square root of $\lambda_{k}^{\theta, \alpha}$ for different values of $\theta, \alpha$ and $\nu$. 


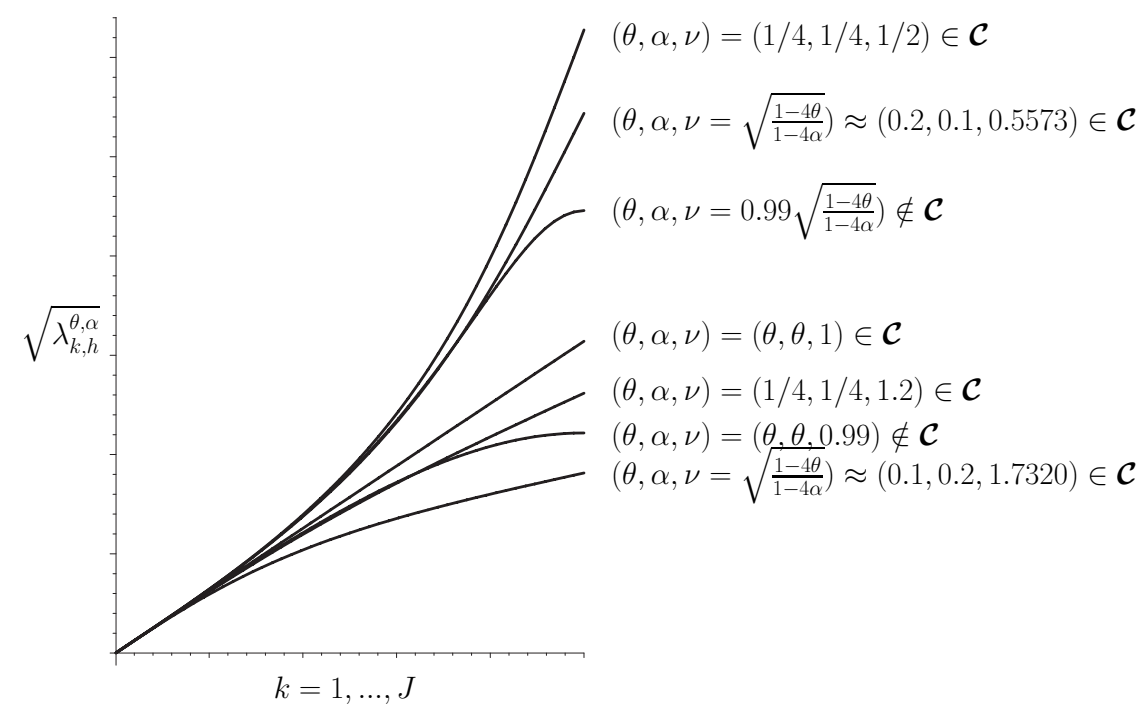

FiguRE 3. Evolution of $\sqrt{\lambda_{k, h}^{\theta, \alpha}}, k=1, \ldots, J$ for different values of $\theta, \alpha$ and $\nu$.

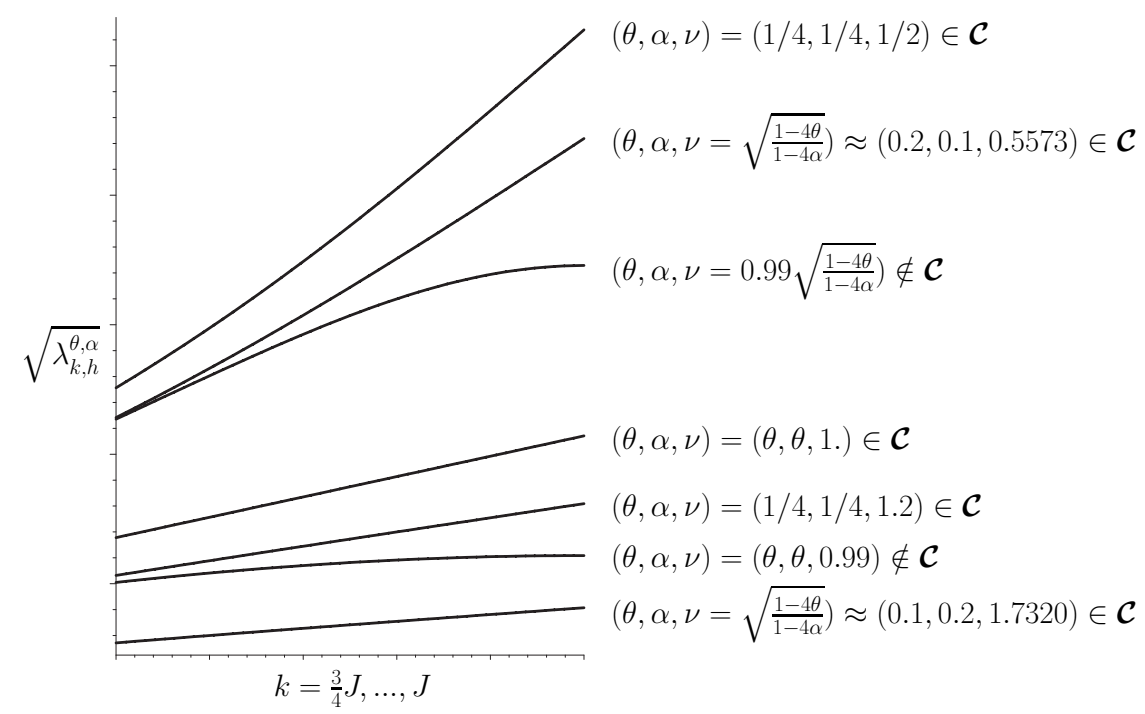

Figure 4. Evolution of $\sqrt{\lambda_{k, h}^{\theta, \alpha}}, k=\frac{3}{4} J, \ldots, J$ for different values of $\theta, \alpha$ and $\nu$ : zoom on the high frequencies.

Remark 3.14. According to the reversibility in time of the wave system, the previous inequalities obtained with $\boldsymbol{U}_{\boldsymbol{h}}$ solution of (40) are also true for $\boldsymbol{W}_{\boldsymbol{h}}$ solution of (21).

Remark 3.15. If we assume that $\nu$ may be not of order one, we may add to $\mathcal{C}$ the following subset of $\mathcal{S}$

$$
\mathcal{C}_{3}=\left\{\left(\theta, \alpha, \nu_{h}\right) \in \mathbb{R}^{+} \times \mathbb{R}^{+} \times \mathbb{R}_{\star}^{+}, \nu_{h}=\frac{\tan (\pi h / 2)}{\sqrt{1-4 \alpha}}, \alpha<1 / 4, \theta=1 / 4\right\} .
$$




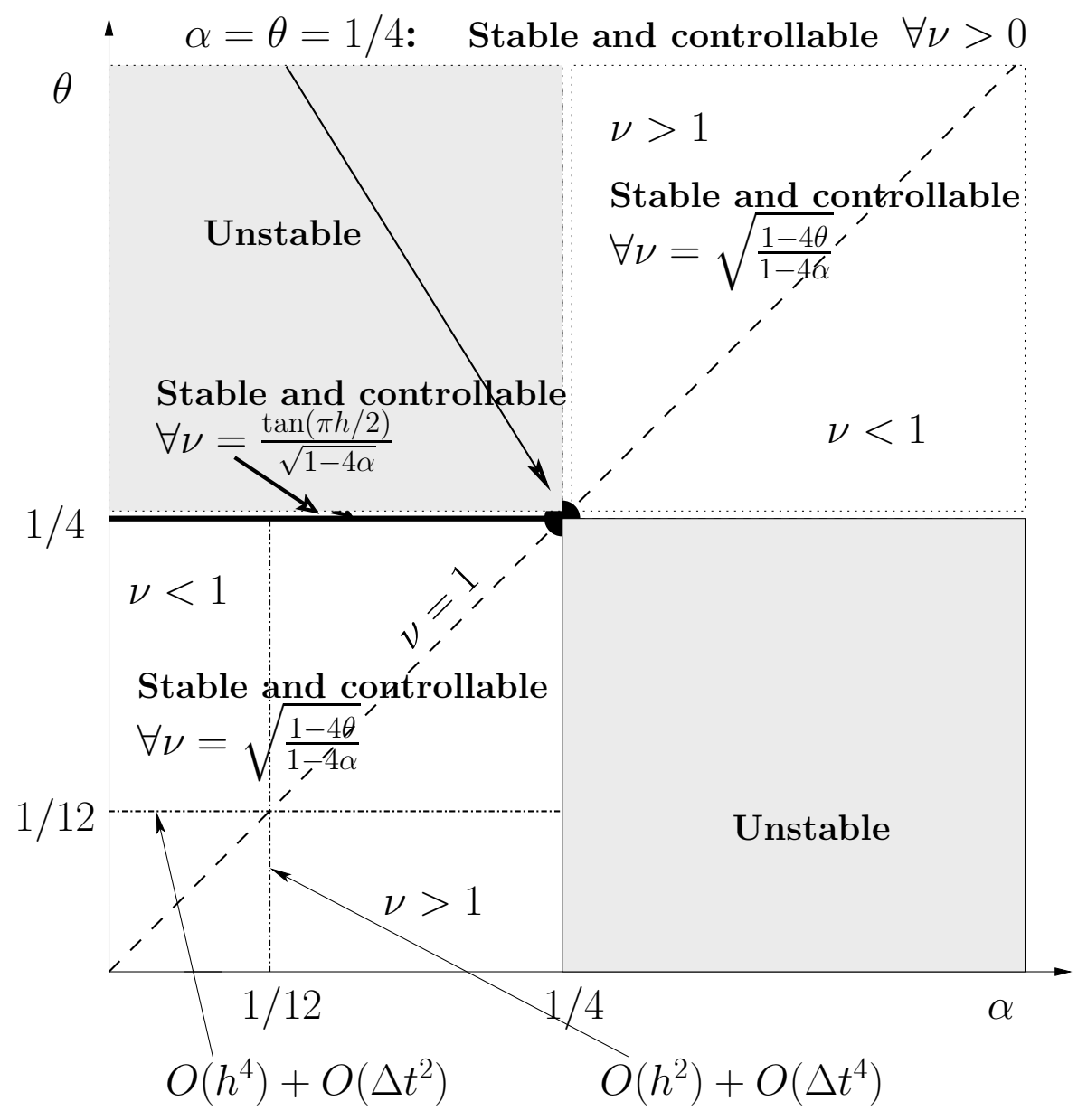

FiguRE 5. Zone of stability and uniform controllability for $\theta, \alpha \geq 0$ associated to the condition $T>2 \max \left(1, \nu^{2}\right)$.

Similar results of uniform controllability can be obtained with $\left(\theta, \alpha, \nu_{h}\right) \in \mathcal{C}_{\mathbf{3}}$ for $T>2 \max \left(1, \nu_{h}^{2}\right)=2$. In particular, the spectrum fulfills a uniform gap property. The major drawback is that $\Delta t$ is of order $h^{2}$, which produces a very time consuming scheme. Furthermore, if we assume that $\theta$ may depend on $h$, another subset that ensures a uniform gap property is the following one:

$$
\mathcal{C}_{\mathbf{4}}=\left\{\left(\theta_{h}, \alpha, \nu\right) \in \mathbb{R}^{+} \times \mathbb{R}^{+} \times \mathbb{R}_{\star}^{+}, \theta_{h}=(2 \cos (\pi h / 2))^{-2}, \alpha=1 / 4\right\}
$$

and also

$$
\mathcal{C}_{\mathbf{5}}=\left\{\left(\theta_{h}, \alpha, \nu_{h}\right) \in \mathbb{R}^{+} \times \mathbb{R}^{+} \times \mathbb{R}_{\star}^{+}, \nu_{h}=\sqrt{\frac{\cos (\pi h / 2)^{-2}-4 \theta_{h}}{1-4 \alpha}},\left(\cos (\pi h / 2)^{-2}-4 \theta_{h}\right)(1-4 \alpha)>0\right\}
$$

Numerical results obtained with $\mathcal{C}_{\mathbf{4}}\left(\right.$ resp. $\left.\mathcal{C}_{\mathbf{5}}\right)$ are very close to those obtained with $\mathcal{C}_{\mathbf{1}}\left(\right.$ resp. $\left.\mathcal{C}_{\mathbf{2}}\right)$. We summarize these different results in Figure 5. 


\subsection{A convergence result}

In this short paragraph, we prove a convergence result for the homogeneous adjoint system (32). We assume that the corresponding solution of (32) has the following Fourier decomposition

$$
\boldsymbol{W}_{\boldsymbol{h}}^{\boldsymbol{n}}=\sum_{k=1}^{J}\left[\underline{a}_{k, h} \cos \left(\mu_{k}(n-N) \Delta t\right)+\frac{\underline{b}_{k, h}}{\mu_{k, h}} \sin \left(\mu_{k}(n-N) \Delta t\right)\right] \boldsymbol{\phi}_{\boldsymbol{k}, \boldsymbol{h}}
$$

and that the solution of the continuous system (6) takes the form

$$
w(., t)=\sum_{k=1}^{J}\left[\underline{a}_{k} \cos (k \pi(t-T))+\frac{\underline{b}_{k}}{k \pi} \sin (k \pi(t-T))\right] \phi_{k} .
$$

Lemma 3.16. Let $\boldsymbol{W}_{\boldsymbol{h}}^{\boldsymbol{n}}$ and $w(., t)$ be given by (92) and (93) respectively. Assume that $\left(\underline{a}_{k, h}, \frac{\underline{b}_{k, h}}{\mu_{k, h}}\right)_{k}$ weakly converge toward $\left(\underline{a}_{k}, \frac{\underline{b}_{k}}{k \pi}\right)_{k}$ in $l^{2}$ when $h \rightarrow 0$. Then, the sequence $\left(Q\left(w_{N} / h\right)\right)_{h>0}$ converges weakly to $w_{x}(1,$.$) in$ $L^{2}(0, T)$ when $h$ tends to zero.

Proof. It suffices to see that for any test function $\psi \in \mathcal{D}(0, T)$,

$$
\int_{0}^{T} Q\left(\frac{w_{J}}{h}\right) \psi(t) \mathrm{d} t \rightarrow \int_{0}^{T} w_{x}(1, .) \psi(t) \mathrm{d} t
$$

But from $\left(\phi_{k}\right)^{\prime}(1)=(-1)^{k} k \pi=\lim _{h \rightarrow 0}\left(\phi_{J, k}\right) / h$ and the weak convergence of the Fourier coefficients, the result follows.

\section{The CONTROLlability PROBlem}

\subsection{Existence of the discrete controls. Proofs of Theorem 2.7}

Firstly, we deduce a variational characterization of the controllability property for the system (21). Let $\boldsymbol{W}_{\boldsymbol{h}}^{\boldsymbol{n}}$ be the solution of the adjoint backward homogeneous system (32). Multiplying the system (32) by the solution $\boldsymbol{Y}_{\boldsymbol{h}}^{\boldsymbol{n}}$ of system (21), summing for $n=1, \ldots, N-1$, we obtain the following relation:

$$
\sum_{n=1}^{N-1}\left\langle\boldsymbol{F}_{\boldsymbol{h}}^{\boldsymbol{n}}, \boldsymbol{W}_{\boldsymbol{h}}^{\boldsymbol{n}}\right\rangle+\left\langle\boldsymbol{W}_{\boldsymbol{h}}^{\boldsymbol{N}}, M_{0}^{\theta_{\nu}} \boldsymbol{Y}_{\boldsymbol{h}}^{\boldsymbol{N}-\mathbf{1}}\right\rangle-\left\langle\boldsymbol{W}_{\boldsymbol{h}}^{\mathbf{1}}, M_{0}^{\theta_{\nu}} \boldsymbol{Y}_{\boldsymbol{h}}^{\mathbf{0}}\right\rangle+\left\langle\boldsymbol{W}_{\boldsymbol{h}}^{\mathbf{0}}, M_{h}^{\theta_{\nu}} \boldsymbol{Y}_{\boldsymbol{h}}^{\mathbf{1}}\right\rangle-\left\langle\boldsymbol{W}_{\boldsymbol{h}}^{\boldsymbol{N}-\mathbf{1}}, M_{0}^{\theta_{\nu}} \boldsymbol{Y}_{\boldsymbol{h}}^{\boldsymbol{N}}\right\rangle=0
$$

Then, using that $\boldsymbol{Y}_{\boldsymbol{h}}^{\boldsymbol{N}}=\boldsymbol{Y}_{\boldsymbol{h}}^{\boldsymbol{N - 1}}=0$ and the definition of $\boldsymbol{F}_{\boldsymbol{h}}^{\boldsymbol{n}}$, we simply obtain

$$
\sum_{n=1}^{N-1}\left(\nu^{2} v_{h}^{n} w_{J}^{n}-\theta_{\nu}\left(v_{h}^{n+1}-2 v_{h}^{n}+v_{h}^{n-1}\right) w_{J}^{n}\right)-\left\langle\boldsymbol{W}_{\boldsymbol{h}}^{\mathbf{1}}, M_{h}^{\theta_{\nu}} \boldsymbol{Y}_{\boldsymbol{h}}^{\mathbf{0}}\right\rangle+\left\langle\boldsymbol{W}_{\boldsymbol{h}}^{\mathbf{0}}, M_{h}^{\theta_{\nu}} \boldsymbol{Y}_{\boldsymbol{h}}^{\mathbf{1}}\right\rangle=0
$$

or equivalently

$$
\begin{aligned}
\sum_{n=0}^{N-1}\left(\nu^{2} v_{h}^{n} w_{J}^{n}+\theta_{\nu}\left(v_{h}^{n+1}-v_{h}^{n}\right)\left(w_{J}^{n+1}-w_{J}^{n}\right)\right)+\theta_{\nu}\left(v_{h}^{1}-v_{h}^{0}\right) w_{J}^{1}-\theta_{\nu}\left(v_{h}^{N}-v_{h}^{N-1}\right) w_{J}^{N} & \\
& -\left\langle\boldsymbol{W}_{\boldsymbol{h}}^{\mathbf{1}}, M_{h}^{\theta_{\nu}} \boldsymbol{Y}_{\boldsymbol{h}}^{\mathbf{0}}\right\rangle+\left\langle\boldsymbol{W}_{\boldsymbol{h}}^{\mathbf{0}}, M_{h}^{\theta_{\nu}} \boldsymbol{Y}_{\boldsymbol{h}}^{\mathbf{1}}\right\rangle=0
\end{aligned}
$$


If we assume that

$$
\theta_{\nu}\left(v_{h}^{1}-v_{h}^{0}\right)=0, \quad \theta_{\nu}\left(v_{h}^{N}-v_{h}^{N-1}\right)=0,
$$

then, according to the relation

$$
\left\langle\boldsymbol{W}_{\boldsymbol{h}}^{\mathbf{1}}, M_{h}^{\theta_{\nu}} \boldsymbol{Y}_{\boldsymbol{h}}^{\mathbf{0}}\right\rangle-\left\langle\boldsymbol{W}_{\boldsymbol{h}}^{\mathbf{0}}, M_{h}^{\theta_{\nu}} \boldsymbol{Y}_{\boldsymbol{h}}^{\mathbf{1}}\right\rangle=\left\langle\boldsymbol{W}_{\boldsymbol{h}}^{\mathbf{1}}-\boldsymbol{W}_{\boldsymbol{h}}^{\mathbf{0}}, M_{h}^{\theta_{\nu}} \frac{\boldsymbol{Y}_{\boldsymbol{h}}^{\mathbf{0}}+\boldsymbol{Y}_{\boldsymbol{h}}^{\mathbf{1}}}{2}\right\rangle-\left\langle\frac{\boldsymbol{W}_{\boldsymbol{h}}^{\mathbf{0}}+\boldsymbol{W}_{\boldsymbol{h}}^{\mathbf{1}}}{2}, M_{h}^{\theta_{\nu}}\left(\boldsymbol{Y}_{\boldsymbol{h}}^{\mathbf{1}}-\boldsymbol{Y}_{\boldsymbol{h}}^{\mathbf{0}}\right)\right\rangle
$$

and using $\nu=\Delta t / h$, the equalities $\left(\boldsymbol{Y}_{\boldsymbol{h}}^{\mathbf{0}}+\boldsymbol{Y}_{\boldsymbol{h}}^{\mathbf{1}}\right) / 2=\boldsymbol{y}_{\mathbf{0} \boldsymbol{h}}$ and $\left(\boldsymbol{Y}_{\boldsymbol{h}}^{\mathbf{1}}-\boldsymbol{Y}_{\boldsymbol{h}}^{\mathbf{0}}\right) / \Delta t=\boldsymbol{y}_{\mathbf{1} \boldsymbol{h}}$, we obtain the following characterization:

Lemma 4.1. Given $T>2 \max \left(1, \nu^{2}\right)$, the system $(21)$ is controllable if, for any $\left(\boldsymbol{y}_{\mathbf{0} \boldsymbol{h}}, \boldsymbol{y}_{\mathbf{1} \boldsymbol{h}}\right) \in \mathbb{R}^{2 J}$, there exists $\left(v_{h}^{n}\right)_{n}$ such that $\Delta t \sum_{n=0}^{N-1}\left(v_{h}^{n}\right)^{2}<\infty, \Delta t \sum_{n=0}^{N-1}\left(\frac{v_{h}^{n+1}-v_{h}^{n}}{\Delta t}\right)^{2}<\infty, \Delta t \sum_{n=0}^{N-1}\left(\frac{v_{h}^{n+1}-2 v_{h}^{n}+v_{h}^{n-1}}{\Delta t^{2}}\right)^{2}<\infty$ which satisfies (98) and

$$
\begin{aligned}
& \Delta t \sum_{n=0}^{N-1}\left(v_{h}^{n} \frac{w_{J}^{n}}{h}+\theta_{\nu} h \frac{v_{h}^{n+1}-v_{h}^{n}}{\Delta t} \frac{w_{J}^{n+1}-w_{J}^{n}}{\Delta t}\right) \\
& \quad-\left(\left(-K_{h}^{-1} M_{0}^{\theta_{\nu}} \boldsymbol{y}_{\mathbf{1} \boldsymbol{h}},\left(M_{1}^{\theta_{\nu}}\right)^{-1} M_{0}^{\theta_{\nu}} \boldsymbol{y}_{\mathbf{0}}\right),\left(\frac{\boldsymbol{W}_{\boldsymbol{h}}^{\mathbf{0}}+\boldsymbol{W}_{\boldsymbol{h}}^{\mathbf{1}}}{2}, \frac{\boldsymbol{W}_{\boldsymbol{h}}^{\mathbf{1}}-\boldsymbol{W}_{\boldsymbol{h}}^{\mathbf{0}}}{\Delta t}\right)\right)_{1}=0
\end{aligned}
$$

for any $\left(\boldsymbol{w}_{\mathbf{0}}, \boldsymbol{w}_{\mathbf{1} \boldsymbol{h}}\right) \in \mathbb{R}^{2 J}, \boldsymbol{W}_{\boldsymbol{h}}^{\boldsymbol{n}}$ being the corresponding solution of (32).

Thanks to this characterization, we are now able to prove Theorem 2.7.

Proof of Theorem 2.7. Suppose that the functional $\mathcal{J}_{h}$ has a minimum which is attained at $\left(\hat{\boldsymbol{w}}_{\mathbf{0} \boldsymbol{h}}, \hat{\boldsymbol{w}}_{\mathbf{1 h}}\right)$. It follows that, for any $\lambda>0$ and $\left(\boldsymbol{w}_{\mathbf{0} \boldsymbol{h}}, \boldsymbol{w}_{\mathbf{1} \boldsymbol{h}}\right)$,

$$
\begin{aligned}
0 \leq \frac{1}{\lambda}\left[\mathcal { J } _ { h } \left(\left(\hat{\boldsymbol{w}}_{\mathbf{0} \boldsymbol{h}}, \hat{\boldsymbol{w}}_{\mathbf{1} \boldsymbol{h}}\right)+\right.\right. & \left.\left.\lambda\left(\boldsymbol{w}_{\mathbf{0} \boldsymbol{h}}, \boldsymbol{w}_{\mathbf{1} \boldsymbol{h}}\right)\right)-\mathcal{J}_{h}\left(\left(\hat{\boldsymbol{w}}_{\mathbf{0} \boldsymbol{h}}, \hat{\boldsymbol{w}}_{\mathbf{1} \boldsymbol{h}}\right)\right)\right]=\Delta t \sum_{n=0}^{N-1}\left(\frac{\hat{w}_{J}^{n}}{h} \frac{w_{J}^{n}}{h}+\rho^{n} \theta_{\nu} \frac{\hat{w}_{J}^{n+1}-\hat{w}_{J}^{n}}{\Delta t} \frac{w_{J}^{n+1}-w_{J}^{n}}{\Delta t}\right) \\
& -\left(\left(-K_{h}^{-1} M_{0}^{\theta_{\nu}} \boldsymbol{y}_{\mathbf{1} \boldsymbol{h}},\left(M_{1}^{\theta_{\nu}}\right)^{-1} M_{0}^{\theta_{\nu}} \boldsymbol{y}_{\mathbf{0} \boldsymbol{h}}\right),\left(\frac{\boldsymbol{W}_{\boldsymbol{h}}^{\mathbf{0}}+\boldsymbol{W}_{\boldsymbol{h}}^{\mathbf{1}}}{2}, \frac{\boldsymbol{W}_{\boldsymbol{h}}^{\mathbf{1}}-\boldsymbol{W}_{\boldsymbol{h}}^{\mathbf{0}}}{\Delta t}\right)\right)_{1}+O(\lambda) . \quad(101)
\end{aligned}
$$

By letting $\lambda$ tend to zero, we obtain that

$$
\begin{array}{r}
0 \leq \Delta t \sum_{n=0}^{N-1}\left(\frac{\hat{w}_{J}^{n}}{h} \frac{w_{J}^{n}}{h}+\rho^{n} \theta_{\nu} \frac{\hat{w}_{J}^{n+1}-\hat{w}_{J}^{n}}{\Delta t} \frac{w_{J}^{n+1}-w_{J}^{n}}{\Delta t}\right) \\
-\left(\left(-K_{h}^{-1} M_{0}^{\theta_{\nu}} \boldsymbol{y}_{\mathbf{1} \boldsymbol{h}},\left(M_{1}^{\theta_{\nu}}\right)^{-1} M_{0}^{\theta_{\nu}} \boldsymbol{y}_{\mathbf{0} \boldsymbol{h}}\right),\left(\frac{\boldsymbol{W}_{\boldsymbol{h}}^{\mathbf{0}}+\boldsymbol{W}_{\boldsymbol{h}}^{\mathbf{1}}}{2}, \frac{\boldsymbol{W}_{\boldsymbol{h}}^{\mathbf{1}}-\boldsymbol{W}_{\boldsymbol{h}}^{\mathbf{0}}}{\Delta t}\right)\right)_{1}
\end{array}
$$

In the same way, taking $\lambda<0$, we finally obtain:

$$
\begin{aligned}
& 0=\Delta t \sum_{n=0}^{N-1}\left(\frac{\hat{w}_{J}^{n}}{h} \frac{w_{J}^{n}}{h}+\rho^{n} \theta_{\nu} \frac{\hat{w}_{J}^{n+1}-\hat{w}_{J}^{n}}{\Delta t} \frac{w_{J}^{n+1}-w_{J}^{n}}{\Delta t}\right) \\
& -\left(\left(-K_{h}^{-1} M_{0}^{\theta_{\nu}} \boldsymbol{y}_{\mathbf{1} \boldsymbol{h}},\left(M_{1}^{\theta_{\nu}}\right)^{-1} M_{0}^{\theta_{\nu}} \boldsymbol{y}_{\mathbf{0}}\right),\left(\frac{\boldsymbol{W}_{\boldsymbol{h}}^{\mathbf{0}}+\boldsymbol{W}_{\boldsymbol{h}}^{\mathbf{1}}}{2}, \frac{\boldsymbol{W}_{\boldsymbol{h}}^{\mathbf{1}}-\boldsymbol{W}_{\boldsymbol{h}}^{\mathbf{0}}}{\Delta t}\right)_{1} .\right.
\end{aligned}
$$


Then, according to the Lemma 4.1, this implies that $v_{h}=\left(v_{h}^{n}\right)_{n}$ is a control if and only if:

$$
\sum_{n=0}^{N-1}\left(\frac{\hat{w}_{J}^{n}}{h} \frac{w_{J}^{n}}{h}+\rho^{n} \theta_{\nu} \frac{\hat{w}_{J}^{n+1}-\hat{w}_{J}^{n}}{\Delta t} \frac{w_{J}^{n+1}-w_{J}^{n}}{\Delta t}\right)=\sum_{n=0}^{N-1}\left(v_{h}^{n} \frac{w_{J}^{n}}{h}+\theta_{\nu} h \frac{v_{h}^{n+1}-v_{h}^{n}}{\Delta t} \frac{w_{J}^{n+1}-w_{J}^{n}}{\Delta t}\right)
$$

and then

$$
\begin{array}{r}
\sum_{n=0}^{N-1}\left(\frac{v_{h}^{n}}{h}-\theta_{\nu} h \frac{v_{h}^{n+1}-2 v_{h}^{n}+v_{h}^{n-1}}{\Delta t^{2}}\right) w_{J}^{n}+\frac{\theta_{\nu} h}{\Delta t^{2}}\left(v_{h}^{N}-v_{h}^{N-1}\right) w_{J}^{N}-\frac{\theta_{\nu} h}{\Delta t^{2}}\left(v_{h}^{1}-v_{h}^{0}\right) w_{J}^{0}= \\
\sum_{n=0}^{N-1}\left(\frac{\hat{w}_{J}^{n}}{h^{2}}-\frac{\theta_{\nu}}{\Delta t}\left[\rho^{n} \frac{\hat{w}_{J}^{n+1}-\hat{w}_{J}^{n}}{\Delta t}-\rho^{n-1} \frac{\hat{w}_{J}^{n}-\hat{w}_{J}^{n-1}}{\Delta t}\right]\right) w_{J}^{n} \\
+\frac{\theta_{\nu}}{\Delta t^{2}}\left(\rho^{N-1}\left(\hat{w}_{J}^{N}-\hat{w}_{J}^{N-1}\right) w_{J}^{N}-\rho^{0}\left(\hat{w}_{J}^{1}-\hat{w}_{J}^{0}\right) w_{J}^{0}\right)
\end{array}
$$

Since $\rho^{0}=\rho^{N-1}=0$ and according to (98), this relation shows that $v_{h}$ defined in Theorem 2.7 is a control for (21). Hence, the proof of Theorem 2.7 is complete if we show that there exists a unique minimizer of the functional $\mathcal{J}_{h}$.

Lemma 4.2. The functional $\mathcal{J}_{h}$ defined by (31) has a unique minimizer.

Proof. Let us first remark that $\mathcal{J}_{h}$ is continuous with respect to the norm $\|.\|_{1}$. We use the conservation of the energy to write that

$$
E_{0}^{\theta, \alpha}=\frac{1}{2}\left\|\frac{\boldsymbol{W}_{\boldsymbol{h}}^{\mathbf{0}}+\boldsymbol{W}_{\boldsymbol{h}}^{\mathbf{1}}}{2}, \frac{\boldsymbol{W}_{\boldsymbol{h}}^{\mathbf{1}}-\boldsymbol{W}_{\boldsymbol{h}}^{\mathbf{0}}}{\Delta t}\right\|_{1}^{2}=\frac{1}{2}\left\|\frac{\boldsymbol{W}_{\boldsymbol{h}}^{\boldsymbol{N}-\mathbf{1}}+\boldsymbol{W}_{\boldsymbol{h}}^{\boldsymbol{N}}}{2}, \frac{\boldsymbol{W}_{\boldsymbol{h}}^{\boldsymbol{N}}-\boldsymbol{W}_{\boldsymbol{h}}^{\boldsymbol{N}-\mathbf{1}}}{\Delta t}\right\|_{1}^{2}=\frac{1}{2}\left\|\left(\boldsymbol{w}_{\mathbf{0 h}}, \boldsymbol{w}_{\mathbf{1} \boldsymbol{h}}\right)\right\|_{1}^{2} .
$$

Then, according to the right part of (57), there exists a constant $C_{2}$ such that

$$
\left|\mathcal{J}\left(\left(\boldsymbol{w}_{\mathbf{0} \boldsymbol{h}}, \boldsymbol{w}_{\mathbf{1} \boldsymbol{h}}\right)\right)\right| \leq\left\|\boldsymbol{w}_{\mathbf{0} \boldsymbol{h}}, \boldsymbol{w}_{\mathbf{1} \boldsymbol{h}}\right\|_{1}\left(C_{2}\left\|\boldsymbol{w}_{\mathbf{0} \boldsymbol{h}}, \boldsymbol{w}_{\mathbf{1} \boldsymbol{h}}\right\|_{1}+\left\|-K_{h}^{-1} M_{0}^{\theta_{\nu}} \boldsymbol{y}_{\mathbf{1} \boldsymbol{h}},\left(M_{1}^{\theta_{\nu}}\right)^{-1} M_{0}^{\theta_{\nu}} \boldsymbol{y}_{\mathbf{0} \boldsymbol{h}}\right\|_{1}\right)
$$

Since $\mathcal{J}_{h}$ is strictly convex, let us show that $\mathcal{J}_{h}$ is coercive, i.e.,

$$
\lim _{\left\|\left(\boldsymbol{w}_{\mathbf{O} \boldsymbol{h}}, \boldsymbol{w}_{\mathbf{1} \boldsymbol{h}}\right)\right\|_{1} \rightarrow \infty} \mathcal{J}_{h}\left(\left(\boldsymbol{w}_{\mathbf{O} \boldsymbol{h}}, \boldsymbol{w}_{\mathbf{1} \boldsymbol{h}}\right)\right)=\infty
$$

This result follows from the uniform observability inequality, i.e., the left part of (57): there exists a positive constant $C_{1}$ such that

$$
\mathcal{J}_{h}\left(\boldsymbol{w}_{\mathbf{0} \boldsymbol{h}}, \boldsymbol{w}_{\mathbf{1} \boldsymbol{h}}\right) \geq\left\|\boldsymbol{w}_{\mathbf{0} \boldsymbol{h}}, \boldsymbol{w}_{\mathbf{1} \boldsymbol{h}}\right\|_{1}\left(C_{1}\left\|\boldsymbol{w}_{\mathbf{0} \boldsymbol{h}}, \boldsymbol{w}_{\mathbf{1} \boldsymbol{h}}\right\|_{1}-\left\|-K_{h}^{-1} M_{0}^{\theta_{\nu}} \boldsymbol{y}_{\mathbf{1} \boldsymbol{h}},\left(M_{1}^{\theta_{\nu}}\right)^{-1} M_{0}^{\theta_{\nu}} \boldsymbol{y}_{\mathbf{0} \boldsymbol{h}}\right\|_{1}\right)
$$

This concludes the proof of Lemma 4.2 and also of Theorem 2.7. 


\subsection{Boundedness of the discrete control}

An important property of the control $v_{h}$ given by Theorem 2.7 is the following uniform boundedness result:

Theorem 4.3. The control $v_{h}$ given by Theorem 2.7 satisfies

$$
\Delta t \sum_{n=0}^{N-1}\left[\left(v_{h}^{n}\right)^{2}+\theta_{\nu} h^{2}\left(\frac{v_{h}^{n+1}-v_{h}^{n}}{\Delta t}\right)^{2}\right] \leq C\left\|-K_{h}^{-1} M_{0}^{\theta_{\nu}} \boldsymbol{y}_{\mathbf{1} \boldsymbol{h}},\left(M_{1}^{\theta_{\nu}}\right)^{-1} M_{0}^{\theta_{\nu}} \boldsymbol{y}_{\mathbf{0} \boldsymbol{h}}\right\|_{1}^{2}
$$

where $C$ is a constant independent of $h$ and $\Delta t$.

Moreover, there exists another constant $C^{\prime}>0$ independent of $h$ and $\Delta t$ such that

$$
\max _{n \in[0, N-1]} h^{2}\left|\frac{v_{h}^{n+1}-v_{h}^{n}}{\Delta t}\right| \leq C^{\prime}\left\|-K_{h}^{-1} M_{0}^{\theta_{\nu}} \boldsymbol{y}_{\mathbf{1} \boldsymbol{h}},\left(M_{1}^{\theta_{\nu}}\right)^{-1} M_{0}^{\theta_{\nu}} \boldsymbol{y}_{\mathbf{0} \boldsymbol{h}}\right\|_{1}
$$

Proof. We multiply (35) by $v_{h}^{n}$ and we obtain,

$$
\begin{aligned}
& \Delta t \sum_{n=0}^{N-1}\left[\left(v_{h}^{n}\right)^{2}+\theta_{\nu} h^{2}\left(\frac{v_{h}^{n+1}-v_{h}^{n}}{\Delta t}\right)^{2}\right]=\Delta t \sum_{n=1}^{N-1}\left(\frac{\hat{w}_{J}^{n}}{h} v_{h}^{n}+\rho^{n} \theta_{\nu} h \frac{\hat{w}_{J}^{n+1}-\hat{w}_{J}^{n}}{\Delta t} \frac{v_{h}^{n+1}-v_{h}^{n}}{\Delta t}\right) \\
& \leq\left[\Delta t \sum_{n=0}^{N-1}\left(\left(v_{h}^{n}\right)^{2}+h^{2} \theta_{\nu} \rho^{n}\left(\frac{v_{h}^{n+1}-v_{h}^{n}}{\Delta t}\right)^{2}\right)\right]^{1 / 2}\left[\Delta t \sum_{n=0}^{N-1}\left(\frac{\left(\hat{w}_{J}^{n}\right)^{2}}{h^{2}}+\theta_{\nu} \rho^{n}\left(\frac{\hat{w}_{J}^{n+1}-\hat{w}_{J}^{n}}{\Delta t}\right)^{2}\right)\right]^{1 / 2} \\
& \quad \leq\left[\Delta t \sum_{n=0}^{N-1}\left(\left(v_{h}^{n}\right)^{2}+h^{2} \theta_{\nu}\left(\frac{v_{h}^{n+1}-v_{h}^{n}}{\Delta t}\right)^{2}\right)\right]^{1 / 2}\left[\Delta t \sum_{n=0}^{N-1}\left(\frac{\left(\hat{w}_{J}^{n}\right)^{2}}{h^{2}}+\theta_{\nu} \rho^{n}\left(\frac{\hat{w}_{J}^{n+1}-\hat{w}_{J}^{n}}{\Delta t}\right)^{2}\right)\right]^{1 / 2} .
\end{aligned}
$$

Hence,

$$
\Delta t \sum_{n=0}^{N-1}\left(\left(v_{h}^{n}\right)^{2}+h^{2} \theta_{\nu}\left(\frac{v_{h}^{n+1}-v_{h}^{n}}{\Delta t}\right)^{2}\right) \leq \Delta t \sum_{n=0}^{N-1}\left(\frac{\left(\hat{w}_{J}^{n}\right)^{2}}{h^{2}}+\theta_{\nu} \rho^{n}\left(\frac{\hat{w}_{J}^{n+1}-\hat{w}_{J}^{n}}{\Delta t}\right)^{2}\right) .
$$

Since $\left(\hat{\boldsymbol{w}}_{\mathbf{0} \boldsymbol{h}}, \hat{\boldsymbol{w}}_{\mathbf{1} \boldsymbol{h}}\right)$ is a minimizer for $\mathcal{J}_{h}$, we have

$$
\mathcal{J}_{h}\left(\left(\hat{\boldsymbol{w}}_{\mathbf{0} \boldsymbol{h}}, \hat{\boldsymbol{w}}_{\mathbf{1} \boldsymbol{h}}\right)\right) \leq \mathcal{J}_{h}((\mathbf{0}, \mathbf{0}))=0
$$

Consequently,

$$
\begin{aligned}
& \Delta t \sum_{n=0}^{N-1}\left[\left(\frac{\hat{w}_{J}^{n}}{h}\right)^{2}+\rho^{n} \theta_{\nu}\left(\frac{\hat{w}_{J}^{n+1}-\hat{w}_{J}^{n}}{\Delta t}\right)^{2}\right] \\
& \quad \leq 2\left(\left(-K_{h}^{-1} M_{0}^{\theta_{\nu}} \boldsymbol{y}_{\mathbf{1} \boldsymbol{h}},\left(M_{1}^{\theta_{\nu}}\right)^{-1} M_{0}^{\theta_{\nu}} \boldsymbol{y}_{\mathbf{0} \boldsymbol{h}}\right),\left(\frac{\boldsymbol{W}_{\boldsymbol{h}}^{\mathbf{0}}+\boldsymbol{W}_{\boldsymbol{h}}^{\mathbf{1}}}{2}, \frac{\boldsymbol{W}_{\boldsymbol{h}}^{\mathbf{1}}-\boldsymbol{W}_{\boldsymbol{h}}^{\mathbf{0}}}{\Delta t}\right)\right)_{1} \\
& \quad \leq 2\left\|-K_{h}^{-1} M_{0}^{\theta_{\nu}} \boldsymbol{y}_{\mathbf{1} \boldsymbol{h}},\left(M_{1}^{\theta_{\nu}}\right)^{-1} M_{0}^{\theta_{\nu}} \boldsymbol{y}_{\mathbf{0} \boldsymbol{h}}\right\|\left\|_{1}\right\| \frac{\boldsymbol{W}_{\boldsymbol{h}}^{\mathbf{0}}+\boldsymbol{W}_{\boldsymbol{h}}^{\mathbf{1}}}{2}, \frac{\boldsymbol{W}_{\boldsymbol{h}}^{\mathbf{1}}-\boldsymbol{W}_{\boldsymbol{h}}^{\mathbf{0}}}{\Delta t} \|_{1} \\
& \quad \leq 2 \sqrt{2 E_{0}^{\theta, \alpha}}\left\|-K_{h}^{-1} M_{0}^{\theta_{\nu}} \boldsymbol{y}_{\mathbf{1} \boldsymbol{h}},\left(M_{1}^{\theta_{\nu}}\right)^{-1} M_{0}^{\theta_{\nu}} \boldsymbol{y}_{\mathbf{0} \boldsymbol{h}}\right\|_{1} \\
& \leq 2 \sqrt{2 C_{2}}\left(\Delta t \sum_{n=0}^{N-1}\left[\left(\frac{\hat{w}_{J}^{n}}{h}\right)^{2}+\rho^{n} \theta_{\nu}\left(\frac{\hat{w}_{J}^{n+1}-\hat{w}_{J}^{n}}{\Delta t}\right)^{2}\right]\right)^{1 / 2}\left\|-K_{h}^{-1} M_{0}^{\theta_{\nu}} \boldsymbol{y}_{\mathbf{1} \boldsymbol{h}},\left(M_{1}^{\theta_{\nu}}\right)^{-1} M_{0}^{\theta_{\nu}} \boldsymbol{y}_{\mathbf{0} \boldsymbol{h}}\right\|_{1}
\end{aligned}
$$


hence

$$
\Delta t \sum_{n=0}^{N-1}\left[\left(\frac{\hat{w}_{J}^{n}}{h}\right)^{2}+\rho^{n} \theta_{\nu}\left(\frac{\hat{w}_{J}^{n+1}-\hat{w}_{J}^{n}}{\Delta t}\right)^{2}\right] \leq 8 C_{2}\left\|-K_{h}^{-1} M_{0}^{\theta_{\nu}} \boldsymbol{y}_{1 \boldsymbol{h}},\left(M_{1}^{\theta_{\nu}}\right)^{-1} M_{0}^{\theta_{\nu}} \boldsymbol{y}_{\mathbf{o}}\right\|_{1}^{2} .
$$

Inequality (110) follows from (113) and (116). We now prove (111). We sum the relation (35) for $n$ from 0 to $p$ leading to

$$
\Delta t \sum_{n=0}^{p} v_{h}^{n}-\theta_{\nu} h^{2} \frac{v_{h}^{p+1}-v_{h}^{p}}{\Delta t}=\Delta t \sum_{n=0}^{p} \frac{\hat{W}_{J}^{n}}{h}-\theta_{\nu} h \rho^{p} \frac{\hat{W}_{J}^{p+1}-\hat{W}_{J}^{p}}{\Delta t}
$$

and then

$$
\begin{aligned}
h^{2}\left|\frac{v_{h}^{p+1}-v_{h}^{p}}{\Delta t}\right| & \leq \Delta t \theta_{\nu}^{-1}\left|\sum_{n=0}^{p} v_{h}^{n}\right|+h \rho^{p}\left|\frac{\hat{W}_{J}^{p+1}-\hat{W}_{J}^{p}}{\Delta t}\right|+\theta_{\nu}^{-1} \Delta t\left|\sum_{n=0}^{p} \frac{\hat{W}_{J}^{n}}{h}\right| \\
& \leq \theta_{\nu}^{-1} \sqrt{T}\left(\Delta t \sum_{n=0}^{N}\left(v_{h}^{n}\right)^{2}\right)^{1 / 2}+\theta_{\nu}^{-1} \sqrt{T}\left(\Delta t \sum_{n=0}^{N}\left(\frac{\hat{W}_{J}^{n}}{h}\right)^{2}\right)^{1 / 2}+h\left|\frac{\hat{W}_{J}^{p+1}-\hat{W}_{J}^{p}}{\Delta t}\right| .
\end{aligned}
$$

The first two terms can be bounded by (110) and (116). Concerning the last one, we observe that it is part of the energy associated to the initial condition $\left(\boldsymbol{w}_{\mathbf{0} \boldsymbol{h}}, \boldsymbol{w}_{\mathbf{1} \boldsymbol{h}}\right)$, and we easily obtain that

$$
h^{2}\left|\frac{\hat{W}_{J}^{p+1}-\hat{W}_{J}^{p}}{\Delta t}\right|^{2} \leq 4 h E_{0}^{\theta, \alpha}<4 E_{0}^{\theta, \alpha}
$$

We conclude using that $E_{0}^{\theta, \alpha} \leq C\left\|-K_{h}^{-1} M_{0}^{\theta_{\nu}} \boldsymbol{y}_{1 \boldsymbol{h}},\left(M_{1}^{\theta_{\nu}}\right)^{-1} M_{0}^{\theta_{\nu}} \boldsymbol{y}_{\mathbf{o h}}\right\|_{1}^{2}$.

Definition 4.4. Let $(\boldsymbol{U}, \boldsymbol{V}) \in \mathbb{R}^{2 J}$ and $(\tilde{\boldsymbol{U}}, \tilde{\boldsymbol{V}}) \in \mathbb{R}^{2 J}$. We then define the following scalar product

$$
((\boldsymbol{U}, \boldsymbol{V}),(\tilde{\boldsymbol{U}}, \tilde{\boldsymbol{V}}))_{-1}=\left(\left(-K_{h}^{-1} M_{0}^{\theta_{\nu}} \boldsymbol{V},\left(M_{1}^{\theta_{\nu}}\right)^{-1} M_{0}^{\theta_{\nu}} \boldsymbol{U}\right),\left(-K_{h}^{-1} M_{0}^{\theta_{\nu}} \tilde{\boldsymbol{V}},\left(M_{1}^{\theta_{\nu}}\right)^{-1} M_{0}^{\theta_{\nu}} \tilde{\boldsymbol{U}}\right)\right)_{1}
$$

and

$$
\|\boldsymbol{U}, \boldsymbol{V}\|_{-1}^{2}=\left\|-K_{h}^{-1} M_{0}^{\theta_{\nu}} \boldsymbol{V},\left(M_{1}^{\theta_{\nu}}\right)^{-1} M_{0}^{\theta_{\nu}} \boldsymbol{U}\right\|_{1}^{2}=\left(M_{0}^{\theta_{\nu}} \boldsymbol{V}, K_{h}^{-1} M_{0}^{\theta_{\nu}} \boldsymbol{V}\right)+\left(M_{0}^{\theta_{\nu}} \boldsymbol{U},\left(M_{1}^{\theta_{\nu}}\right)^{-1} M_{0}^{\theta_{\nu}} \boldsymbol{U}\right) .
$$

Remark 4.5. $(,)_{-1}$ is an inner product and $\|\cdot\|_{-1}$ is a norm on $\mathbb{R}^{2 J}$. Actually, this norm is equivalent to the norm $\|\cdot\|_{l^{2} \times h^{-1}}$. For $(\theta, \alpha, \nu) \in \mathcal{C}$, we obtain

$$
\left\|\boldsymbol{y}_{\mathbf{0} \boldsymbol{h}}, \boldsymbol{y}_{\mathbf{1} \boldsymbol{h}}\right\|_{-1}^{2}=\frac{1}{2} \sum_{k=1}^{J}\left(\cos ^{2}\left(\frac{k \pi h}{2}\right)+\nu^{2} \sin ^{2}\left(\frac{k \pi h}{2}\right)\right)\left(\left(\bar{a}_{k, h}\right)^{2}+\left(\frac{\bar{b}_{k, h}}{\mu_{k, h}}\right)^{2}\right) .
$$

The proof is presented in the subsection 7.1. On the other hand, from the left part of the observability inequality (57), from (106) and (110), we deduce that

$$
\left\|\hat{\boldsymbol{w}}_{\mathbf{0} \boldsymbol{h}}, \hat{\boldsymbol{w}}_{1 \boldsymbol{h}}\right\|_{1}^{2} \leq 2 E_{N}^{\theta, \alpha} \leq C\left\|\boldsymbol{y}_{\mathbf{0} \boldsymbol{h}}, \boldsymbol{y}_{1 \boldsymbol{h}}\right\|_{-1}^{2} .
$$

Thus, the sequence of minimizers $\left(\hat{\boldsymbol{w}}_{\mathbf{0} \boldsymbol{h}}, \hat{\boldsymbol{w}}_{\mathbf{1} \boldsymbol{h}}\right)_{h>0}$ is bounded in the $\|\cdot\|_{1}$-norm if the same property holds for $\left(\boldsymbol{y}_{\mathbf{0} \boldsymbol{h}}, \boldsymbol{y}_{\mathbf{1} \boldsymbol{h}}\right)_{h>0}$ in the $\|\cdot\|_{-1}$-norm. 


\subsection{Convergence of the discrete controls. Proof of Theorem 2.8}

Let us now show the convergence of the controls $v_{h}$ of the discrete system (17) to the HUM control of the continuous system (1). In order to obtain this result, we use the Fourier decompositions (11) and (29) of the initial data.

Proof of Theorem 2.8.

1. Proof of the weak convergence.

For every $h$ and $\Delta t$, let $v_{h}$ be the control given by Theorem 2.7 and $\left(\hat{\boldsymbol{w}}_{\mathbf{0} \boldsymbol{h}}, \hat{\boldsymbol{w}}_{\mathbf{1} \boldsymbol{h}}\right)$ be the minimizer of the functional $\mathcal{J}_{h}$. From (37), it follows that the sequence $\left(\boldsymbol{y}_{\mathbf{0} \boldsymbol{h}}, \boldsymbol{y}_{\mathbf{1} \boldsymbol{h}}\right)_{h}$ is uniformly bounded in $l^{2} \times h^{-1}$, i.e. $\sum_{k>0}\left(\bar{a}_{k, h}\right)^{2}+\left(\bar{b}_{k, h} /\left(\mu_{k, h}\right)\right)^{2}<\infty$ and consequently, according to (122), also uniformly bounded in the $\|\cdot\|_{-1}$ norm. Therefore, Theorem 4.3 implies that the sequences

$$
\begin{aligned}
& \left\|Q\left(v_{h}\right)\right\|_{L^{2}(0, T)}^{2}=\Delta t \sum_{n=0}^{N-1}\left(v_{h}^{n}\right)^{2}, \quad h^{2}\left\|P^{\prime}\left(v_{h}\right)\right\|_{L^{2}(0, T)}^{2}=h^{2} \Delta t \sum_{n=0}^{N-1}\left(\frac{v_{h}^{n+1}-v_{h}^{n}}{\Delta t}\right)^{2}, \\
& h^{2}\left\|P^{\prime}\left(v_{h}\right)\right\|_{L^{\infty}(0, T)}=h^{2} \max _{n \in[0, N-1]}\left|\frac{v_{h}^{n+1}-v_{h}^{n}}{\Delta t}\right|
\end{aligned}
$$

are uniformly bounded. This is equivalent to say that $Q\left(v_{h}\right), \theta_{\nu} h P^{\prime}\left(v_{h}\right)$ are uniformly bounded in $L^{2}(0, T)$ and that $\theta_{\nu} h^{2} P^{\prime}\left(v_{h}\right)$ is uniformly bounded in $L^{\infty}(0, T)$. Hence, there exists a subsequence (denoted in the same way) and $v, \tilde{v} \in L^{2}(0, T)$ such that

$$
Q\left(v_{h}\right) \rightarrow v, \quad \theta_{\nu} h P^{\prime}\left(v_{h}\right) \rightarrow \tilde{v} \quad \text { in } L^{2}(0, T)
$$

To show that $\tilde{v}=0$ (in the case $\left.\theta_{\nu} \neq 0\right)$, we compute that

$$
\begin{aligned}
\left\|P\left(v_{h}\right)\right\|_{L^{2}(0, T)}^{2} & =\frac{\Delta t}{3} \sum_{n=0}^{N-1}\left(\left(v_{h}^{n}\right)^{2}+v_{h}^{n} v_{h}^{n+1}+\left(v_{h}^{n+1}\right)^{2}\right) \\
& \leq \frac{\Delta t}{2} \sum_{n=0}^{N-1}\left(\left(v_{h}^{n}\right)^{2}+\left(v_{h}^{n+1}\right)^{2}\right)=\frac{\Delta t}{2} \sum_{n=0}^{N-1}\left(v_{h}^{n}\right)^{2}+\frac{\Delta t}{2} \sum_{n=1}^{N}\left(v_{h}^{n}\right)^{2} \\
& \leq\left\|Q\left(v_{h}\right)\right\|_{L^{2}(0, T)}^{2}+\Delta t\left(v_{h}^{N}\right)^{2}=\left\|Q\left(v_{h}\right)\right\|_{L^{2}(0, T)}^{2}+\Delta t\left(v_{h}^{N-1}\right)^{2} \leq 2\left\|Q\left(v_{h}\right)\right\|_{L^{2}(0, T)}^{2},
\end{aligned}
$$

where we used $v_{h}^{N-1}=v_{h}^{N}$ from (98) in the case $\theta_{\nu} \neq 0$. This implies that the sequence $\left(h P\left(v_{h}\right)\right)_{h}$ is bounded in $H^{1}(0, T)$. Hence, there exist $v_{1}$ and $\hat{v}$ such that

$$
\theta_{\nu} P\left(v_{h}\right) \rightarrow v_{1}, \quad \theta_{\nu} h P\left(v_{h}\right) \rightarrow \hat{v}, \quad \theta_{\nu} h P^{\prime}\left(v_{h}\right) \rightarrow \tilde{v}^{\prime} \quad \text { in } L^{2}([0, T])
$$

It follows that $\hat{v}=0$ and therefore $\tilde{v}=0$. The last convergence in Theorem 2.8 is a consequence of the bound of $\theta_{\nu} h^{2} P^{\prime}\left(v_{h}\right)$ in $L^{\infty}(0, T)$ and the fact that its weak limit in $L^{2}(0, T)$ is equal to 0 .

2. Identification of the limit. We show that $v$ is the HUM control of the continuous system (1). In this respect, it is necessary and sufficient to verify the assumptions from Remark 1.3.

To prove the first assumption (1.), let us first remark that it is sufficient to show that (7) is satisfied by the eigenfunctions $\phi_{k}$ of the wave operator, for all $k>0$. Indeed, from the continuity of the linear form $\Lambda: H_{0}^{1}(0,1) \times L^{2}(0,1) \rightarrow \mathbb{R}$

$$
\Lambda\left(w_{0}, w_{1}\right)=\int_{0}^{T} v(t) w_{x}(1, t) \mathrm{d} t-\left\langle y_{1}, w(., 0)\right\rangle_{H^{-1}, H_{0}^{1}}+\int_{0}^{1} y_{0}(x) w^{\prime}(x, 0) \mathrm{d} x .
$$


It follows that $(7)$ holds for any $\left(w_{0}, w_{1}\right) \in H_{0}^{1}(0,1) \times L^{2}(0,1)$ if and only if it is verified on a basis of the space $H_{0}^{1}(0,1) \times L^{2}(0,1)$. We then first consider $w$ of the form

$$
w(., t)=\cos (p \pi t) \phi_{p} .
$$

Then, we obtain that $v$ is a control for the system (1) if and only if

$$
\int_{0}^{T} v(t) \cos (p \pi t) \mathrm{d} t=\frac{1}{2 p \pi} a_{p}, \quad \forall p>0 .
$$

Furthermore, we remark that the characterization of Lemma 4.1 of the discrete control $v_{h}$ of system (21) takes the form

$$
\begin{aligned}
\int_{0}^{T}\left(\frac{1}{h} Q\left(v_{h}\right) Q\left(w_{J}\right)+\theta_{\nu}\right. & \left.h P^{\prime}\left(v_{h}\right) P^{\prime}\left(w_{J}\right)\right) \mathrm{d} t \\
& -\left(\left(-K_{h}^{-1} M_{0}^{\theta_{\nu}} \boldsymbol{y}_{\mathbf{1} \boldsymbol{h}},\left(M_{1}^{\theta_{\nu}}\right)^{-1} M_{0}^{\theta_{\nu}} \boldsymbol{y}_{\mathbf{0}}\right),\left(\frac{\boldsymbol{W}_{\boldsymbol{h}}^{\mathbf{0}}+\boldsymbol{W}_{\boldsymbol{h}}^{\mathbf{1}}}{2}, \frac{\boldsymbol{W}_{\boldsymbol{h}}^{\mathbf{1}}-\boldsymbol{W}_{\boldsymbol{h}}^{\mathbf{0}}}{\Delta t}\right)\right)_{1}=0
\end{aligned}
$$

for any $\left(\boldsymbol{w}_{\mathbf{0}}, \boldsymbol{w}_{\mathbf{1} \boldsymbol{h}}\right) \in \mathbb{R}^{2 J}$. Using the definition of the norm $\|.\|_{1}$ and $\boldsymbol{y}_{\mathbf{h}}$ and $\boldsymbol{y}_{\mathbf{1} \boldsymbol{h}}$ (see (21)), we simply have

$$
\begin{aligned}
& \left(\left(-K_{h}^{-1} M_{0}^{\theta_{\nu}} \boldsymbol{y}_{\mathbf{1} \boldsymbol{h}},\left(M_{1}^{\theta_{\nu}}\right)^{-1} M_{0}^{\theta_{\nu}} \boldsymbol{y}_{\mathbf{0}}\right),\left(\frac{\boldsymbol{W}_{\boldsymbol{h}}^{\mathbf{0}}+\boldsymbol{W}_{\boldsymbol{h}}^{\mathbf{1}}}{2}, \frac{\boldsymbol{W}_{\boldsymbol{h}}^{\mathbf{1}}-\boldsymbol{W}_{\boldsymbol{h}}^{\mathbf{0}}}{\Delta t}\right)\right)_{1} \\
& =\left(M_{0}^{\theta_{\nu}} \boldsymbol{y}_{\mathbf{1} \boldsymbol{h}}, \frac{\boldsymbol{W}_{\boldsymbol{h}}^{\mathbf{0}}+\boldsymbol{W}_{\boldsymbol{h}}^{\mathbf{1}}}{2}\right)+\left(M_{0}^{\theta_{\nu}} \boldsymbol{y}_{\mathbf{0} \boldsymbol{h}}, \frac{\boldsymbol{W}_{\boldsymbol{h}}^{\mathbf{1}}-\boldsymbol{W}_{\boldsymbol{h}}^{\mathbf{0}}}{\Delta t}\right)=\frac{1}{\Delta t}\left(\left(M_{0}^{\theta_{\nu}} \boldsymbol{Y}_{\boldsymbol{h}}^{\mathbf{1}}, \boldsymbol{W}_{\boldsymbol{h}}^{\mathbf{1}}\right)-\left(M_{0}^{\theta_{\nu}} \boldsymbol{Y}_{\boldsymbol{h}}^{\mathbf{0}}, \boldsymbol{W}_{\boldsymbol{h}}^{\mathbf{0}}\right)\right) .
\end{aligned}
$$

According to (129), we consider

$$
\boldsymbol{W}_{\boldsymbol{h}}^{\boldsymbol{n}}=\cos \left(\mu_{p} n \Delta t\right) \phi_{\boldsymbol{h}}^{\boldsymbol{p}}
$$

Then, using the fact that $\mu_{k} \rightarrow k \pi$ when $h \rightarrow 0$, for all $k>0$, similar computations than before show that

$$
\lim _{h \rightarrow 0} \frac{1}{\Delta t}\left(\left(M_{0}^{\theta_{\nu}} \boldsymbol{Y}_{\boldsymbol{h}}^{\mathbf{1}}, \boldsymbol{W}_{\boldsymbol{h}}^{\mathbf{1}}\right)-\left(M_{0}^{\theta_{\nu}} \boldsymbol{Y}_{\boldsymbol{h}}^{\mathbf{0}}, \boldsymbol{W}_{\boldsymbol{h}}^{\mathbf{0}}\right)\right)=-\frac{1}{2} a_{p}
$$

Moreover, we have $w_{J}^{n}=\cos \left(\mu_{p} n \Delta t\right) \sin (J p \pi h)=-\cos \left(\mu_{p} t_{n}\right) \sin (p \pi h)$. This implies that

$$
\int_{0}^{T} \frac{1}{h} Q\left(v_{h}\right)(t) Q\left(w_{J}\right)(t) \mathrm{d} t=-\int_{0}^{T} Q\left(v_{h}\right) \cos \left(\mu_{p} t_{n}\right) \frac{\sin (p \pi h)}{h} \mathbb{1}_{t \in\left[t_{n}, t_{n+1}\right]} \mathrm{d} t,
$$

and taking into account that

$$
Q\left(v_{h}\right) \rightarrow v, \cos \left(\mu_{p} t_{n}\right) \mathbb{1}_{t \in\left[t_{n}, t_{n+1}\right]} \rightarrow \cos (p \pi t) \text { in } L^{2}(0, T),
$$

we obtain

$$
\lim _{h \rightarrow 0} \int_{0}^{T} \frac{1}{h} Q\left(v_{h}\right)(t) Q\left(w_{J}\right)(t) \mathrm{d} t=\int_{0}^{T} v(t) \cos (p \pi t) p \pi \mathrm{d} t .
$$

In addition, we have by definition of the extension $P$ :

$$
P\left(w_{J}\right)(t)=-\frac{\sin (p \pi h)}{\Delta t}\left[\left(t-t_{n}\right) \cos \left(\mu_{p}(n+1) \Delta t\right)+\left(t_{n+1}-t\right) \cos \left(\mu_{p} n \Delta t\right)\right] \mathbb{1}_{t \in\left[t_{n}, t_{n+1}\right]},
$$


leading to $\left(P\left(w_{J}\right)\right)^{\prime}(t)=-\frac{\sin (p \pi h)}{\Delta t}\left[\cos \left(\mu_{p}(n+1) \Delta t\right)-\cos \left(\mu_{p} n \Delta t\right)\right] \mathbb{1}_{t \in\left[t_{n}, t_{n+1}\right]}$. Then, using that $\frac{\sin p \pi h}{\Delta t}=$ $\frac{1}{\nu} \frac{\sin p \pi h}{h} \leq \frac{p \pi}{\nu}$ and the weak convergence $h P^{\prime}\left(v_{h}\right) \rightarrow 0$ in $L^{2}(0, T)$, we have that

$$
\lim _{h \rightarrow 0} \int_{0}^{T} h P^{\prime}\left(v_{h}\right)(t) P^{\prime}\left(w_{J}\right) \mathrm{d} t=0
$$

It follows that the limit $v$ satisfies (130). We obtain the same result if we consider $w$ of the form $w(., t)=$ $\sin (p \pi t) \phi_{p}$ instead of (129). Therefore, $v$ is a control for the system (1) and the first part of Remark 1.3 is proved.

Let us now prove the second part. Let $\left(\hat{\boldsymbol{w}}_{\mathbf{0} \boldsymbol{h}}, \hat{\boldsymbol{w}}_{\mathbf{1} \boldsymbol{h}}\right)$ be the minimizer of the function $\mathcal{J}_{h}$. From Remark 4.5 and the boundedness of $\left(\boldsymbol{y}_{\mathbf{0}}, \boldsymbol{y}_{\mathbf{1} \boldsymbol{h}}\right)$, we deduce that the sequence $\left(a_{k, h}, b_{k, h} / \mu_{k, h}\right)_{k}$, associated to $\boldsymbol{W}_{\boldsymbol{h}}^{\boldsymbol{n}}$, is uniformly bounded in $l^{2}$. Therefore, there exists a subsequence, denoted in the same way, which converges weakly to $\left(a_{k}, b_{k} /(k \pi)\right)_{k}$. Thus, by Lemma 3.16, the sequence $\left(Q\left(\hat{w}_{J} / h\right)\right)_{h}$ tends weakly to $\left(\hat{w}_{x}(1,).\right)$ in $L^{2}(0, T)$, where $\hat{w}$ is solution of $(6)$ with initial condition $\left(\hat{w}_{0}, \hat{w}_{1}\right)$. Let $\psi$ be a test function in $H^{1}(0, T)$. We have that

$$
\begin{aligned}
\int_{0}^{T} v(t) \psi(t) \mathrm{d} t & =\lim _{h \rightarrow 0} \int_{0}^{T}\left(Q\left(v_{h}\right)(t) Q(\psi)(t) \mathrm{d} t+\theta_{\nu} h^{2} P^{\prime}\left(v_{h}\right) P^{\prime}(\psi)(t)\right) \mathrm{d} t \\
& =\lim _{h \rightarrow 0} \Delta t \sum_{n=0}^{N-1}\left(v_{h}^{n} \psi\left(t_{n}\right)+\theta_{\nu} h^{2} \frac{v_{h}^{n+1}-v_{h}^{n}}{\Delta t} \frac{\psi\left(t_{n+1}\right)-\psi\left(t_{n}\right)}{\Delta t}\right) .
\end{aligned}
$$

Then, from (104), we obtain

$$
\begin{aligned}
\int_{0}^{T} v(t) \psi(t) \mathrm{d} t & =\lim _{h \rightarrow 0} \Delta t \sum_{n=0}^{N-1}\left(\frac{\hat{w}_{h}^{n}}{h} \psi\left(t_{n}\right)+\theta_{\nu} h \rho^{n} \frac{\hat{w}_{h}^{n+1}-\hat{w}_{h}^{n}}{\Delta t} \frac{\psi\left(t_{n+1}\right)-\psi\left(t_{n}\right)}{\Delta t}\right) \\
& =\lim _{h \rightarrow 0} \int_{0}^{T}\left(Q\left(\frac{\hat{w}_{J}}{h}\right)(t) Q(\psi)(t) \mathrm{d} t+\theta_{\nu} h \rho(t) P^{\prime}\left(\hat{w}_{h}\right) P^{\prime}(\psi)(t)\right) \mathrm{d} t
\end{aligned}
$$

Let us note that from (116) and Remark 4.5, that

$$
h^{2} \Delta t \sum_{n=0}^{N-1}\left(\rho^{n}\right)^{2}\left(\frac{\hat{w}_{J}^{n+1}-\hat{w}_{J}^{n}}{\Delta t}\right)^{2} \leq h^{2} \Delta t \sum_{n=0}^{N-1} \rho^{n}\left(\frac{\hat{w}_{J}^{n+1}-\hat{w}_{J}^{n}}{\Delta t}\right)^{2} \leq C h^{2}\left\|\boldsymbol{y}_{\mathbf{0} \boldsymbol{h}}, \boldsymbol{y}_{\mathbf{1} \boldsymbol{h}}\right\|_{-1}^{2}
$$

This implies that $\lim _{h \rightarrow 0} h \rho P^{\prime}\left(\hat{w}_{J}\right)=0$ in $L^{2}([0, T])$. We finally obtain that

$$
\int_{0}^{T} v(t) \psi(t) \mathrm{d} t=\int_{0}^{T} \hat{w}_{x}(1, t) \psi(t) \mathrm{d} t, \quad \forall \psi \in H^{1}(0,1)
$$

which implies that $v(t)=\hat{w}_{x}(1, t)$. This concludes the proof of the weak converge of the sequence $\left(v_{h}\right)_{h}$.

\section{Proof of the strong convergence}

We have already proved that $\left(v_{h}\right)_{h>0}$ converges weakly to $v=\hat{w}_{x}(1,$.$) in L^{2}(0, T)$ when $h$ goes to zero, where $\hat{w}$ is solution of $(6)$ with initial conditions $\left(\hat{w}_{0}, \hat{w}_{1}\right)$, the minimizer of $\mathcal{J}$. By considering in (100) the test function $\left(\hat{\boldsymbol{w}}_{\mathbf{0} \boldsymbol{h}}, \hat{\boldsymbol{w}}_{\mathbf{1} \boldsymbol{h}}\right)$, we obtain from (104) that

$$
\Delta t \sum_{n=1}^{N-1}\left(\left(\frac{\hat{w}_{J}^{n}}{h}\right)^{2}+\rho^{n} \theta_{\nu}\left(\frac{\hat{w}_{J}^{n+1}-\hat{w}_{J}^{n}}{\Delta t}\right)^{2}\right)=\frac{1}{\Delta t}\left(\left(M_{0}^{\theta_{\nu}} \boldsymbol{Y}_{\boldsymbol{h}}^{\mathbf{1}}, \hat{\boldsymbol{W}}_{\boldsymbol{h}}^{\mathbf{1}}\right)-\left(M_{0}^{\theta_{\nu}} \boldsymbol{Y}_{\boldsymbol{h}}^{\mathbf{0}}, \hat{\boldsymbol{W}}_{\boldsymbol{h}}^{\mathbf{0}}\right)\right)
$$


Now, from the strong convergence of the Fourier coefficient, a computation as performed for the weak convergence leads to

$$
\lim _{h \rightarrow 0} \frac{1}{\Delta t}\left(\left(M_{0}^{\theta_{\nu}} \boldsymbol{Y}_{\boldsymbol{h}}^{\mathbf{1}}, \boldsymbol{W}_{\boldsymbol{h}}^{\mathbf{1}}\right)-\left(M_{0}^{\theta_{\nu}} \boldsymbol{Y}_{\boldsymbol{h}}^{\mathbf{0}}, \boldsymbol{W}_{\boldsymbol{h}}^{\mathbf{0}}\right)\right)=\left\langle u_{1}, \hat{w}(., 0)\right\rangle_{H^{-1}, H_{0}^{1}}-\int_{0}^{1} u_{0}(x) \hat{w}^{\prime}(x, 0) \mathrm{d} x .
$$

It follows from (144) that

$$
\lim _{h \rightarrow 0} \int_{0}^{T}\left(\left(Q\left(\frac{\hat{w}_{J}}{h}\right)\right)^{2}+\theta_{\nu} \rho(t)\left(P^{\prime}\left(\hat{w}_{J}\right)\right)^{2}\right) \mathrm{d} t=\int_{0}^{T}\left(\hat{w}_{x}\right)^{2}(1, t) \mathrm{d} t .
$$

Now, from the weak convergence of $Q\left(\hat{w}_{J} / h\right)$ to $\hat{w}_{x}(1,$.$) proved in Lemma 3.16, we note that$

$$
\begin{aligned}
\int_{0}^{T}\left(\hat{w}_{x}\right)^{2}(1, t) \mathrm{d} t & \leq \lim _{h \rightarrow 0} \inf \int_{0}^{T}\left(Q\left(\frac{\hat{w}_{J}}{h}\right)\right)^{2} \mathrm{~d} t \leq \lim _{h \rightarrow 0} \sup \int_{0}^{T}\left(Q\left(\frac{\hat{w}_{J}}{h}\right)\right)^{2} \mathrm{~d} t \\
& \leq \lim _{h \rightarrow 0} \sup \int_{0}^{T}\left(\left(Q\left(\frac{\hat{w}_{J}}{h}\right)\right)^{2}+\theta_{\nu} \rho(t)\left(P^{\prime}\left(\hat{w}_{J}\right)\right)^{2}\right) \mathrm{d} t=\int_{0}^{T}\left(\hat{w}_{x}\right)^{2}(1, t) \mathrm{d} t .
\end{aligned}
$$

We conclude that $Q\left(\hat{w}_{J} / h\right)$ converges strongly in $L^{2}(0, T)$ to $\hat{w}_{x}(1,$.$) when h$ tends to zero. In addition, from (146), we deduce that

$$
\lim _{h \rightarrow 0} \int_{0}^{T} \rho(t)\left(P^{\prime}\left(\left(\hat{w}_{J}\right)\right)\right)^{2} \mathrm{~d} t=0 .
$$

Now, since (see the first equality in (112)),

$$
\int_{0}^{T}\left(\left(Q\left(v_{h}\right)\right)^{2}(t)+\theta_{\nu}\left(h P^{\prime}\left(v_{h}\right)\right)^{2}(t)\right) \mathrm{d} t=\int_{0}^{T}\left(Q\left(\frac{\hat{w}_{J}}{h}\right) Q\left(v_{h}\right)+h \theta_{\nu} \rho(t) P^{\prime}\left(\hat{w}_{J}\right)(t) P^{\prime}\left(v_{h}\right)\right) \mathrm{d} t
$$

and if we take into account the former strong convergence results and the weak convergence of $Q\left(v_{h}\right)$ and $h P^{\prime}\left(v_{h}\right)$, it follows that

$$
\lim _{h \rightarrow 0} \int_{0}^{T}\left(\left(Q\left(v_{h}\right)\right)^{2}(t)+\theta_{\nu}\left(h P^{\prime}\left(v_{h}\right)\right)^{2}(t)\right) \mathrm{d} t=\int_{0}^{T} v(t)^{2} \mathrm{~d} t .
$$

On the other hand,

$$
\begin{aligned}
\int_{0}^{T}\left(Q\left(v_{h}\right)(t)-v(t)\right)^{2} \mathrm{~d} t & \leq \int_{0}^{T}\left(Q\left(v_{h}\right)(t)-v(t)\right)^{2} \mathrm{~d} t+\theta_{\nu} \int_{0}^{T}\left(h P^{\prime}\left(v_{h}\right)\right)^{2}(t) \mathrm{d} t \\
& \leq \int_{0}^{T}\left(\left(Q\left(v_{h}\right)\right)^{2}(t)+\theta_{\nu}\left(h P^{\prime}\left(v_{h}\right)\right)^{2}(t)\right) \mathrm{d} t+\int_{0}^{T} v(t)^{2} \mathrm{~d} t-2 \int_{0}^{T} Q\left(v_{h}\right)(t) v(t) \mathrm{d} t
\end{aligned}
$$

By passing to the limit, it follows that $Q\left(v_{h}\right)$ converges strongly in $L^{2}(0, T)$ to $v(t)$, and next, according to (150), that $h P^{\prime}\left(v_{h}\right)$ converges strongly in $L^{2}(0, T)$ to zero.

To finish the proof of Theorem 2.8, let us show that $h^{2} P^{\prime}\left(v_{h}\right)$ converges strongly to zero in $L^{\infty}(0, T)$. Equality (117) implies

$$
\begin{aligned}
h^{2}\left|\frac{v_{h}^{p+1}-v_{h}^{p}}{\Delta t}\right| & =\left|h \rho^{p} \frac{\hat{w}_{J}^{p+1}-\hat{w}_{J}^{p}}{\Delta t}+\theta_{\nu}^{-1} \int_{0}^{t} Q\left(v_{h}\right)(s) \mathrm{d} s-\theta_{\nu}^{-1} \int_{0}^{t} Q\left(\frac{\hat{w}_{J}}{h}\right) \mathrm{d} s\right| \\
& \leq\left|h \frac{\hat{w}_{J}^{p+1}-\hat{w}_{J}^{p}}{\Delta t}\right|+\theta_{\nu}^{-1} \sqrt{T}\left(\left\|Q\left(\frac{\hat{w}_{J}}{h}\right)-v||_{L^{2}(0, T)}+\right\| Q\left(v_{h}\right)-\left.v\right|_{L^{2}(0, T)}\right) .
\end{aligned}
$$


From (119) and the strong convergence of both $Q\left(\hat{w}_{J} / h\right)$ and $v_{h}$ to the limit control $v$, we conclude that

$$
h^{2}|| P^{\prime}\left(v_{h}\right) \|_{L^{\infty}(0, T)}=h^{2} \max _{p \in[1, N]}\left|\frac{v_{h}^{p+1}-v_{h}^{p}}{\Delta t}\right| \rightarrow 0 \quad \text { when } \quad h \rightarrow 0 .
$$

Remark 4.6. In Theorem 2.8, we assume the convergence of the sequence of the Fourier coefficients of the discrete initial data to the sequence of the Fourier coefficients of the continuous initial data. It is shown in [4] that the weak (resp. strong) convergence in $L^{2}(0,1)$ is fulfilled if the initial data are piecewise continuous (resp. once differentiable with continuous derivative in $[0,1])$ functions.

\subsection{Summary - Invariance of the parametrized family $\left(\mathcal{S}_{h, \Delta t}^{\theta, \alpha}\right)$}

Before the section devoted to numerical simulations, let us summarize the results obtained. The introduction of two parameters $\alpha, \theta$ in the usual centered finite difference scheme permits to restore the commuting property between HUM controllability and numerical discretization. $\theta$ acts on the uniform controllability (and therefore on the convergence of $v_{h}$ ) whereas $\alpha$ acts on the stability of the scheme (and therefore on the convergence of $y_{h}$ ). A natural question that appears concerns the choice of the best parameters. The consistency of the scheme suggests that $\alpha=1 / 12$ or $\theta=1 / 12$ are good candidates. However, quite surprisingly, the two parameters can be eliminated. For all $(\theta, \alpha, \nu) \in \mathcal{C}$, the parameter $\theta_{\nu}$ is invariant with respect to $\alpha$ and $\theta$ :

$$
\theta_{\nu}=\frac{1}{4}\left(1-\nu^{2}\right)
$$

Therefore, the main equation of $\left(\mathcal{S}_{h, \Delta t}^{\theta, \alpha}\right)$ becomes

$$
\Delta_{\Delta t} y_{j}^{n}+\frac{1}{4}\left(h^{2}-\Delta t^{2}\right) \Delta_{h} \Delta_{\Delta t} y_{j}^{n}=\Delta_{h} y_{j}^{n},
$$

and the condition of stability and uniform controllability is

$$
\Delta t<h \sqrt{\frac{T}{2}},
$$

depending only on the time of exact controllability $T>2$. In particular, for $\Delta t=h$, the additional term vanishes and we reobtain the uniform controllability of the usual centered scheme. The additional term defined on the whole space interval $(0,1)$ is neglectable for the small frequency solutions and of order of the energy for the highest frequency solutions. This term permits to damp out the spurious high frequency solutions in uniform time while preserving the convergence towards the solution. For $\Delta t \neq h$, the consistency of this new scheme is of order two in space and time. This kind of method is similar to the Tychonov regularization used in $[8]$ and also more recently revisited in [15].

\section{NumERICAL SIMULATIONS}

The aim of this section is to present some simple numerical experiments in order to confirm the theoretical results that indicate the efficiency of the scheme $\left(\mathcal{S}_{h, \Delta t}^{\theta, \alpha}\right)$ in order to restore uniform controllability. The computations presented in the sequel are performed using the MATLAB package with double precision.

We applied the HUM method to obtain the minimal $L^{2}$-norm control. Let us recall that the HUM method consists of finding the initial conditions $\left(\hat{w}_{0}, \hat{w}_{1}\right)$ of the homogeneous adjoint system (6) minimizing the functional $\mathcal{J}$ defined by (5). The control is then given by $v(t)=\hat{w}_{x}(1, t)$. This linear minimization problem can be reformulated by introducing the operator $\Lambda:\left(H_{0}^{1}(0,1) \times L^{2}(0,1)\right) \rightarrow H^{-1}(0,1) \times L^{2}(0,1)$, which associates $\left(w_{0}, w_{1}\right)$ and $\left(y_{0}, y_{1}\right)$ such that

$$
\Lambda\left(\hat{w}_{0}, \hat{w}_{1}\right)=\left(y_{1},-y_{0}\right) .
$$




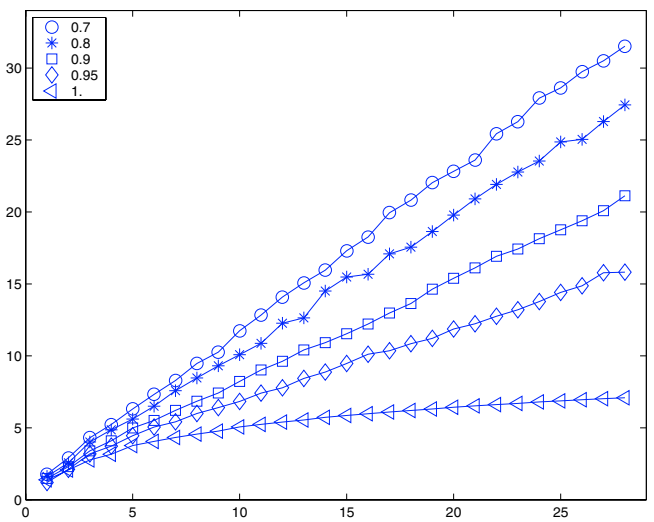

Figure 6. $\log \left(\operatorname{cond}\left(\boldsymbol{\Lambda}_{\boldsymbol{h}, \boldsymbol{\Delta} \boldsymbol{\Delta}}^{\boldsymbol{\theta}}\right)\right)$ vs. $1 / h$ for various values of $\nu=\Delta t / h \leq 1$ and $\theta=\alpha=0$. $T=2.4\left(>2 \max \left(1, \nu^{2}\right)\right)$.

If $T$ is sufficiently large, $\Lambda$ is an isomorphism from $H_{0}^{1}(0,1) \times L^{2}(0,1)$ onto $H^{-1}(0,1) \times L^{2}(0,1)[16]$, and satisfies

$$
\left\langle\Lambda\left(\hat{w}_{0}, \hat{w}_{1}\right),\left(\hat{w}_{0}, \hat{w}_{1}\right)\right\rangle=\int_{0}^{1}\left(\hat{w}_{x}(1, t)\right)^{2} \mathrm{~d} t .
$$

In order to solve numerically this linear problem, the direct approach consists of computing explicitly the matrix $\boldsymbol{\Lambda}_{\boldsymbol{h}, \boldsymbol{\theta} \boldsymbol{t}}^{\boldsymbol{\theta} \boldsymbol{\alpha}} \in \mathcal{M}_{2 J \times 2 J}(\mathbb{R})$ associated to the operator $\Lambda$ and writing that

$$
\left(\hat{\boldsymbol{w}}_{0 h}, \hat{\boldsymbol{w}}_{1 h}\right)=\left(\boldsymbol{\Lambda}_{h, \Delta t}^{\theta, \alpha}\right)^{-1}\left(\boldsymbol{y}_{1 h},-\boldsymbol{y}_{0 h}\right)
$$

In Section 7.2, we present the explicit calculation of the matrix $\Lambda_{h, \Delta t}^{\theta, \alpha}$. The indirect method consists, following [9], of using a conjugate gradient algorithm to solve (157). This approach avoids the computations of the inverse of $\boldsymbol{\Lambda}_{\boldsymbol{h}, \boldsymbol{\Delta} \boldsymbol{t}}^{\boldsymbol{\theta} \boldsymbol{\alpha}}$ and is less CPU time consuming.

\subsection{Numerical study of the condition number of $\Lambda_{h, \Delta t}^{\theta, \alpha}$}

In this section, we use the explicit expression of the positive definite matrix $\Lambda_{h, \Delta t}^{\theta, \alpha}$ to compute its condition number cond $\left(\boldsymbol{\Lambda}_{h, \Delta t}^{\boldsymbol{\theta}, \boldsymbol{\alpha}}\right)$ defined as the ratio of the largest eigenvalue over the lowest eigenvalue.

Figure 6 depicts the evolution of $\log \left(\operatorname{cond}\left(\boldsymbol{\Lambda}_{\boldsymbol{h}, \boldsymbol{\Delta} \boldsymbol{\Delta}}^{\boldsymbol{\theta}, \boldsymbol{\alpha}}\right)\right)$ as a function of $1 / h$ for $\theta=\alpha=0$ and different values of $\nu=\Delta t / h \leq 1$, i.e., for $(\theta, \alpha, \nu) \in \mathcal{S}$ corresponding to the usual centered finite difference scheme. For $\nu<1$ i.e. $(\theta, \alpha, \nu) \notin \mathcal{C}$ it appears that $\operatorname{cond}\left(\boldsymbol{\Lambda}_{\boldsymbol{h}, \boldsymbol{\theta} \boldsymbol{t}}^{\boldsymbol{\theta}, \boldsymbol{\alpha}}\right)$ increases exponentially with $1 / h$ :

$$
\operatorname{cond}\left(\Lambda_{h, \Delta t}^{\boldsymbol{\theta}, \boldsymbol{\alpha}}\right) \approx C_{\nu} \mathrm{e}^{\beta_{\nu} / h} \quad \text { for } \quad \nu<1
$$

where the coefficient $C_{\nu}>0$ and $\beta_{\nu}>0$ depend on the ratio $\nu$. On the other hand, for $\nu=1$ and $\theta=\alpha \geq 0$, i.e., such that $(\theta, \alpha, \nu) \in \boldsymbol{C}$, the behavior is only polynomial with respect to $1 / h$ (see Fig. 7 ). We obtain the fit

$$
\operatorname{cond}\left(\boldsymbol{\Lambda}_{\boldsymbol{h}, \boldsymbol{\Delta} \boldsymbol{t}}^{\boldsymbol{\theta}, \boldsymbol{\alpha}}\right) \approx \mathrm{e}^{-0.1716} h^{-2.15} \text { for } \quad \nu=1
$$

which is the slope frequently observed in well-posed finite element and finite difference schemes. Figure 7 also represents the $\log \left(\operatorname{cond}\left(\boldsymbol{\Lambda}_{\boldsymbol{h}, \boldsymbol{\theta} \boldsymbol{\theta}}^{\boldsymbol{\theta}}\right)\right)$ as a function of $\log (1 / h)$ for other values of $(\theta, \alpha, \nu) \in \mathcal{C}$. The same evolution, and in particular the same slope, is observed. We also highlight that the condition $T>2 \max \left(1, \nu^{2}\right)$ 


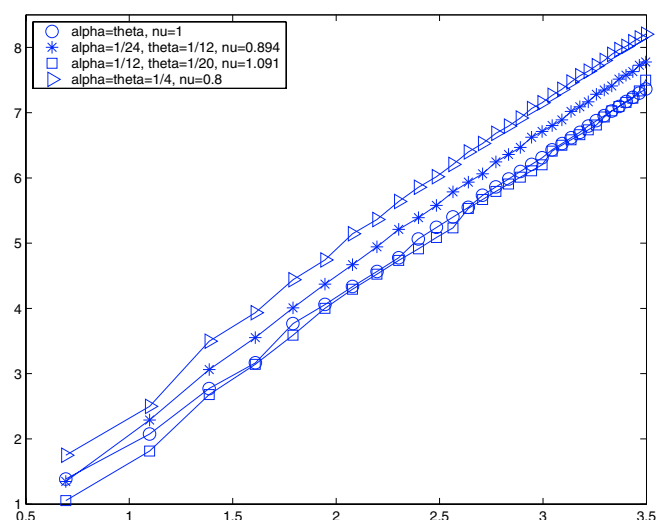

Figure $7 . \log \left(\operatorname{cond}\left(\boldsymbol{\Lambda}_{\boldsymbol{h}, \boldsymbol{\Delta} \boldsymbol{t}}^{\boldsymbol{\theta}, \boldsymbol{\alpha}}\right)\right)$ vs. $\log (1 / h) . \quad T=2.4\left(>2 \max \left(1, \nu^{2}\right)\right)$, $(\theta, \alpha, \nu) \in \mathcal{C}$

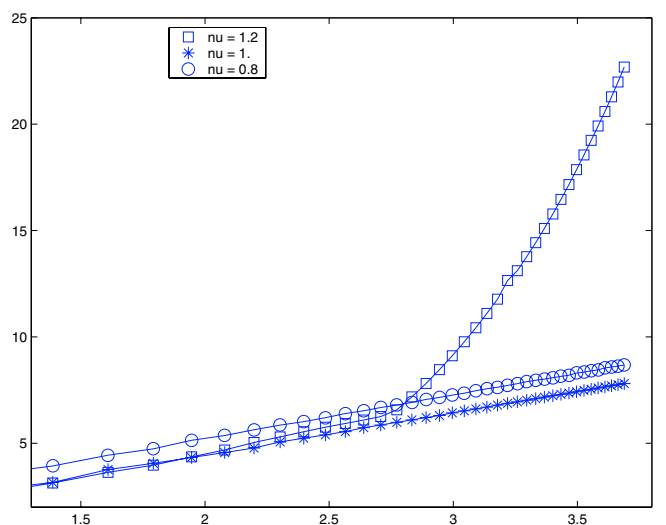

Figure 8. $\log \left(\operatorname{cond}\left(\boldsymbol{\Lambda}_{\boldsymbol{h}, \boldsymbol{\Delta} \boldsymbol{\Delta} t}^{\boldsymbol{\theta}}\right)\right)$ vs. $\log (1 / h) . \quad T=2.4, \theta=\alpha=1 / 4$ and $\nu \in\{0.8,1,1.2\}$.

is a necessary condition for the uniform controllability. For instance, let us consider $\theta=\alpha=1 / 4 \mathrm{implying}$ $(\theta, \alpha, \nu) \in \mathcal{C}$ for all $\nu>0$. Figure 8 depicts the evolution of $\log \left(\operatorname{cond}\left(\boldsymbol{\Lambda}_{\boldsymbol{h}, \boldsymbol{\Delta} \boldsymbol{t}}^{\boldsymbol{\theta}, \boldsymbol{\alpha}}\right)\right)$ in function of $\log (1 / h)$ for three values of $\nu: 0.8,1$ and 1.2. For $\nu=0.8$ and $\nu=1$, we obtain a polynomial evolution. On the contrary, for $\nu=1.2$, which implies $2 \max \left(1, \nu^{2}\right)=2 \times(1.2)^{2}>T=2.4$, the evolution blows up for a small enough value of $h$. The evolution of the condition number of $\Lambda_{h, \Delta t}^{\theta, \alpha}$ associated to the HUM operator $\Lambda$ indicates clearly that the numerical resolution of the control problem with the usual finite difference scheme $\left(\mathcal{S}_{h, \Delta t}^{0,0}\right), h \neq \Delta t$ is not well-posed. As we will see in the next paragraph, the exponential behavior of $\operatorname{cond}\left(\boldsymbol{\Lambda}_{\boldsymbol{h}, \boldsymbol{\theta} \boldsymbol{t}}^{\boldsymbol{\theta}}\right)$ may produce, for a small enough value of $h$, a bad approximation of $\left(\Lambda_{\boldsymbol{h}, \boldsymbol{\Delta} t}^{\theta, \boldsymbol{\alpha}}\right)^{-1}$ used with a direct method, and a divergence of an iterative approach, like the conjugate gradient algorithm. On the contrary, the use of the scheme $\left(\mathcal{S}_{h, \Delta t}^{\theta, \alpha}\right)$ with $(\theta, \alpha, \nu) \in \mathcal{C}$ and $T>2 \max \left(1, \nu^{2}\right)$ permits to recover the usual polynomial behavior of the condition number. We remark that these different behaviors are independent of the regularity of the initial condition.

\subsection{Numerical examples}

In the rest of this section, we present some numerical experiments. For brevity, we consider only one initial condition. We take the most singular situation where the initial condition $y_{0}$ is discontinuous. We compare the result obtained from the usual finite difference scheme $(\mathrm{FDS})$, i.e. $\left(\mathcal{S}_{h, 0}^{0,0}\right), \Delta t<h$, the scheme $\left(\mathcal{S}_{h, \Delta}^{\theta, \alpha}\right)$ for $(\theta, \alpha, \nu) \in \mathcal{C}(\mathrm{MDS})$ and the bi-grid method (BI-GRID) introduced in [9] (see also [1]). When the initial conditions $\left(y_{0}, y_{1}\right)$ are both continuous, the usual centered scheme (FDS) provides good numerical results, and convergence of the discrete control is observed (see for instance [5] for numerical simulations in 2-D). This point is in agreement with the theoretical result at the semi-discrete level presented in [17]. The loss of uniform controllability occurs when the initial velocity $y_{1}$ is discontinuous. The well-known Glowinski-Li-Lions test enters in this case (see [9]).

On the interval $[0,1]$, we consider the following discontinuous initial conditions associated to the wave equation (1):

$$
y_{0}(x)=\left\{\begin{array}{cc}
16 x & x \in[0,1 / 2] \\
0 & x \in] 1 / 2,1]
\end{array} \quad ; \quad y_{1}(x)=0\right.
$$



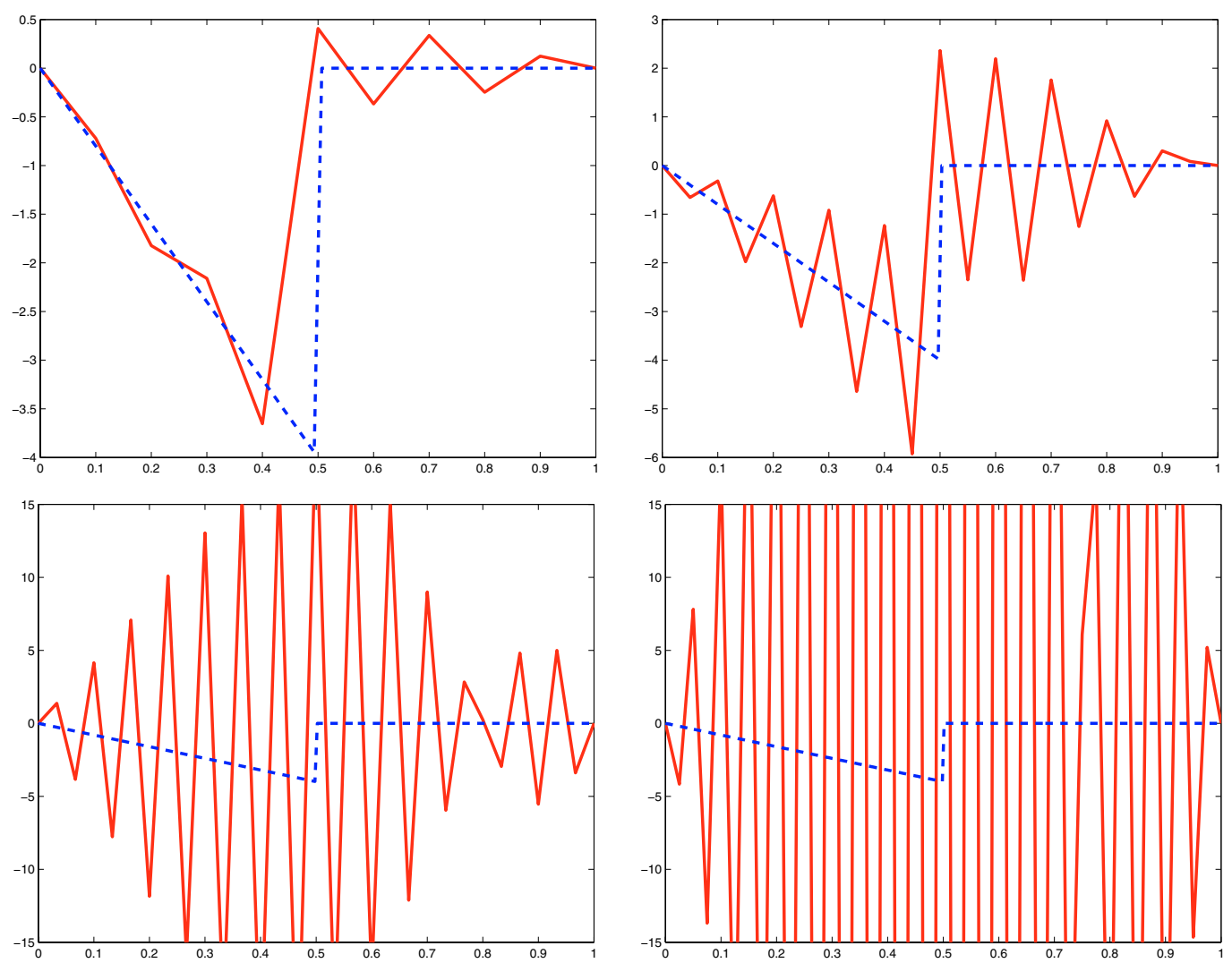

Figure 9. (FDS) $P\left(\hat{\boldsymbol{w}}_{1 \boldsymbol{h}}\right)(x)$ vs. $x \in[0,1], \nu=0.98, T=2.4$ and $h=1 / 10$ (top left), $h=1 / 20$ (top right), $h=1 / 30$ (bottom left), $h=1 / 40$ (bottom right).

Let us take $T=2.4$. The control $v$ associated to (1) which minimizes the energy $J$ with minimal $L^{2}$-norm is the following discontinuous function:

$$
v(t)=\left\{\begin{array}{cc}
0 & t \in[0,0.9] \cup[1.9, T] \\
8(t-1.4) & t \in] 0.9,1.9[
\end{array},\right.
$$

leading to $\|v\|_{L^{2}(0, T)}=4 / \sqrt{3} \approx 2.3094$. The corresponding initial conditions of the forward problem (6) are

$$
\hat{w}_{0}(x)=0 \quad ; \quad \hat{w}_{1}(x)=\left\{\begin{array}{cl}
-8 x & x \in[0,1 / 2[ \\
0 & x \in[1 / 2,1]
\end{array} .\right.
$$

\subsubsection{Usual centered finite scheme $\left(\mathcal{S}_{h, \Delta t}^{0,0}\right), \Delta t \leq h(F D S)$}

Let us use the usual centered scheme $\left(\mathcal{S}_{h, \Delta t}^{0,0}\right)$, with $\nu=0.98$ coupled with the direct inverse method (159). Figures 9 represent $\left(\hat{\boldsymbol{w}}_{1 \boldsymbol{h}}\right)$, for $h=1 / 10,1 / 20,1 / 30$ and $1 / 40$, obtained by (159). On these figures, the curve in dash dot represents the solution $\hat{w}_{1}$ of the continuous system defined in (164). As expected, due to the irregularity of the initial condition $y_{0}$ which exhibits high frequency components of the solution $y$, the method produces a divergent sequence. Figure 10 represents the discrete control $\boldsymbol{v}_{\boldsymbol{h}}$ (and more precisely $P\left(\boldsymbol{v}_{\boldsymbol{h}}\right)$ ) associated to $\left(\hat{\boldsymbol{w}}_{\mathbf{0} \boldsymbol{h}}, \hat{\boldsymbol{w}}_{\mathbf{1} \boldsymbol{h}}\right)$. The curve in dash dot represents the control $v$ defined in (163). For $\nu<1$, it appears that the $L^{2}$-norm of the control diverges when $h$ tends to zero with a rate higher than $\exp (1 / h)(\operatorname{see}$ Fig. 11$)$. For $\nu=1$, $\left\|Q\left(\boldsymbol{v}_{\boldsymbol{h}}\right)\right\|_{L^{2}(0, T)}$ converges to a finite value when $h=\Delta t$ goes to zero. 

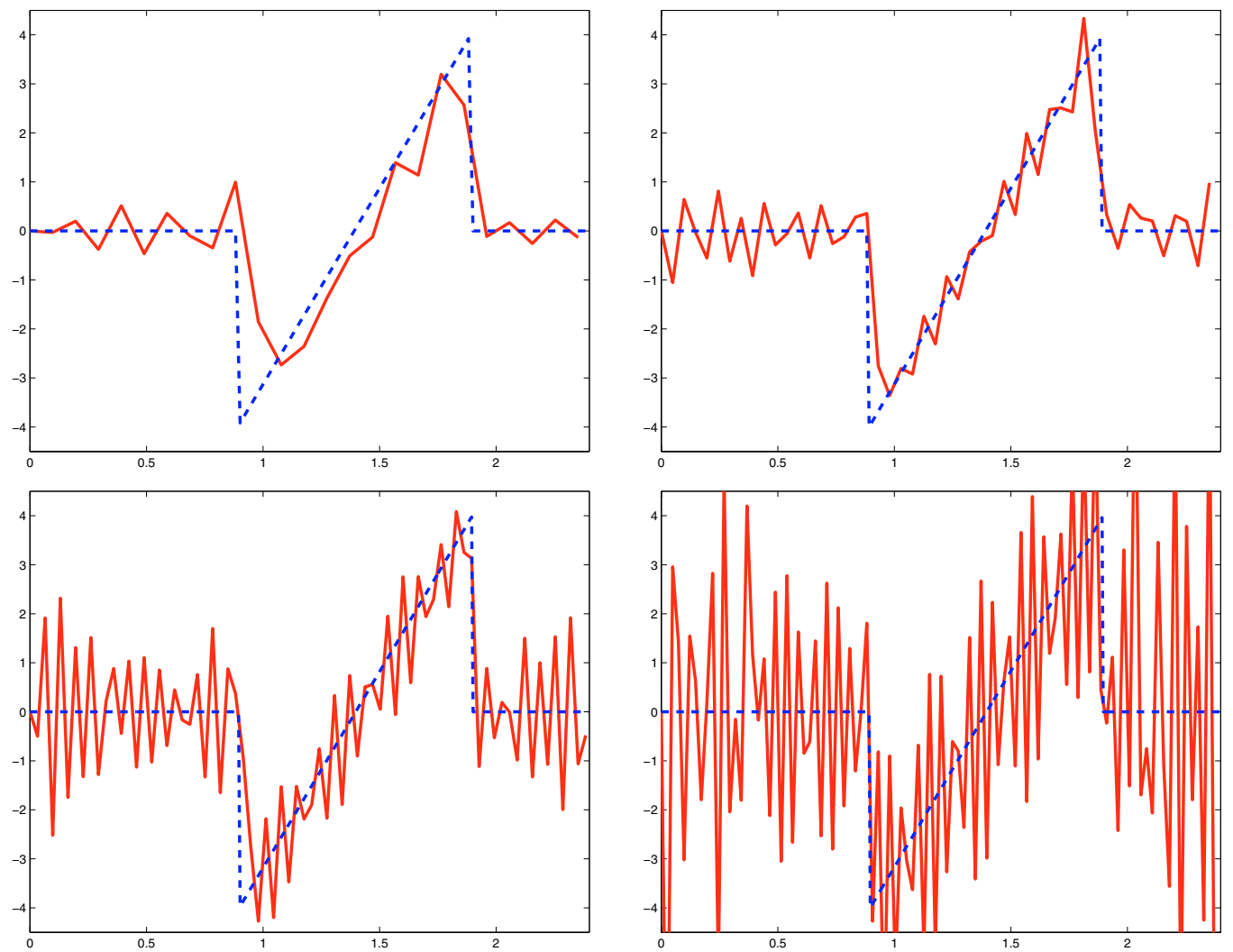

Figure 10. (FDS): Control $P\left(\boldsymbol{v}_{\boldsymbol{h}}\right)(t)$ vs. $t \in[0, T], \nu=0.98, T=2.4$ and $h=1 / 10$ (top left), $h=1 / 20$ (top right), $h=1 / 30$ (bottom left), $h=1 / 40$ (bottom right).

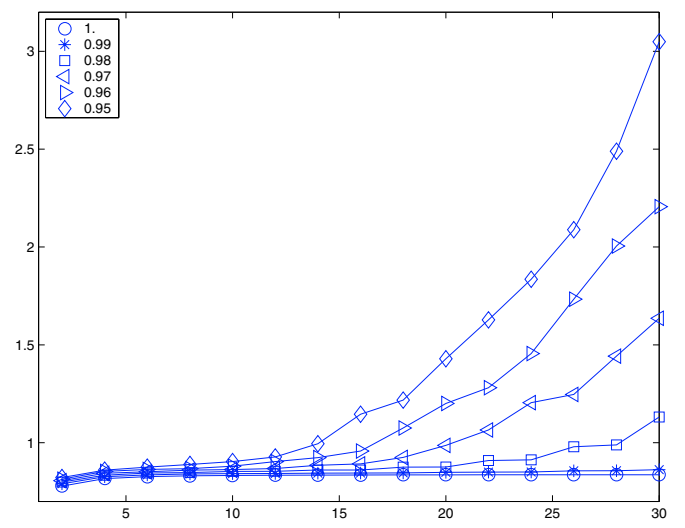

Figure 11. (FDS) $\log \left(\left\|Q\left(\boldsymbol{v}_{\boldsymbol{h}}\right)\right\|_{L^{2}(0, T)}\right)$ vs. $1 / h$ for different values of $\nu . T=2.4$.

The use of the indirect inverse method - conjugate gradient algorithm - does not produce a better result. The error produced by this algorithm at the $n$-th iteration is of order (see [11])

$$
\left(\frac{\sqrt{\operatorname{cond}\left(\boldsymbol{\Lambda}_{\boldsymbol{h}, \boldsymbol{\Delta} t}^{\theta, \boldsymbol{\alpha}}\right)}-1}{\sqrt{\operatorname{cond}\left(\boldsymbol{\Lambda}_{\boldsymbol{h}, \boldsymbol{\theta} \boldsymbol{\alpha}}^{\theta, \boldsymbol{}}\right)}+1}\right)^{n}
$$



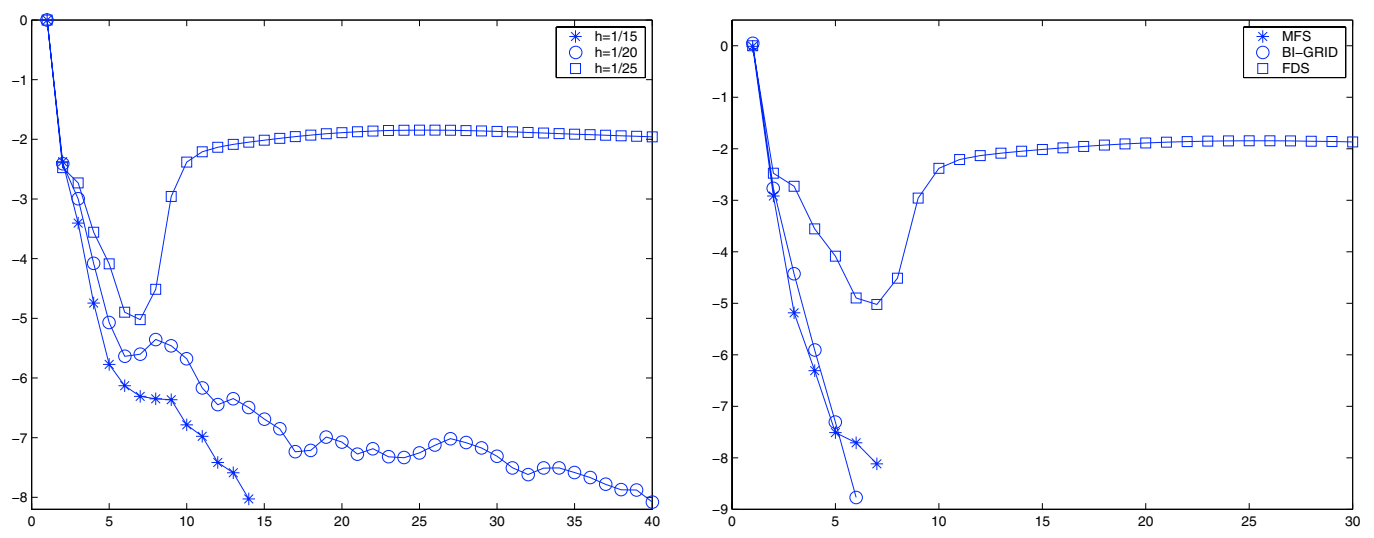

FIGURE 12. Log10(Relative error of the residual) vs. iteration of the conjugate gradient algorithm - Left: (FDS) with $\nu=0.99$ and $h=1 / 15,1 / 20,1 / 25$ - Right: $h=1 / 25$ (FDS) with $\nu=0.99,(\mathrm{MFS})$ and Bi-Grid with $\nu=0.8$.

Hence, if the condition number is of order $h^{-2}$, the error at the $n-t h$ iteration is of order $(1-2 h)^{n} \approx 1-2 h n$, whereas if the condition number is of order $\exp (1 / h)$, the error is of order $(1-2 \exp (-1 /(2 h)))^{n} \approx 1-$ $2 n \exp (-1 /(2 h))$. In the last situation, the convergence of the algorithm is not guaranteed. Figure 12-left depicts the relative error of the residual associated to the iterative algorithm for three values of $h$. For the smallest one, i.e. $h=1 / 25$, the algorithm does not converge. On the contrary, the use of the scheme $\left(S_{h, \Delta t}^{\theta, \alpha}\right)$ (MFS) with $(\theta, \alpha, \nu) \in \boldsymbol{C}$ produces a decreasing relative error and a fast convergence (see Fig. 12-right). Let us note however that the behavior of the error for $h=1 / 25$ leads to an interesting remark. After a few iterations, the error reaches the relative value $\approx 10^{-2}$ and then increases. This means that the usual centered scheme is able to solve the approximate controllability problem where the goal is to drive the solution to an $\epsilon$-state such that

$$
\|y(., T)\|_{L^{2}(0,1)}+\left\|y^{\prime}(., T)\right\|_{H^{-1}(0,1)} \leq \epsilon, \quad \epsilon>0 .
$$

Actually, in this case, the approximate control of the full discrete system can be obtained by minimizing the functional

$$
\mathcal{J}_{h}^{\epsilon}\left(\boldsymbol{w}_{\mathbf{0} \boldsymbol{h}}, \boldsymbol{w}_{\mathbf{1} \boldsymbol{h}}\right)=\mathcal{J}_{h}\left(\boldsymbol{w}_{\mathbf{0} \boldsymbol{h}}, \boldsymbol{w}_{\mathbf{1} \boldsymbol{h}}\right)+\epsilon\left\|\boldsymbol{w}_{\mathbf{0} \boldsymbol{h}}, \boldsymbol{w}_{\mathbf{1} \boldsymbol{h}}\right\|_{1}^{2}
$$

leading to an observability constant $C(h, T)+\epsilon$ uniformly bounded from below with respect to $h$ and $\Delta t$.

\subsubsection{Modified finite difference scheme $\left(\mathcal{S}_{h, \Delta t}^{\theta, \alpha}\right),(\theta, \alpha, \nu) \in \mathcal{C}(M F S)$}

Let us now present some results associated to the scheme $\left(\mathcal{S}_{h, \Delta t}^{\theta, \alpha}\right)$ and $(\theta, \alpha, \nu) \in \boldsymbol{C}$. The minimization problem is solved using a conjugate gradient algorithm. Convergence is assumed to be attained when the relative error on the residual is less than $\varepsilon=1 . E-08$. Table 1 summarizes the results obtained with $(\theta, \alpha, \nu) \approx$ $(1 / 20,1 / 12,1.095445)$. The results illustrate the convergence of the discrete control. Convergence is obtained after a number of iterations which is independent of $h$. The initial condition being irregular, Theorem 2.8 only ensures weak convergence in the $L^{2}$-norm. We numerically observe the strong convergence of the discrete control $\left(\boldsymbol{v}_{\boldsymbol{h}}\right)_{h}$ (and also of $\hat{\boldsymbol{w}}_{\mathbf{1} \boldsymbol{h}}$ ) with low rate (see Fig. 13). Figures 14 and 15 then depict the discrete control $P\left(\boldsymbol{v}_{\boldsymbol{h}}\right)$ and initial forward condition $P\left(\hat{\boldsymbol{w}}_{1 \boldsymbol{h}}\right)$ for $h=1 / 21, h=1 / 41, h=1 / 81$ and $h=1 / 161$. The pictures highlight the well known numerical Gibbs phenomenon at the discontinuity of the control, i.e., at $t=0.9$ and $t=1.9$. It is also interesting to note that the system is stabilized at time $t=1.9$ lower than $T=2.4$.

In the same spirit, Table 2 and Figures 16 display the results obtained with $(\theta, \alpha, \nu) \approx(1 / 12,1 / 24,0.894427)$. For these value leading to $\nu<1$, the results are slightly deteriorated. Observe for instance the ratio $\left\|\boldsymbol{y}_{\boldsymbol{h}}(T)\right\|_{L^{2}(0,1)} /\left\|\boldsymbol{y}_{\boldsymbol{h}}(0)\right\|_{L^{2}(0,1)}$ which indicates the decreasing rate of the solution for the $L^{2}$-norm. This ratio is greater than the ratio obtained in Table 1 . We also note that the algorithm requires more iterations in 
TABLE 1. (MFS) Results obtained with MFS for $(\theta, \alpha, \nu) \approx(1 / 20,1 / 12,1.095445)$.

\begin{tabular}{|c|c|c|c|c|c|}
\hline & $h=1 / 21$ & $h=1 / 41$ & $h=1 / 81$ & $h=1 / 161$ & $h=1 / 321$ \\
\hline Nb. iteration & 9 & 5 & 4 & 4 & 4 \\
\hline$\left\|\hat{\boldsymbol{w}}_{\mathbf{O}}\right\|_{L^{2}(0,1)}$ & 0.000846908 & 0.000149009 & $2.63 \mathrm{e}-006$ & 7.04913e-009 & $4.35578 \mathrm{e}-013$ \\
\hline$\left\|\hat{\boldsymbol{w}}_{\mathbf{O}}\right\|_{H^{1}(0,1)}$ & 0.00780284 & 0.00175735 & $4.03116 \mathrm{e}-005$ & $1.49863 \mathrm{e}-007$ & $9.9983 \mathrm{e}-012$ \\
\hline$\frac{\left\|\hat{\boldsymbol{w}}_{1}-\hat{\boldsymbol{w}}_{1 \boldsymbol{h}}\right\|_{L^{2}(0,1)}}{\left\|\hat{\boldsymbol{w}}_{1}\right\|_{L^{2}(0,1)}}$ & 0.0619121 & 0.0442768 & 0.0314859 & 0.02233 & 0.0158137 \\
\hline$\frac{\left\|\boldsymbol{y}_{\mathbf{0}}-\boldsymbol{y}_{\mathbf{0} \boldsymbol{h}}\right\|_{L^{2}(0, T)}}{\left\|\boldsymbol{y}_{\mathbf{0}}\right\|_{L^{2}(0,1)}}$ & 0.000171413 & $3.95289 \mathrm{e}-005$ & $4.07445 \mathrm{e}-005$ & $2.69717 \mathrm{e}-005$ & $1.92028 \mathrm{e}-005$ \\
\hline$\left\|\boldsymbol{y}_{\mathbf{1} \boldsymbol{h}}\right\|_{H^{-1}(0,1)}$ & 0.00053694 & 0.000184141 & $3.98393 \mathrm{e}-005$ & $1.61162 \mathrm{e}-007$ & $1.14645 \mathrm{e}-011$ \\
\hline$\frac{\left\|\boldsymbol{v}_{\boldsymbol{h}}-\boldsymbol{v}\right\|_{L^{2}(0, T)}}{\|\boldsymbol{v}\|_{L^{2}(0, T)}}$ & 0.324091 & 0.272798 & 0.232061 & 0.207709 & 0.16381 \\
\hline$\left\|\boldsymbol{v}_{\boldsymbol{h}}\right\|_{L^{2}(0, T)}$ & 2.13703 & 2.21929 & 2.26328 & 2.28606 & 2.29766 \\
\hline$\frac{\left\|\boldsymbol{y}_{\boldsymbol{h}}(T)\right\|_{L^{2}(0,1)}}{\left\|\boldsymbol{y}_{\boldsymbol{h}}(0)\right\|_{L^{2}(0,1)}}$ & 0.00580092 & 0.00141641 & $5.37207 \mathrm{e}-005$ & $2.07521 \mathrm{e}-005$ & $1.44698 \mathrm{e}-005$ \\
\hline
\end{tabular}

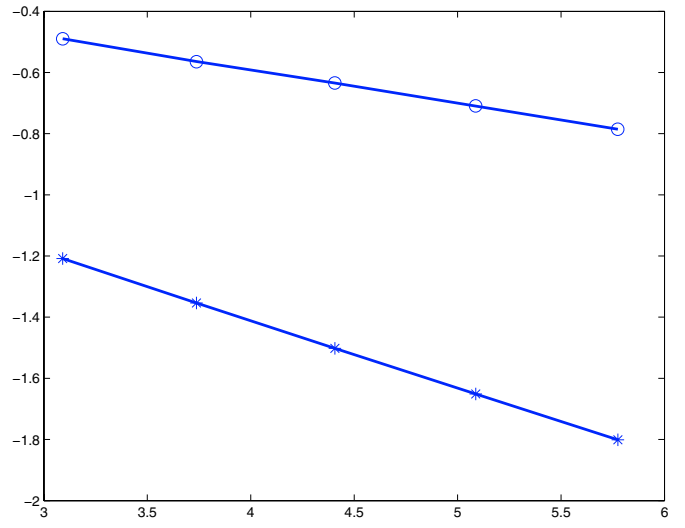

FIGURE 13. Relative error $\frac{\left\|\boldsymbol{v}_{h}-\boldsymbol{v}\right\|_{0, \partial \Omega}}{\|\boldsymbol{v}\|_{0, \partial \Omega}} \approx \mathrm{e}^{-0.3475} h^{0.2530}$ ( ○) and $\frac{\left\|\hat{\boldsymbol{w}}_{1 h}-\hat{\boldsymbol{w}}_{1}\right\|_{0, \partial \Omega}}{\left\|\hat{\boldsymbol{w}}_{1}\right\|_{0, \partial \Omega}}=\mathrm{e}^{-1.2149} h^{0.5082}$

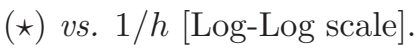

order to converge. If we exclude the particular case $\nu=1$ leading to exact values, we observe that the best results in terms of convergence rate and relative error are obtained for the largest value of $\nu$ that ensures uniform controllability, i.e. $\nu \approx \sqrt{T / 2}$. This was the case with $\theta=1 / 20$ and $\alpha=1 / 12$.

\subsubsection{Bi-grid finite difference scheme $\nu \leq 1$ (Bi-Grid)}

To conclude this section, we now compare our scheme with the scheme proposed in [9] based on a bi-grid strategy. It consists of performing some parts of the algorithm on a coarse grid. This projection has the effect to regularize the initial condition. [9] presents a numerical study that indicates the efficiency of the method. In our example, the results are given by Table 3, Figures 17 and 18. At a first glance, the result seems very good. In particular, the number of iterations to reach convergence is low (see also Fig. 12, right). However, the ratio $\left\|\boldsymbol{y}_{\boldsymbol{h}}(T)\right\|_{L^{2}(0,1)} /\left\|\boldsymbol{y}_{\boldsymbol{h}}(0)\right\|_{L^{2}(0,1)}$ in Table 3 indicates that the control obtained does not drive the solution at rest at time $T$. This result is not a contradiction. The theoretical proof of convergence for this method remains to be done. It was recently proved in [22] that the control obtained by this method only controls the projection of the discrete wave system on the coarse mesh. Figure 18 shows that the sequence $\left(P\left(\hat{\boldsymbol{w}}_{1 \boldsymbol{h}}\right)\right)_{h}$ converges to a function, say $\tilde{w}_{1}$, slightly different from $\hat{w}_{1}$ at the discontinuity $x=1 / 2$. According to [22], we 

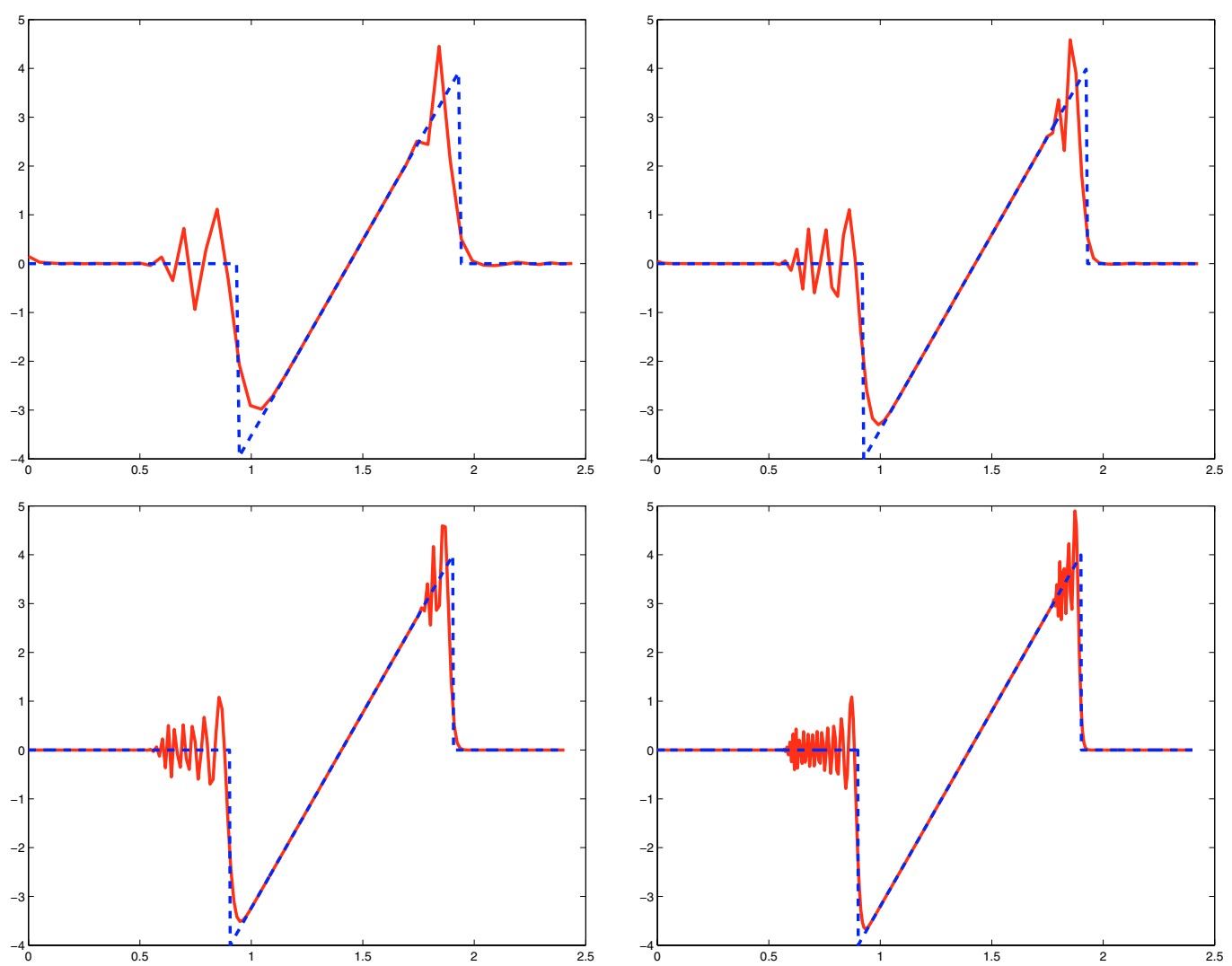

Figure 14. (MFS) Control $P\left(\boldsymbol{v}_{\boldsymbol{h}}\right)(t)$ vs. $t \in[0, T]-(\theta, \alpha, \nu) \approx(1 / 20,1 / 12,1.095445), T=2.4$ and $h=1 / 21,1 / 41,1 / 81,1 / 161$.

TABLE 2. (MFS) Results obtained with MFS for $(\theta, \alpha, \nu) \approx(1 / 12,1 / 24,0.894427)$.

\begin{tabular}{|c|c|c|c|c|c|}
\hline & $h=1 / 21$ & $h=1 / 41$ & $h=1 / 81$ & $h=1 / 161$ & $h=1 / 321$ \\
\hline Nb. iteration & 11 & 11 & 11 & 11 & 11 \\
\hline$\left\|\hat{\boldsymbol{w}}_{\mathbf{O h}}\right\|_{L^{2}(0,1)}$ & 0.0080438 & 0.00279635 & 0.00133671 & 0.000474703 & 0.000171095 \\
\hline$\left\|\hat{\boldsymbol{w}}_{\mathbf{0 h}}\right\|_{H^{1}(0,1)}$ & 0.121 & 0.0882796 & 0.067398 & 0.046819 & 0.0336129 \\
\hline$\frac{\left\|\hat{\boldsymbol{w}}_{\boldsymbol{1}}-\hat{\boldsymbol{w}}_{\boldsymbol{1} \boldsymbol{h}}\right\|_{L^{2}(0,1)}}{\left\|\hat{\boldsymbol{w}}_{\mathbf{1}}\right\|_{L^{2}(0,1)}}$ & 0.0348545 & 0.0251213 & 0.0200815 & 0.0136948 & 0.00985628 \\
\hline$\frac{\left\|\boldsymbol{y}_{\mathbf{0}}-\boldsymbol{y}_{\mathbf{O}} \boldsymbol{h}\right\|_{L^{2}(0,1)}}{\left\|\boldsymbol{y}_{\mathbf{0}}\right\|_{L^{2}(0,1)}}$ & 0.000879055 & 0.000627187 & 0.000506205 & 0.000370724 & 0.000263518 \\
\hline$\left\|\boldsymbol{y}_{1 \boldsymbol{h}}\right\|_{H^{-1}(0,1)}$ & 0.00235356 & 0.00189625 & 0.00139522 & 0.00102185 & 0.000699627 \\
\hline$\frac{\left\|\boldsymbol{v}_{\boldsymbol{h}}-\boldsymbol{v}\right\|_{L^{2}(0, T)}}{\|\boldsymbol{v}\|_{L^{2}(0, T)}}$ & 0.363298 & 0.311467 & 0.24415 & 0.185029 & 0.160107 \\
\hline$\left\|\boldsymbol{v}_{\boldsymbol{h}}\right\|_{L^{2}(0, T)}$ & 2.14065 & 2.22097 & 2.26356 & 2.28646 & 2.29783 \\
\hline$\frac{\left\|\boldsymbol{y}_{\boldsymbol{h}}(T)\right\|_{L^{2}(0,1)}}{\left\|\boldsymbol{y}_{\boldsymbol{h}}(0)\right\|_{L^{2}(0,1)}}$ & 0.008666 & 0.016713 & 0.00667307 & 0.00168309 & 0.0015795 \\
\hline
\end{tabular}



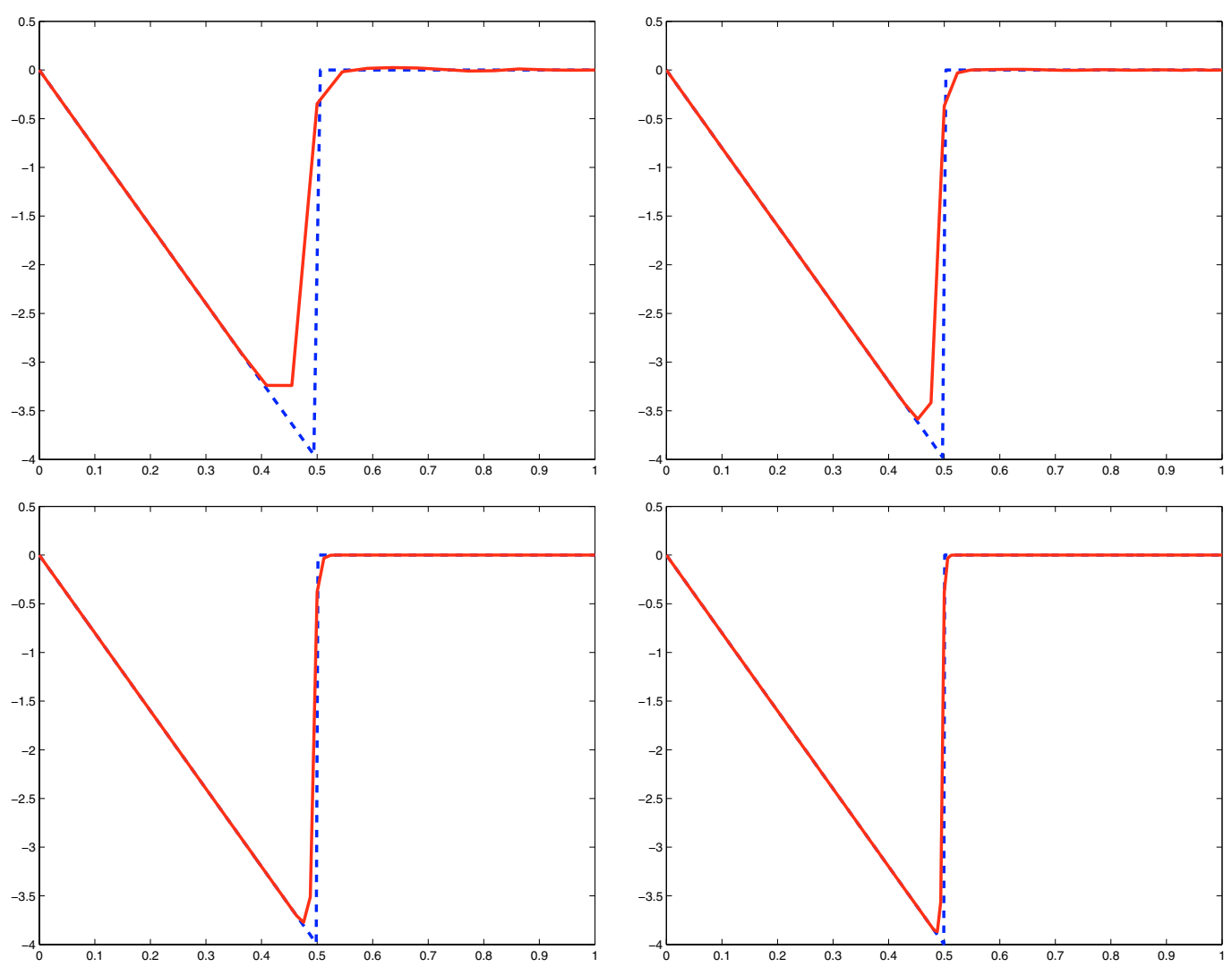

Figure 15. (MFS) $P\left(\hat{\boldsymbol{w}}_{1 \boldsymbol{h}}\right)(x)$ vs. $x \in[0,1]-(\theta, \alpha, \nu) \approx(1 / 20,1 / 12,1.095445), T=2.4$ and $h=1 / 21,1 / 41,1 / 81,1 / 161$.

may conjecture that the initial conditions $\left(\tilde{y}_{0}, \tilde{y}_{1}\right)$, associated to $\left(\tilde{w}_{0}, \tilde{w}_{1}\right)$ using $(157)$, are a kind of regularization of $\left(y_{0}, y_{1}\right)$. In our example, the bi-grid procedure has no regularization effect on $y_{0}$. The projection of $y_{0}$ on a coarse grid remains discontinuous with a jump independent of $h$. This example shows that the test on the relative residual commonly used in the literature should be replaced or at least confirmed by a test on the quantity $\left\|\boldsymbol{y}_{\boldsymbol{h}}(T)\right\|_{L^{2}(0,1)} /\left\|\boldsymbol{y}_{\boldsymbol{h}}(0)\right\|_{L^{2}(0,1)}$ or $E_{h}(T) / E_{h}(0)$, where $E_{h}$ designates the discrete energy associated to the system (21). We add that these numerical pathologies remain if we consider $\nu=1$. On the other hand, if we consider a continuous regularization of $y_{0}$, the Bi-Grid method becomes very efficient for $\nu \leq 1$.

For regular initial data, the implicit scheme (MFS) is comparable in term of CPU time with the explicit scheme obtained by the Bi-Grid method. The implicit character (inversion of the tri-diagonal mass matrix $\left.M_{0}^{\theta_{\nu}}\right)$ is compensated by the less restrictive stability and controllability condition $\Delta t \leq h \sqrt{T / 2}$. For the BiGrid method, we recall that this condition is $\Delta t \leq h$. In this respect, it may be interesting to couple, for regular initial conditions, these two methods. This will produce an implicit scheme with part of the computations performed on the coarse grid.

\section{Conclusions}

In the framework of the exact boundary controllability, we have introduced in this work a parametrized implicit and consistent scheme for the 1-D wave equation. The use of a discrete version of an Ingham inequality has then allowed us to determine the subset of these parameters which leads to uniform observability and controllability properties with respect to the parameters of discretization. The resulting scheme, similar to 

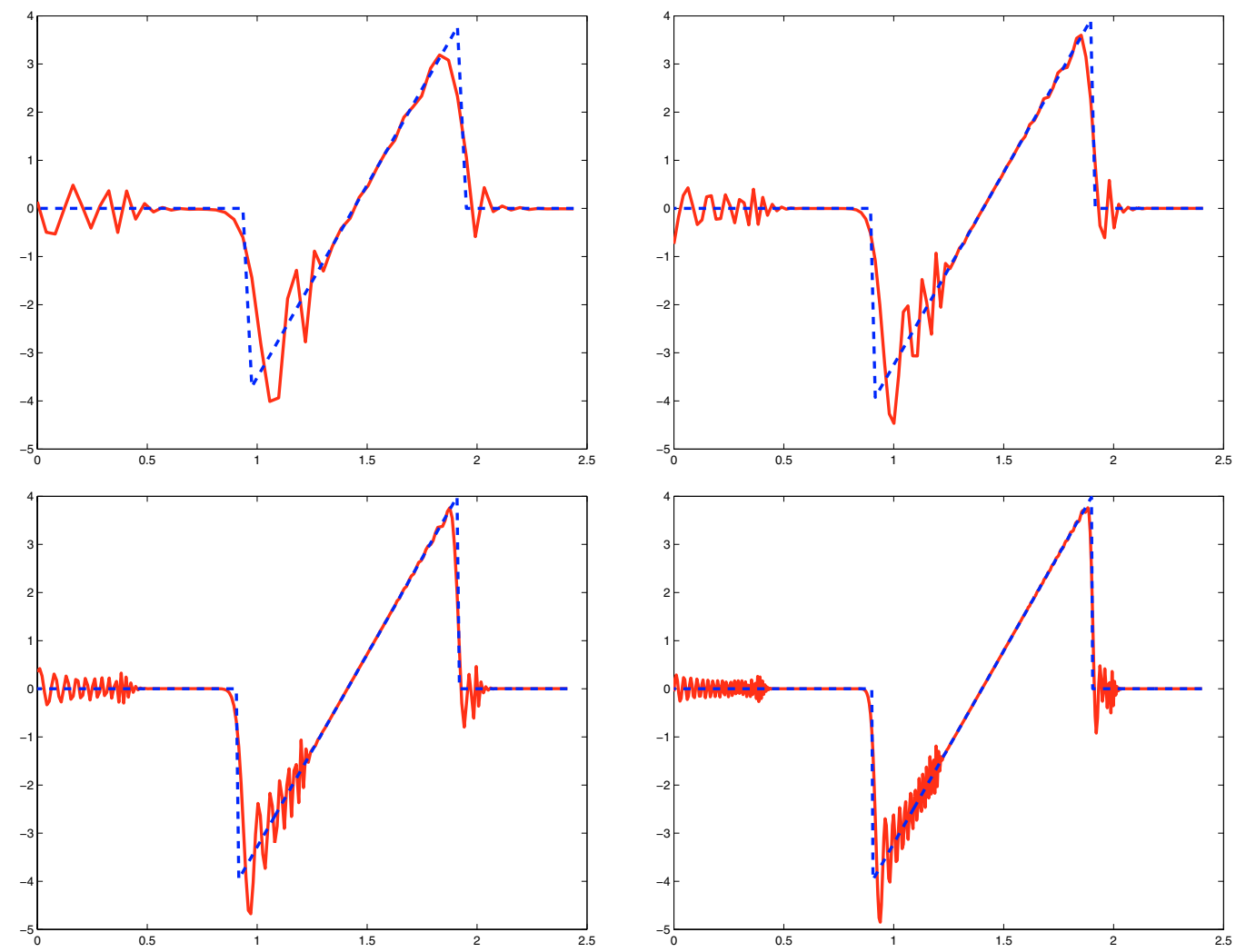

Figure 16. (MFS) Control $P\left(\boldsymbol{v}_{\boldsymbol{h}}\right)(t)$ vs. $t \in[0, T]-(\theta, \alpha, \nu) \approx(1 / 12,1 / 24,0.894427), T=2.4$ and $h=1 / 21,1 / 41,1 / 81,1 / 161$.

TABLe 3. (Bi-Grid) Results obtained with BI-GRID for different values of $h-\nu=0.5$.

\begin{tabular}{|c|c|c|c|c|c|}
\hline & $h=1 / 21$ & $h=1 / 41$ & $h=1 / 81$ & $h=1 / 161$ & $h=1 / 321$ \\
\hline Nb. iteration & 7 & 8 & 7 & 6 & 6 \\
\hline$\left\|\hat{\boldsymbol{w}}_{\mathbf{0}}\right\|_{L^{2}(0,1)}$ & 0.00569164 & 0.00171293 & 0.000773171 & 0.000245656 & $6.82782 \mathrm{e}-005$ \\
\hline$\left\|\hat{\boldsymbol{w}}_{\mathbf{O h}}\right\|_{H^{1}(0,1)}$ & 0.0424603 & 0.0249753 & 0.0184336 & 0.0132289 & 0.009157 \\
\hline$\frac{\left\|\hat{\boldsymbol{w}}_{1}-\hat{\boldsymbol{w}}_{1 h}\right\|_{L^{2}(0,1)}}{\left\|\hat{\boldsymbol{w}}_{1}\right\|_{L^{2}(0,1)}}$ & 0.105889 & 0.077454 & 0.0550586 & 0.0391519 & 0.0278292 \\
\hline$\frac{\left\|y_{\mathbf{0}}-\boldsymbol{y}_{\mathrm{O} h}\right\|_{L^{2}(0,1)}}{\left\|\boldsymbol{y}_{\mathbf{0}}\right\|_{L^{2}(0,1)}}$ & 0.267206 & 0.195063 & 0.139463 & 0.0991343 & 0.070346 \\
\hline$\left\|\boldsymbol{y}_{\mathbf{1} \boldsymbol{h}}\right\|_{H^{-1}(0,1)}$ & 0.0957837 & 0.0621117 & 0.0453737 & 0.0324067 & 0.0226495 \\
\hline$\frac{\left\|\boldsymbol{v}_{\boldsymbol{h}}-\boldsymbol{v}\right\|_{0, \partial \Omega}}{\|\boldsymbol{v}\|_{L^{2}(0, T)}}$ & 0.376652 & 0.310142 & 0.255145 & 0.208134 & 0.166963 \\
\hline$\left\|\boldsymbol{v}_{\boldsymbol{h}}\right\|_{L^{2}(0, T)}$ & 2.29027 & 2.30346 & 2.3068 & 2.30823 & 2.30888 \\
\hline$\frac{\left\|\boldsymbol{y}_{\boldsymbol{h}}(T)\right\|_{L^{2}(0,1)}}{\left\|\boldsymbol{y}_{\boldsymbol{h}}(0)\right\|_{L^{2}(0,1)}}$ & 0.186798 & 0.132 & 0.0961499 & 0.0689093 & 0.049955 \\
\hline
\end{tabular}

those obtained after a Tychonov regularization, is an implicit one where some differential terms of order four, factors of $h^{2}$ and $\Delta t^{2}$ appear. Under the stability and uniform controllability condition $\Delta t<h \sqrt{T / 2}$, we have shown, using a Fourier analysis, the strong convergence of the discrete control toward the continuous control 

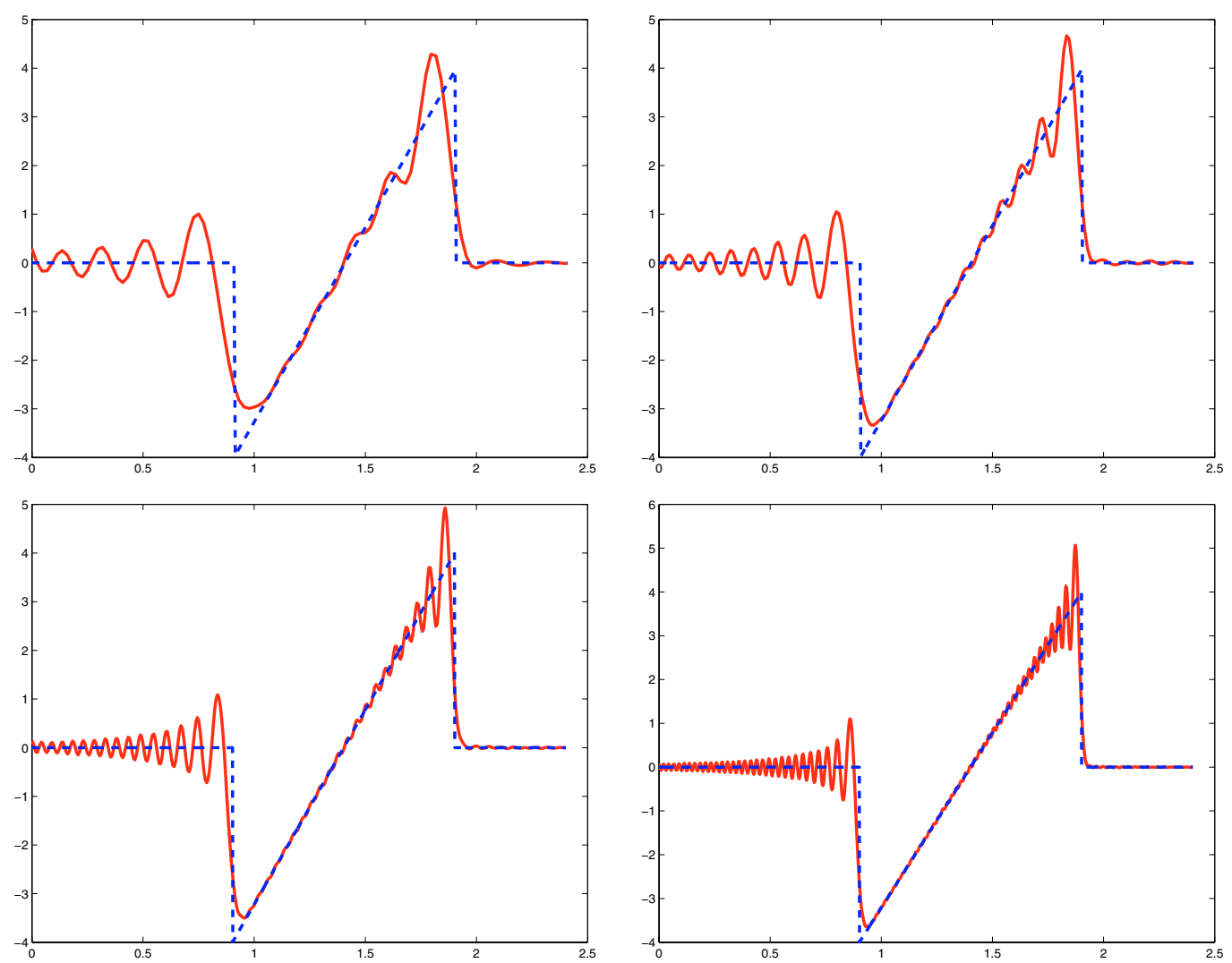

Figure 17. (Bi-Grid) Control $P\left(\boldsymbol{v}_{\boldsymbol{h}}\right)(t)$ vs. $t \in[0, T]$ obtained with the bi-grid method $\nu=0.5, T=2.4$ and $h=1 / 21,1 / 41,1 / 81,1 / 161$.

with minimal $L^{2}$-norm. The numerical experiments presented are in very good agreement with the theory. Concerning the CPU time, the scheme appears comparable with the multi-grid method. The implicit character of the scheme is compensated by a less restrictive condition on the ratio $\Delta t / h$. It will be now interesting to study whether or not it is possible to extend or adapt this kind of fully discrete analysis to more complicated models, like for instance the elasto-dynamic model or to higher dimension, for which contrary to the 1-D wave system, no exact scheme is known.

\section{Appendix}

\subsection{Appendix 1: Proof of the relation (122)}

The aim of this first appendix is to show the equality (122), i.e.,

$$
\left\|\boldsymbol{y}_{\mathbf{o h}}, \boldsymbol{y}_{\mathbf{1} \boldsymbol{h}}\right\|_{-1}^{2}=\frac{1}{2} \sum_{k=1}^{J}\left(\cos ^{2}\left(\frac{k \pi h}{2}\right)+\nu^{2} \sin ^{2}\left(\frac{k \pi h}{2}\right)\right)\left(\left(\bar{a}_{k, h}\right)^{2}+\left(\frac{\bar{b}_{k, h}}{\mu_{k, h}}\right)^{2}\right) \text {. }
$$

In order to simplify the expressions of this appendix, we note

$$
c_{k}=\cos \left(\frac{k \pi h}{2}\right), \quad s_{k}=\sin \left(\frac{k \pi h}{2}\right), \quad c_{1 k}=\cos \left(\mu_{k, h} \Delta t\right), \quad s_{1 k}=\sin \left(\mu_{k, h} \Delta t\right) .
$$



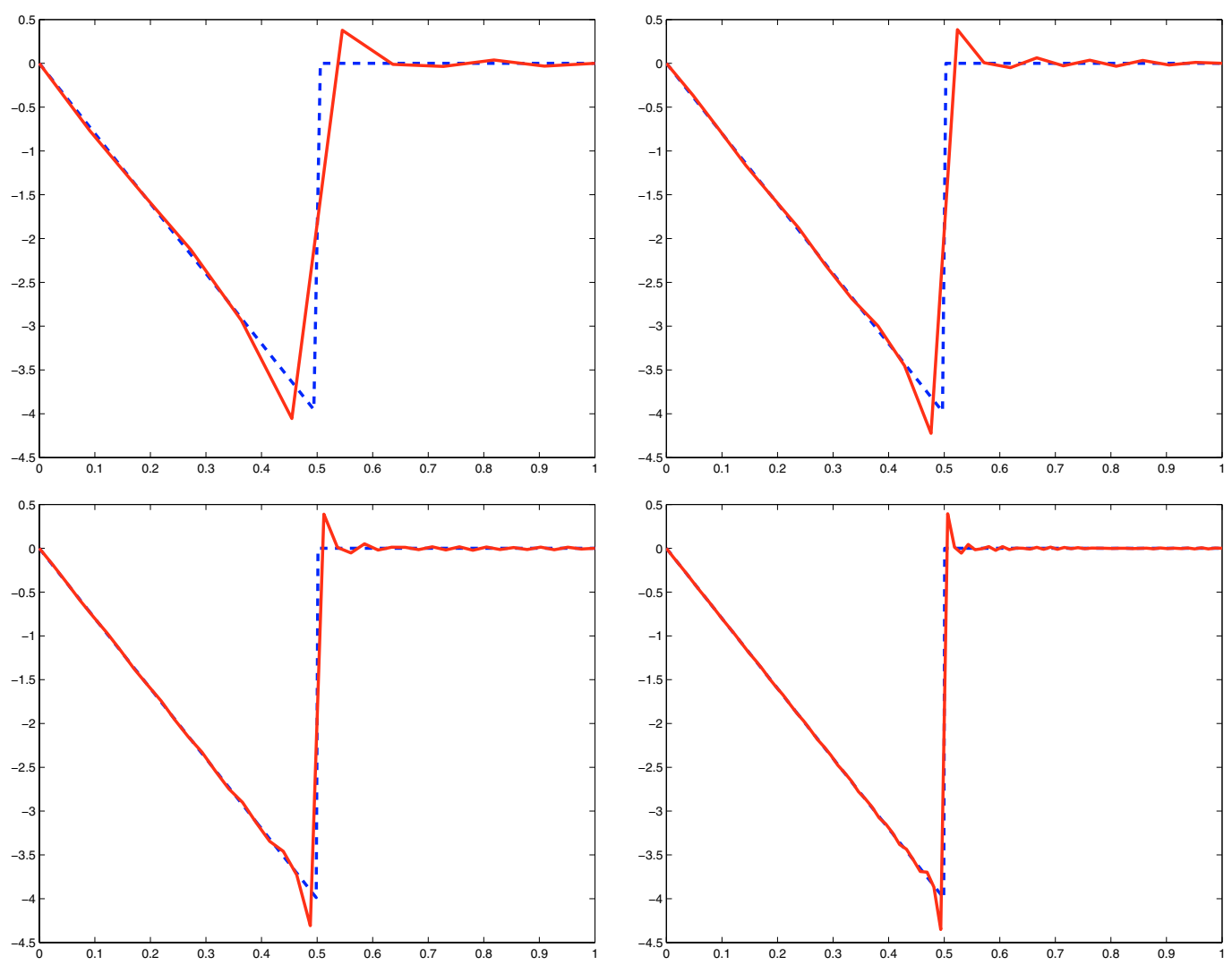

Figure 18. (Bi-Grid) $P\left(\hat{\boldsymbol{w}}_{1 \boldsymbol{h}}\right)(x)$ vs. $x \in[0,1]$ with the Bi-Grid method $-\nu=0.5, T=2.4$ and $h=1 / 21,1 / 41,1 / 81,1 / 161$.

$\left(\boldsymbol{y}_{\mathbf{0}}, \boldsymbol{y}_{\mathbf{1} \boldsymbol{h}}\right)$ are given by $(29)$, i.e.,

$$
\boldsymbol{y}_{\mathbf{0} \boldsymbol{h}}=\frac{1}{2} \sum_{k=1}^{J}\left[\bar{a}_{k, h}\left(1+c_{1 k}\right)+\frac{\bar{b}_{k, h}}{\mu_{k, h}} s_{1 k}\right] \boldsymbol{\phi}_{\boldsymbol{k}, \boldsymbol{h}}, \quad \boldsymbol{y}_{\mathbf{1} \boldsymbol{h}}=\sum_{k=1}^{J}\left[\bar{a}_{k, h} \frac{\left(c_{1 k}-1\right)}{\Delta t}+\frac{\bar{b}_{k, h}}{\mu_{k, h}} \frac{s_{1 k}}{\Delta t}\right] \boldsymbol{\phi}_{\boldsymbol{k}, \boldsymbol{h}} .
$$

We recall that the eigenvalues of $K$ are $\lambda_{k}^{K}=4 s_{k}^{2}, k=1, \ldots, J$. Therefore, using (154), for $(\theta, \alpha, \nu) \in \mathcal{C}$, the eigenvalues of $M_{0}^{\theta_{\nu}}$ and $M_{1}^{\theta_{\nu}}$ are

$$
\lambda_{k}^{M_{0}^{\theta_{\nu}}}=1-\left(1-\nu^{2}\right) s_{k}^{2}=c_{k}^{2}+\nu^{2} s_{k}^{2}, \quad \lambda_{k}^{M_{1}^{\theta_{\nu}}}=1-s_{k}^{2}=c_{k}^{2} .
$$

According to (83), this leads to

$$
\left(M_{0}^{\theta_{\nu}} \boldsymbol{y}_{\mathbf{1} \boldsymbol{h}}, K_{h}^{-1} M_{0}^{\theta_{\nu}} \boldsymbol{y}_{\mathbf{1} \boldsymbol{h}}\right)=\frac{1}{8} \sum_{k=1}^{J} \frac{\left(c_{k}^{2}+\nu^{2} s_{k}^{2}\right)^{2}}{s_{k}^{2}} h^{2}\left[\bar{a}_{k, h} \frac{\left(c_{1 k}-1\right)}{\Delta t}+\frac{\bar{b}_{k, h}}{\mu_{k, h}} \frac{s_{1 k}}{\Delta t}\right]^{2} .
$$

Similarly, we obtain

$$
\left(M_{0}^{\theta_{\nu}} \boldsymbol{y}_{\mathbf{o}},\left(M_{1}^{\theta_{\nu}}\right)^{-1} M_{0}^{\theta_{\nu}} \boldsymbol{y}_{\mathbf{0}}\right)=\frac{1}{8} \sum_{k=1}^{J} \frac{\left(c_{k}^{2}+\nu^{2} s_{k}^{2}\right)^{2}}{c_{k}^{2}}\left[\bar{a}_{k, h}\left(1+c_{1 k}\right)+\frac{\bar{b}_{k, h}}{\mu_{k, h}} s_{1 k}\right]^{2} .
$$


Finally, using $h^{2} / \Delta t^{2}=\nu^{-2}$, it follows that

$$
\begin{aligned}
\left\|\boldsymbol{y}_{\mathbf{0} \boldsymbol{h}}, \boldsymbol{y}_{\mathbf{1} \boldsymbol{h}}\right\|_{-1}^{2}= & \frac{1}{8} \sum_{k=1}^{J}\left(c_{k}^{2}+\nu^{2} s_{k}^{2}\right)^{2}\left[\bar{a}_{k, h}^{2}\left(\frac{\left(c_{1 k}-1\right)^{2}}{s_{k}^{2} \nu^{2}}+\frac{\left(c_{1 k}+1\right)^{2}}{c_{k}^{2}}\right)\right. \\
& \left.+\left(\frac{\bar{b}_{k, h}}{\mu_{k, h}}\right)^{2} s_{1 k}^{2}\left(\frac{1}{s_{k}^{2} \nu^{2}}+\frac{1}{c_{k}^{2}}\right)+\bar{a}_{k, h} \frac{\bar{b}_{k, h}}{\mu_{k, h}}\left(\frac{\left(c_{1 k}-1\right) s_{1 k}}{s_{k}^{2} \nu^{2}}+\frac{\left(c_{1 k}+1\right) s_{1 k}}{c_{k}^{2}}\right)\right] .
\end{aligned}
$$

Using (85) and (86), we have

Lemma 7.1.

$$
c_{1 k}=\frac{c_{k}^{2}-\nu^{2} s_{k}^{2}}{c_{k}^{2}+\nu^{2} s_{k}^{2}}, \quad s_{1 k}=\frac{2 c_{k} s_{k} \nu}{c_{k}^{2}+\nu^{2} s_{k}^{2}}, \quad 1+c_{1 k}=\frac{2 c_{k}^{2}}{c_{k}^{2}+\nu^{2} s_{k}^{2}}, \quad 1-c_{1 k}=\frac{2 s_{k}^{2} \nu^{2}}{c_{k}^{2}+\nu^{2} s_{k}^{2}} .
$$

Then, we obtain

and finally the result.

$$
\left\{\begin{array}{l}
\left(\frac{\left(c_{1 k}-1\right)^{2}}{s_{k}^{2} \nu^{2}}+\frac{\left(c_{1 k}+1\right)^{2}}{c_{k}^{2}}\right)=\left(\frac{1}{s_{k}^{2} \nu^{2}}+\frac{1}{c_{k}^{2}}\right)=\frac{4}{c_{k}^{2}+\nu^{2} s_{k}^{2}} \\
\left(\frac{\left(c_{1 k}-1\right) s_{1 k}}{s_{k}^{2} \nu^{2}}+\frac{\left(c_{1 k}+1\right) s_{1 k}}{c_{k}^{2}}\right)=0 .
\end{array}\right.
$$

\subsection{Appendix 2: Explicit computation of $\Lambda_{h, \Delta t}^{\theta, \alpha}$}

The aim of this second appendix is to compute explicitly the matrix associated to the HUM operator $\Lambda$ : $\left(H_{0}^{1}(0,1) \times L^{2}(0,1)\right) \rightarrow\left(H^{-1}(0,1) \times L^{2}(0,1)\right)$. In this respect, we use the following property:

$$
\left\langle\Lambda\left(w_{0}, w_{1}\right),\left(w_{0}, w_{1}\right)\right\rangle=\int_{0}^{1}\left(w_{x}(1, t)\right)^{2} \mathrm{~d} t, \quad \forall\left(w_{0}, w_{1}\right) \in\left(H_{0}^{1}(0,1) \times L^{2}(0,1)\right) .
$$

At the discrete level, $w_{x}(1, t)$ is approximated by $\frac{w_{J+2}^{n}-w_{J}^{n}}{2 h}=-\frac{w_{J}^{n}}{h}$, and $\Lambda$ by $\boldsymbol{\Lambda}_{\boldsymbol{h}, \boldsymbol{\theta} \boldsymbol{t}}^{\boldsymbol{\theta}, \boldsymbol{\alpha}} \in \mathcal{M}_{2 J \times 2 J}(\mathbb{R}) \operatorname{defined}$ as follows

$$
\left\langle\boldsymbol{\Lambda}_{\boldsymbol{h}, \boldsymbol{\Delta} \boldsymbol{t}}^{\boldsymbol{\theta}, \boldsymbol{\alpha}}\left(\boldsymbol{w}_{\mathbf{0} \boldsymbol{h}}, \boldsymbol{w}_{\mathbf{1} \boldsymbol{h}}\right),\left(\boldsymbol{w}_{\mathbf{0} \boldsymbol{h}}, \boldsymbol{w}_{\mathbf{1} \boldsymbol{h}}\right)\right\rangle=\Delta t \sum_{n=0}^{N-1}\left(\left|\frac{w_{J}^{n}}{h}\right|^{2}+\theta_{\nu}\left|\frac{w_{J}^{n+1}-w_{J}^{n}}{\Delta t}\right|^{2}\right),
$$

for all $\left(\boldsymbol{w}_{\mathbf{0} \boldsymbol{h}}, \boldsymbol{w}_{\mathbf{1} \boldsymbol{h}}\right) \in \mathbb{R}^{2 J}$, and $\boldsymbol{\phi}_{\boldsymbol{h}}^{\boldsymbol{n}} \in \mathbb{R}^{J}$ solution of

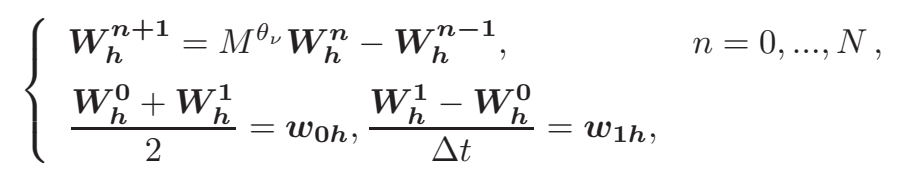

with $M^{\theta_{\nu}}=\left(M_{0}^{\theta_{\nu}}\right)^{-1}\left(2 M_{0}^{\theta_{\nu}}-\nu^{2} K\right) \in \mathcal{M}_{J \times J}(\mathbb{R})$.

A simple computation first leads to

Lemma 7.2. For $n=2, \ldots, N$ and $k=0, \ldots, n-1$, let us define the sequence $\left(a_{k}^{n}\right)$ as follows:

$$
\left\{\begin{array}{l}
a_{0}^{4 p}=a_{0}^{4 p+1}=1 ; a_{0}^{4 p+2}=a_{0}^{4 p+3}=-1 ; p>-1 \\
a_{p}^{p}=1, a_{p}^{p+1}=-1 ; p>1 \\
a_{k}^{n}=a_{k-1}^{n-1}-a_{k}^{n-2} ; n>k
\end{array} .\right.
$$


Then, for $n \geq 2$ even, we have

$$
\boldsymbol{W}_{\boldsymbol{h}}^{\boldsymbol{n}}=\sum_{k^{\text {even }} \in[0, n-2]} a_{k}^{n}\left(M^{\theta_{\nu}}\right)^{k} \boldsymbol{W}_{\boldsymbol{h}}^{\mathbf{0}}+\sum_{k^{\text {odd }} \in[1, n-1]} a_{k}^{n}\left(M^{\theta_{\nu}}\right)^{k} \boldsymbol{W}_{\boldsymbol{h}}^{\mathbf{1}},
$$

whereas for $n \geq 3$ odd, we have

$$
\boldsymbol{W}_{\boldsymbol{h}}^{\boldsymbol{n}}=\sum_{k^{\text {odd }} \in[1, n-2]} a_{k}^{n}\left(M^{\theta_{\nu}}\right)^{k} \boldsymbol{W}_{\boldsymbol{h}}^{\mathbf{0}}+\sum_{k^{\text {even }} \in[0, n-1]} a_{k}^{n}\left(M^{\theta_{\nu}}\right)^{k} \boldsymbol{W}_{\boldsymbol{h}}^{\mathbf{1}} .
$$

Definition 7.3. We define $E^{(n, q)}, O^{(n, q)} \in \mathcal{M}_{J \times J}(\mathbb{R})$ by

$$
E^{(n, q)}=\sum_{k^{\text {even }} \in[0, q]} a_{k}^{n}\left(M^{\theta_{\nu}}\right)^{k}, \quad O^{(n, q)}=\sum_{k^{\text {odd }} \in[1, q]} a_{k}^{n}\left(M^{\theta_{\nu}}\right)^{k} .
$$

From this definition, it results that

$$
\begin{array}{ll}
\boldsymbol{W}_{\boldsymbol{h}}^{\boldsymbol{n}}=E^{(n, n-2)} \boldsymbol{W}_{\boldsymbol{h}}^{\mathbf{0}}+O^{(n, n-1)} \boldsymbol{W}_{\boldsymbol{h}}^{\mathbf{1}}, & n \text { even, } \\
\boldsymbol{W}_{\boldsymbol{h}}^{\boldsymbol{n}}=O^{(n, n-2)} \boldsymbol{W}_{\boldsymbol{h}}^{\mathbf{0}}+E^{(n, n-1)} \boldsymbol{W}_{\boldsymbol{h}}^{\mathbf{1}}, & n \text { odd } .
\end{array}
$$

Lemma 7.4. For $n \geq 2$ even, we have

$$
\begin{aligned}
w_{J}^{n} w_{J}^{n}=\sum_{j, k=1}^{J}\left(E_{J j}^{(n, n-2)} P_{J k}^{(n, n-2)} w_{j}^{0} w_{k}^{0}+E_{J j}^{(n, n-2)} O_{J k}^{(n, n-1)} w_{j}^{0} w_{k}^{1}\right. & \\
& \left.+O_{J j}^{(n, n-1)} E_{J k}^{(n, n-2)} w_{j}^{1} w_{k}^{0}+O_{J j}^{(n, n-1)} O_{J k}^{(n, n-1)} w_{j}^{1} w_{k}^{1}\right)
\end{aligned}
$$

whereas for $n \geq 3$ odd, we have

$$
\begin{aligned}
w_{J}^{n} w_{J}^{n}=\sum_{j, k=1}^{J}\left(O_{J j}^{(n, n-2)} O_{J k}^{(n, n-2)} w_{j}^{0} w_{k}^{0}+O_{J j}^{(n, n-2)} E_{J k}^{(n, n-1)} w_{j}^{0} w_{k}^{1}\right. & \\
& \left.+E_{J j}^{(n, n-1)} O_{J k}^{(n, n-2)} w_{j}^{1} \phi_{k}^{0}+E_{J j}^{(n, n-1)} E_{J k}^{(n, n-1)} w_{j}^{1} w_{k}^{1}\right) .
\end{aligned}
$$

We add that

$$
w_{J}^{0} w_{J}^{0}=\sum_{j, k=1}^{J} \delta_{J j} \delta_{J k} w_{j}^{0} w_{k}^{0}, \quad w_{J}^{1} \phi_{J}^{1}=\sum_{j, k=1}^{J} \delta_{J j} \delta_{J k} w_{j}^{1} w_{k}^{1} .
$$

In order to simplify the previous expression, we introduce the following notation:

Definition 7.5. For $n \geq 2$ even, we note

$$
\begin{array}{ll}
A_{j k, J}^{0,0, n}=E_{J j}^{(n, n-2)} E_{J k}^{(n, n-2)}, & A_{j k, J}^{0,1, n}=E_{J j}^{(n, n-2)} O_{J k}^{(n, n-1)} \\
A_{j k, J}^{1,0, n}=O_{J j}^{(n, n-1)} E_{J k}^{(n, n-2)}, & A_{j k, J}^{1,1, n}=O_{J j}^{(n, n-1)} O_{J k}^{(n, n-1)} .
\end{array}
$$

For $n \geq 3$, we note

$$
\begin{array}{ll}
A_{j k, J}^{0,0, n}=O_{J j}^{(n, n-2)} O_{J k}^{(n, n-2)}, & A_{j k, J}^{0,1, n}=O_{J j}^{(n, n-2)} E_{J k}^{(n, n-1)} \\
A_{j k, J}^{1,0, n}=E_{J j}^{(n, n-1)} O_{J k}^{(n, n-2)}, & A_{j k, J}^{1,1, n}=E_{J j}^{(n, n-1)} E_{J k}^{(n, n-1)}
\end{array}
$$


We add

$$
\begin{aligned}
& A_{j k, J}^{0,0,0}=\delta_{J j} \delta_{J k} ; A_{j k, J}^{0,1,0}=A_{j k, J}^{1,0,0}=A_{j k, J}^{1,1,0}=0 ; \\
& A_{j k, J}^{0,0,1}=A_{j k, J}^{0,1,1}=A_{j k, J}^{1,0,1}=0 ; A_{j k, J}^{1,1,1}=\delta_{J j} \delta_{J k} .
\end{aligned}
$$

Therefore, from Lemma 7.4 and Definition 7.5, it follows that

$$
\begin{aligned}
\Delta t \sum_{n=0}^{N-1}\left|\frac{w_{J}^{n}}{h}\right|^{2} & =\frac{\Delta t}{h^{2}} \sum_{n=0}^{N-1} \sum_{j, k=1}^{J}\left(A_{j k, J}^{0,0, n} w_{j}^{0} w_{k}^{0}+A_{j k, J}^{0,1, n} w_{j}^{0} w_{k}^{1}+A_{j k, J}^{1,0, n} w_{j}^{1} w_{k}^{0}+A_{j k, J}^{1,1, n} w_{j}^{1} w_{k}^{1}\right) \\
& =\left\langle\overline{\boldsymbol{\Lambda}}_{\boldsymbol{h}, \boldsymbol{\theta} \boldsymbol{\theta}, \mathbf{1}}\left(\boldsymbol{w}_{\boldsymbol{h}}^{\mathbf{0}}, \boldsymbol{w}_{\boldsymbol{h}}^{\mathbf{1}}\right),\left(\boldsymbol{w}_{\boldsymbol{h}}^{\mathbf{0}}, \boldsymbol{w}_{\boldsymbol{h}}^{\mathbf{1}}\right)\right\rangle,
\end{aligned}
$$

with

and

$$
\overline{\boldsymbol{\Lambda}}_{\boldsymbol{h}, \boldsymbol{\Delta} \boldsymbol{\theta}, \mathbf{1}}^{\boldsymbol{\theta}}=\left(\begin{array}{cc}
A_{11} & A_{12} \\
A_{12}^{T} & A_{22}
\end{array}\right)_{2 J \times 2 J}, \quad A_{11}, A_{12}, A_{22} \in \mathcal{M}_{J \times J}(\mathbb{R})
$$

$$
\left(A_{11}\right)_{k j}=\frac{\Delta t}{h^{2}} \sum_{n=0}^{N-1} A_{j k, J}^{0,0, n}, \quad\left(A_{12}\right)_{k j}=\frac{\Delta t}{h^{2}} \sum_{n=0}^{N-1} A_{j k, J}^{1,0, n}, \quad\left(A_{22}\right)_{k j}=\frac{\Delta t}{h^{2}} \sum_{n=0}^{N-1} A_{j k, J}^{1,1, n} .
$$

Besides, from $\phi_{h}^{0}=e_{0 h}-\Delta t / 2 e_{1 h}, \phi_{h}^{1}=e_{0 h}+\Delta t / 2 e_{1 h}$, we deduce that

$$
\left\langle\bar{\Lambda}_{h, \Delta t, 1}^{\theta, \alpha}\left(w_{h}^{0}, w_{h}^{1}\right),\left(w_{h}^{0}, w_{h}^{1}\right)\right\rangle=\left\langle\Lambda_{h, \Delta t, 1}^{\theta, \alpha}\left(w_{0 h}, w_{1 h}\right),\left(w_{0 h}, w_{1 h}\right)\right\rangle
$$

with

$$
\boldsymbol{\Lambda}_{\boldsymbol{h}, \boldsymbol{\Delta} \boldsymbol{t}, \mathbf{1}}^{\boldsymbol{\theta}, \boldsymbol{1}}=\left(\begin{array}{cc}
A_{11}+A_{12}+A_{12}^{T}+A_{22} & -\frac{\Delta t}{2}\left(A_{11}-A_{12}+A_{12}^{T}-A_{22}\right) \\
-\frac{\Delta t}{2}\left(A_{11}+A_{12}-A_{12}^{T}-A_{22}\right) & \left(\frac{\Delta t}{2}\right)^{2}\left(A_{11}-A_{12}-A_{12}^{T}+A_{22}\right)
\end{array}\right)_{2 J \times 2 J} .
$$

We verify that $\Lambda_{\boldsymbol{h}, \boldsymbol{\Delta} \boldsymbol{t}, \mathbf{1}}^{\boldsymbol{\theta}, \boldsymbol{\alpha}}$ is a symmetric matrix, thanks to the symmetry of $A_{11}$ and $A_{22}$.

Finally, performing similar computations with the term in (178) factor of $\theta_{\nu}$, we obtain an explicit expression of the matrix $\Lambda_{h, \Delta t}^{\theta, \alpha}$

Acknowledgements. Professors Carlos Castro, Sorin Micu and Enrique Zuazua are gratefully acknowledged for fruitful discussions during the post-doctoral stay of the author at the Department of Mathematics of the "Universidad Autónoma de Madrid".

\section{REFERENCES}

[1] M. Asch and G. Lebeau, Geometrical aspects of exact boundary controllability for the wave equation: a numerical study. COCV 3 (1998) 163-212.

[2] H.T. Banks, K. Ito and Y. Wang, Exponentially stable approximations of weakly damped wave equations. Ser. Num. Math., Birkhäuser 100 (1990) 1-33.

[3] F. Bourquin, Numerical methods for the control of flexible structures. J. Struct. Control 8 (2001).

[4] C. Castro and S. Micu, Boundary controllability of a semi-discrete linear 1-D wave equation with mixed finite elements. SIAM J. Numer. Anal., submitted.

[5] C. Castro, S. Micu and A. Münch, Boundary controllability of a semi-discrete linear 2-D wave equation with mixed finite elements, submitted.

[6] I. Charpentier and Y. Maday, Identification numérique de contrôles distribués pour l'équation des ondes. C.R. Acad. Sci. Paris Sér. I 322 (1996) 779-784.

[7] G.C. Cohen, Higher-order Numerical Methods for Transient Wave Equations. Scientific Computation, Springer (2002).

[8] R. Glowinski, Ensuring well-posedness by analogy: Stokes problem and boundary control for the wave equation. J. Comput. Phys. 103 (1992) 189-221.

[9] R. Glowinski, C.H. Li and J.-L. Lions, A numerical approach to the exact boundary controllability of the wave equation (I). Dirichlet Controls: Description of the numerical methods. Japan J. Appl. Math. 7 (1990) 1-76. 
[10] R. Glowinski, W. Kinton and M.F. Wheeler, A mixed finite element formulation for the boundary controllability of the wave equation. Int. J. Numer. Methods Engrg. 27 (1989) 623-636.

[11] G.H. Golub and C. Van Loan, Matrix Computations. Johns Hopkins Press, Baltimore (1989).

[12] J.A. Infante and E. Zuazua, Boundary observability for the space-discretizations of the 1-D wave equation. ESAIM: M2AN 33 (1999) 407-438.

[13] A.E. Ingham, Some trigonometrical inequalities with applications to the theory of series. Math. Z. 41 (1936) 367-369.

[14] V. Komornik, Exact controllability and Stabilization - The multiplier method. J. Wiley and Masson (1994).

[15] S. Krenk, Dispersion-corrected explicit integration of the wave equation. Comp. Methods Appl. Mech. Engrg. 191 (2001) 975-987.

[16] J.L. Lions, Contrôlabilité exacte - Pertubations et stabilisation de systèmes distribués, Tome 1, Masson, Paris (1988).

[17] S. Micu, Uniform boundary controllability of a semi-discrete 1-D wave equation. Numer. Math. 91 (2002) 723-728.

[18] A. Münch, Family of implicit schemes uniformly controllable for the 1-D wave equation. C.R. Acad. Sci. Paris Sér. I 339 (2004) 733-738.

[19] A. Münch and A.F. Pazoto, Uniform stabilization of a numerical approximation of a locally damped wave equation. ESAIM: $C O C V$, submitted.

[20] M. Negreanu and E. Zuazua, Uniform boundary controllability of a discrete 1-D wave equation. Systems Control Lett. 48 (2003) 261-280.

[21] M. Negreanu and E. Zuazua, Discrete Ingham inequalities and applications. C.R. Acad. Sci. Paris Sér. I 338 (2004) $281-286$.

[22] M. Negreanu and E. Zuazua, Convergence of a multi-grid method for the controlabillity of the 1-D wave equation. C.R. Acad. Sci. Paris, Sér. I 338 (2004) 413-418.

[23] P.A. Raviart and J.M. Thomas, Introduction à l'analyse numérique des équations aux dérivées partielles. Masson, Paris (1983).

[24] D.L. Russell, Controllability and stabilization theory for linear partial differential equations: recent progress and open questions. SIAM Rev. 20 (1978) 639-737.

[25] J.M. Urquiza, Contrôle d'équations des ondes linéaires et quasilinéaires. Ph.D. Thesis Université de Paris VI (2000).

[26] E. Zuazua, Boundary observability for finite-difference space semi-discretizations of the 2-D wave equation in the square. $J$. Math. Pures. Appl. 78 (1999) 523-563.

To access this journal online: www.edpsciences.org 